\title{
UNDISCOVERED MINERAL RESOURCES OF SOUTHEASTERN ALASKA-- REVISED MINERAL-RESOURCE-ASSESSMENT-TRACT DESCRIPTIONS
}

By

\author{
David A. Brew 1 \\ Donald J. Grybeck ${ }^{2}$ \\ Cliff D. Taylor ${ }^{3}$ \\ Robert C. Jachens 1 \\ Dennis P. Cox 1 \\ David F. Barnes 1 \\ Richard D. Koch ${ }^{1}$ \\ Robert L. Morin 1 \\ and \\ James L. Drinkwater 1
}

U.S. Geological Survey Open-File Report 96-716

This report is preliminary and has not been edited or reviewed for conformity with U.S. Geological Survey editorial standards or with the North American Stratigraphic Code. Any use of trade, firm, or product names is for descriptive purposes only and does not imply endorsement by the U.S. Government

1 U.S. Geological Survey

Menlo Park, CA 94025-3591, U.S.A.

2U.S. Geological Survey

Anchorage, AK 99508-4667

${ }^{3}$ U.S. Geological Survey

Denver, CO 80225 


\section{UNDISCOVERED MINERAL RESOURCES OF SOUTHEASTERN ALASKA-- REVISED MINERAL-RESOURCE-ASSESSMENT-TRACT DESCRIPTIONS}

By

David A. Brew ${ }^{1}$, Donald J. Grybeck ${ }^{2}$, Cliff D. Taylor ${ }^{3}$, Robert C. Jachens ${ }^{1}$, Dennis P. Cox ${ }^{1}$, David F. Barnes ${ }^{1}$, Richard D. Koch ${ }^{1}$, Robert L. Morin ${ }^{1}$, and James L. Drinkwater ${ }^{1}$

1U.S. Geological Survey, Menlo Park, CA 94025-3591, 2U.S. Geological Survey, Anchorage, AK 995064667, 3U.S. Geological Survey, Denver, CO 80225

\section{INTRODUCTION}

This report presents revisions, re-descriptions, and additions to the mineral-resource-assessment-tract information contained in the Tongass National Forest and adjacent areas reports by Brew and others (1991) and Brew and Drinkwater (1991).The changes to some individual tracts are significant, but the overall effect of the changes on the conclusions of those two reports is not that great. This is because the changes are confined to a small proportion of the tracts present in southeastern Alaska. The reexamination and revision was done by the present authors together as a team; it relied especially on the interpretations of additional geochemical data by C.D. Taylor and of additional geophysical data by R.C. Jachens.

The reason for this reexamination and additional interpretation was the national-scale assessment of undiscovered mineral resources undertaken by the Office of Mineral Resources, Geologic Division, U.S. Geological Survey in 1993 (D.P. Cox and S.D. Ludington, written commun., 1993). As part of that national project, the State of Alaska was divided into subregions, of which southeastern Alaska is one, and the undiscovered mineral resources of each subregion were assessed. As just noted, the required assessment of southeastern Alaska followed the earlier comprehensive assessment by Brew and others (1991) and Brew and Drinkwater (1991), which had resulted in the delineation of many separate tracts and the estimation of the numbers of undiscovered deposits of different types in those tracts. Because of this prior assessment, the subsequent one involved mainly reexamination of the previous material, but some additional geochemical and geophysical data were incorporated also. However, with the exception of both the newly defined and the significantly revised tracts noted below, the estimated numbers of deposits within each tract are taken directly from Brew and others (1991).

The information developed for the separate tracts during the reexamination process was contributed to the Alaska statewide assessment, but, because of the greater detail of the southeastern Alaska assessment in comparison to the rest of Alaska, the individual southeastern Alaska tracts were amalgamated into a much smaller number for the purpose of the statewide assessment (T.D. Light, U.S. Geological Survey, written commun., 1995). This present report, then, not only documents the changes made in our detailed tract analysis and thus provides additional information for the users of Brew and others (1991) and Brew and Drinkwater (1991) but it also provides the background information for the tracts that have been amalgamated in the statewide assessment.

The remainder of this report has three main parts: the first follows immediately and consists of noting which tracts were changed and how; the second consists of copies of the individual pages of the FilemakerProß tract file, and the third is the 1:1,000,000-scale map (plate 1) showing all the assessment tract boundaries, including the changed ones.

The definitions and background information given in Brew and others (1991, especially p. 16-17) apply to this report and are not repeated here. Please note that where a tract has more than one part that are geographically separated, the letters "a" and " $\mathrm{A}$ " are used interchangeably on the map and in the tract descriptions. Note also that " $T$ " is the abbreviation for English tons and "mt" is that for metric tonnes. 
Listed in the next paragraph are the significant changes that alter the information in Brew and others (1991). All other items in that prior report, including the catalog of deposits (Brew and others, 1991, table 2), remain the same for the present time. Obviously, the estimated metal content and its inferred value (Brew and others, 1991 , tables 5,7-12) would change for the items noted below, were they to be recalculated.

Tracts 28CR, 09PA, and 10PA are new and are based on new geophysical data and reexamination of geochemical information. Tract $13 \mathrm{SI}$ was split into two tracts based on new field mapping and the reexamination of geochemical data, with the northern one being $09 \mathrm{JU}$ and the southern one being $13 \mathrm{SI}$. Boundary changes were made to tracts $05 \mathrm{JU}, 08 \mathrm{KC}, 12 \mathrm{PE}$, and $16 \mathrm{SK}$ based mainly on reexamination of field mapping information. Some model assignments were changed for tracts 10DE (Nokleberg and others, 1995, p. 115), 03JU, 09CR, 26CR; and 14SI in order to better agree with mineral-deposit models (Cox and Singer, 1986); and all model 17 (porphyry $\mathrm{Cu}$ ) assignments were changed to the more recently described model 17.1 (British-Columbia-Alaska porphyry Cu; Menzie and Singer, 1993). The reader is referred to the tract descriptions below and those in Brew and others (1991, tables 4 and 5) to make more detailed comparisons.

\section{TRACT DESCRIPTIONS}

The FilemakerPro ${ }^{\circledR}$ tract file contains the essential information for each tract, using a format designed by R.D. Koch and revised by D.J. Grybeck. Each of the 120-plus mineral-resource-assessment tracts in southeastern Alaska is represented by a single page. The information on that page is explained as follows.

Southeastern Alaska: The region containing the tract

Tract Name:

Based on the general location of the tract

Tract No:

The map (plate 1) and tract-sheet numeroalphic designator; the origin of these designators is explained in Brew and others (1991)

Geology:

Brief description of the rocks in the tract used in defining the tract boundaries

Geochemistry:

Brief description of the geochemical anomalies, if any, used in defining the tract boundaries

Geophysics:

Brief description of the geophysical anomalies, if any, used in defining the tract boundaries

Significant Deposits: (w/ Production and

Reserves)

The alphanumeric designators for specific known mineral deposits as described in table 2 of Brew and others (1991); production and reserve information is included for each deposit for which it is available

Undiscovered Deposit Type: The mineral deposit types expected to be present in the tract are listed, first by their number, then by name, following the descriptive models of Cox and Singer (1986) and Menzie and Singer (1993); other models are briefly described by Nokleberg and others (1988)

Number of Undiscovered Deposits at Probability Percentile 95, 90, 50, 10, 05: Estimates of the maximum number of deposits judged to be present in the tract, using the grade and tonnage models of Cox and Singer (1986) and of Menzie and Singer (1993) for the BC-AK porphyry copper deposits (model 17.1) and a basaltic copper deposit model based on the data of White (1978)

Grade/Tonnage Model?: Availabilty as yes or no, based on the data in Cox and Singer (1986) and in Menzie and Singer (1993)

Tract $\left(\mathrm{km}^{2}\right)$ : 


\section{TRACT DESCRIPTIONS--Continued}

Comments:

Discussion:
General charaterization of geologic and geochemical mapping, terrain, vegetation, access, and land ownership

Other points, if any

\section{REFERENCES CITED}

Brew, D.A., Drew, L.J., Schmidt, J.M., Root, D.H., and Huber,'D.F., 1991, Undiscovered locatable mineral resources of the Tongass National Forest and adjacent lands, southeastern Alaska: U.S. Geological Survey Open-File Report 91-10, 11 fig., 16 pl. 1:250,000 and 1:500,000-scale, 370 p.

Brew, D.A., and Drinkwater, J.L., 1991, Tongass Timber Reform Act Wilderness Areas supplement to U.S. Geological Survey Open-File Report 91-10 (Undiscovered locatable mineral resources of the Tongass National Forest and adjacent lands, southeastern Alaska): U.S. Geological Survey OpenFile Report 91-343: 56 p.

Cox, D.P. and Singer, D.A., 1986, Mineral deposit models: U.S. Geological Survey Bulletin 1693, 379 p.

Menzie, W.D. and Singer, D.A.,1993, Grade and tonnage model of porphyry $\mathrm{Cu}$ deposits in British Columbia, Canada, and Alaska, U.S.A.: U.S. Geological Survey Open-File report 93-275, 9 p.

Nokleberg, W.J., Bundtzen, T.K., Brew, D.A., Grybeck, D., Robinson, M.S., Smith, T.E., and Yeend, W., 1988, Metallogeny and major mineral deposits of Alaska: U.S. Geological Survey Open-File Report 88-73, 2 pl., 97 p.

Nokleberg, W.J., Bundtzen, T.K., Brew, D.A., and Plafker, G., 1995, Metallogenesis and tectonics of porphyry $\mathrm{Cu}$ and $\mathrm{Mo}(\mathrm{Au}, \mathrm{Ag}$ ) and granitoid-hosted $\mathrm{Au}$ deposits of Alaska, in Schroeter, T.G., ed., Porphyry deposits of the northwestern Cordillera-A sequel to CIM Special Volume 15, Canadian Institute of Mining, Metallurgy, and Petroleum Special Volume 46, p. 103-141.

Thomson, L., 1996, Mine [Greens Creek] invests $\$ 80$ million to reopen: Juneau Empire, March 7, 1996, p. 1,10 .

White, W.S., 1968, The native copper deposits of northern Michigan, in Ridge, J.D., ed., Ore deposits of the United States, 1933-1967 (The Graton-Sales Volume): American Institute of Mining Metallurgical, and Petroleum Engineers, Inc., v. 1, p. 303-326. 
Geology: Paleozoic and Late Proterozoic clastic carbonate and volcanic rocks are intruded and metamorphosed by Tertiary plutons.

\section{Geochemistry:}

Geophysics:

Significant Deposits: None

(w/Production and

Reserves)

\section{Undiscovered Deposit Type}

Number of Undiscovered Deposits

1) $14 a$ W skarn

$\begin{array}{ccccc}95 & 90 & 50 & 10 & 05 \\ 0 & 0 & 0 & 0 & 1 \\ 0 & 0 & 0 & 0 & 1 \\ 0 & 0 & 0 & 0 & 1\end{array}$

Model?

2) $18 b$ Cu skarn

3) $18 \mathrm{c} \mathrm{Zn-Pb}$ skarn

Tract $\left(\mathrm{km}^{2}\right): \quad 32$

Comments: Reconnaissance geologic mapping and geochemical sampling by USGS; essentially unprospected. Small tract. Remote, steep, extensive glacier cover. $100 \%$ in Tongass National Forest.

Discussion: Includes USGS OFR 91-010 tracts 01(B)AL and 12JU. 
Tract Name: Chilkoot Range ultramafic bodies (A, B, C)

Iract No: O2AL

Geology: Alpine-type peridotite bodies associated with gabbro, amphibolite, and greenstone intruded by Tertiary granitic bodies.

\section{Geochemistry:}

Geophysics: There may be aeromagnetic anomalies associated with these bodies.

\section{Significant Deposits: None}

(w/Production and

Reserves)

\section{Undiscovered Deposit Type}

1) 8a Podiform chromite (minor)

\section{Number of Undiscovered Deposits at Probabillity Percentile}

$95 \quad 90 \quad 50 \quad 10 \quad 05$

\begin{tabular}{ll|lll}
0 & 0 & 0 & 0 & 1
\end{tabular}

GraderTonnage

Model?

Yes

$\operatorname{Tract}\left(\mathrm{km}^{2}\right): \quad 18$

Comments: Reconnaissance geologic mapping and geochemical sampling by USGS; essentially unprospected. Small tract. Remote, steep, extensive glacier cover. Good exposures. $100 \%$ in Tongass National Forest.

Discussion: 
Geology: Low-metamorphic-grade metabasalt flows and tuffs of Triassic age associated with clastic and carbonate rocks. Minor supergene Cu minerals present locally.

\section{Geochemistry:}

\section{Geophysics:}

Significant Deposits: None

(w/Production and

Reserves)

Undiscovered Deposit Type

1) 24a Cyprus massive sulfide

\section{Number of Undiscovered Deposits} at Probability Percentile

$\begin{array}{lllll}95 & 90 & 50 & 10 & 05\end{array}$

$0 \quad 0 \quad 0 \quad 0 \quad 1$

Grade/Tonnage Model?

Yes

$\operatorname{Tract}\left(\mathrm{km}^{2}\right): 21$

Comments: Reconnaissance geologic mapping and geochemical sampling by USGS; essentially unprospected. Small tract. Remote, steep, extensive glacier cover. Good exposures. $100 \%$ in Tongass National Forest.

Discussion: 
Tract Name: Bradfield Canal Coast Mountains: (A) Elbow Mountain, (B)

Mount Whipple, (C) Craig River, (D) Mount Lewis Cass

Iract No: 01BC

Geology: Vein, skarn, and disseminated sulfide deposits occur in schist, gneiss, and marble of original Late Proterozoic(?) to Mesozoic age intruded by Tertiary plutons.

Geochemistry: Stream-sediment and bedrock anomalies: $\mathrm{Cu}, \mathrm{Pb}, \mathrm{Zn}, \mathrm{Ag}, \mathrm{Mo}, \mathrm{Au}$.

Geophysics:

Significant Deposits; (A) None; (B) None; (C) BC006; (D) BC005: 517,000 mt inferred w/ 0.30\% Cu, 55.0\% (w/Production and Fe (Coldwell, 1990), BC009

Reserves)

\section{Undiscovered Deposit Type}

1) 22c Polymetallic vein

2) $18 \mathrm{c} \mathrm{Zn}-\mathrm{Pb}$ skarn

3) 18d Fe skam

4) Porphyry Cu-Mo

Number of Undiscovered Deposits at Probability Percentile

$\begin{array}{lllll}95 & 90 & 50 & 10 & 05\end{array}$

$\begin{array}{lllll}0 & 0 & 0 & 0 & 1\end{array}$

$\begin{array}{lllll}0 & 0 & 0 & 0 & 1\end{array}$

$\begin{array}{llllll}0 & 0 & 0 & 1 & 2\end{array}$

$\begin{array}{lllll}0 & 0 & 0 & 0 & 1\end{array}$

GraderTonnage Model?

\begin{tabular}{|c|}
\hline Yes \\
\hline Yes \\
\hline Yes \\
\hline Yes \\
\hline
\end{tabular}

Tract $\left(\mathrm{km}^{2}\right): 212$ total: (A) 14, (B) $28,(C) 52$, and (D) 118

Comments: Reconnaissance geologic mapping and geochemical sampling by USGS. Remote and rugged; significant glacier and permanent snow cover. $7 \%$ in USFS Stikine-LeConte Wilderness, $82 \%$ in USFS Tongass National Forest, $11 \%$ in USFA Misty Fiords Wilderness.

Discussion: Fe-skarn resources in subtract (D) only. Close to active exploration areas in Jurassic volcanic rocks and plutons in nearby British Columbia. 
Geology: Leucocratic biotite-bearing alkalic granite stock in NE part of tract; quartz-porphyry thyolitic dikes in SW part.

Geochemistry: Stream-sediment anomalies: $\mathrm{Pb}, \mathrm{Y}, \mathrm{Be}, \mathrm{Nb}, \mathrm{Sn}, \mathrm{Mo}, \mathrm{Zn}, \mathrm{Ag}, \mathrm{Cu}$

Geophysics: Aeroradioactivity anomaly.

Significant Deposits: $\mathrm{BCOO4}$

(w/Production and

Reserves)

\section{Undiscovered Deposit Type}

1) 11d Th-RE veins (AP/THRE)

2) Polymetallic vein

3) 21b Porphyry Mo (low F)

\section{Number of Undiscovered Deposits at Probability Percentile}

$\begin{array}{lllll}95 & 90 & 50 & 10 & 05\end{array}$

$\begin{array}{lllll}- & - & - & - & - \\ 0 & 0 & 0 & 0 & 1 \\ 0 & 0 & 0 & 0 & 1\end{array}$

\section{Grade/Tonnage}

Model?

Tract $\left(\mathrm{km}^{2}\right): \quad 219$

Comments: Reconnaissance geologic mapping and geochemical sampling by USGS. Moderate amount of private prospecting and some exploration drilling. Remote, rugged, some thick brush and timber locally. $100 \%$ in USFS Tongass National Forest.

Discussion: Moderately well explored. 
Geology: Deformed and metamorphosed Late Proterozoic(?) to Mesozoic clastic and volcanic rocks are intruded by Late Cretaceous tonalite and granodiorite and by the latest Cretaceous to Paleocene Great tonalite sill; vein deposits present.

Geochemistry: Stream-sediment anomalies: $\mathrm{Zn}, \mathrm{Cu}, \mathrm{Mo}, \mathrm{Ag}, \mathrm{Pb}, \mathrm{Sn}$. Bedrock anomalies: $\mathrm{Zn}, \mathrm{Cu}, \mathrm{Pb}$, Mo, Ag, Au.

Geophysics:

Signlficant Deposits: BC002, 003; PE038, 041, 042

(w/Production and

Reserves)

Number of Undiscovered Deposits

\section{Undiscovered Deposit Type}

1) $28 \mathrm{c}$ Sierran kuroko massive sulfide

2) 22c Polymetallic vein at Probability Percentile

$95 \quad 90 \quad 50 \quad 10 \quad 05$

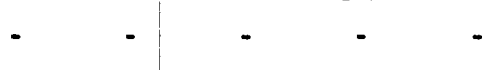

0
1

2
Grade/Tonnage Model?

Yes

Tract $\left(\mathrm{km}^{2}\right): \quad 499$ total: 425 in $\mathrm{BC}$ and 74 in PE

Comments: Reconnaissance geologic mapping and geochemical sampling by USGS. Moderate amount of private prospecting in North part of tract, less to South. In part close to tidewater, in part remote and rugged; some thick brush and timber locally. $91 \%$ in USFS Tongass National Forest; $9 \%$ in USFS Stikine-LeConte Wilderness.

Discussion: Includes USGS OFR 91-010 tracts 19PE and 04BC. 


\section{Southeastern Alaska}

Tract Name: Harding River

Iract No: $05 \mathrm{BC}$

Geology: Deformed and metamorphosed schist, gneiss, and minor marble derived from Late Proterozoic(?) to Mesozoic clastic and volcanic rocks are intruded by Early Tertiary granodionite plutons on the northeast and by the latest Cretaceous to Paleocene Great tonalite sill on the southwest

Geochemistry: Bedrock anomalies: $\mathrm{Mo}, \mathrm{Ag}, \mathrm{Cu}, \mathrm{Pb}, \mathrm{Zn}$.

\section{Geophysics:}

Significant Deposits: None

(w/Production and

Reserves)

\section{Undiscovered Deposit Type}

1) $22 c$ Polymetallic vein

2) Zn-Pb skam

\section{Number of Undiscovered Deposits at Probability Percentile}

$\begin{array}{ccccc}95 & 90 & 50 & 10 & 05 \\ 0 & 0 & 0 & 0 & 1\end{array}$

No est. No est. No est. No est. No est.

\section{GradefTonnage}

Model?

Yes

Tract $\left(\mathrm{km}^{2}\right): \quad 142$

Comments: Reconnaissance geologic mapping and geochemical sampling by USGS. Small amount of private prospecting. In part close to tidewater, in part remote, rugged, steep; very extensive glacier cover locally. $100 \%$ in USFS Tongass National Forest.

Discussion: 
Tract Name: Mount Stoeckl

Iract No: 06BC

Geology: Eocene porphyritic quartz monzonite intrudes schist, gneiss, and marble of Late Proterozoic(?) to Mesozoic age.

Geochemistry: Bedrock anomalies: U.

Geophysics:

Significant Deposits: BC010

(w/Production and

Reserves)

\section{Number of Undiscovered Deposits}

\section{at Probability Percentile}

Undiscovered Deposit Type

95

$90 \quad 50 \quad 10 \quad 05$

GraderTonnage

Model?

1) Felsic plutonic $U$

2) Th-RE veins

Tract $\left(\mathrm{km}^{2}\right): \quad 39$

Comments: Reconnaissance geologic mapping and geochemical sampling by USGS. Small amount of private prospecting. Small tract. Remote, rugged, steep; much glacier and permanent snow cover. $100 \%$ in USFS Misty Fiords Wilderness.

Discussion: 
Geology: Schist, gneiss, and minor marble of Late Proterozoic(?) to Mesozoic age.

Geochemistry: Bedrock anomalies: $\mathrm{Cu}, \mathrm{Ag}, \mathrm{Pb}, \mathrm{Zn}, \mathrm{Mo}, \mathrm{Sn}$.

Geophysics:

Significant Deposits: BC008

(w/Production and

Reserves)

Undiscovered Deposit Type

1) 18b Cu skam

2) 18d Fe skam
Number of Undiscovered Deposits at Probability Percentile

$\begin{array}{lllll}95 & 90 & 50 & 10 & 05\end{array}$

$\begin{array}{lllll}0 & 0 & 0 & 0 & 1\end{array}$

$0 \quad 0 \quad 0 \quad 0 \quad 1$

Grade/Tonnage Model?

\begin{tabular}{|c|}
\hline Yes \\
\hline Yes \\
\hline
\end{tabular}

Tract $\left(\mathrm{km}^{2}\right): 76$

Comments: Reconnaissance geologic mapping and geochemical sampling by USGS. Small amount of private prospecting. Not far from tidewater, rugged; heavy timber and brush locally. $100 \%$ in USFS Misty Fiords Wilderness.

\section{Discussion:}


Tract Name: Gracey Creek Glacier

Iract No: 08BC

Geology: Schist, gneiss, and minor marble of Late Proterozoic(?) to Mesozoic age.

Geochemistry: Bedrock anomalies: $\mathrm{Cu}, \mathrm{Zn}, \mathrm{Pb}, \mathrm{Ag}$.

Geophysics:

Significant Deposits: BC011

(w/Production and

Reserves)

Undiscovered Deposit Type

1) 22c Polymetallic vein
Number of Undiscovered Deposits

at Probability Percentile

$\begin{array}{lllll}95 & 90 & 50 & 10 & 05\end{array}$

$\begin{array}{lllll}0 & 0 & 0 & 0 & 1\end{array}$

\section{Grade/Tonnage}

Model?

Yes

$\operatorname{Tract}\left(\mathrm{km}^{2}\right): \quad 60$

Comments: Reconnaissance geologic mapping and geochemical sampling by USGS. Remote; rugged; extensive glacier and permanent snow cover. $100 \%$ in USFS Misty Fiords Wilderness.

Discussion: 


\section{Southeastern Alagka}

Tract Name: Burroughs Bay

Iract No: $10 \mathrm{BC}$

Geology: Quartz-porphyry dikes associated with molybdenite-bearing K-spar porphyritic biotite granite and quartz monzonite of Miocene age.

Geochemistry: Bedrock and stream-sediment anomalies: $\mathrm{Mo}, \mathrm{Cu}, \mathrm{Pb}, \mathrm{Zn}$.

Geophysics:

Significant Deposits; KCOO3

(w/Production and

Reserves)

\section{Undiscovered Deposit Type}

1) 21b Porphyry Mo (low F)

\section{Number of Undiscovered Deposits} at Probability Percentile

$95 \quad 90 \quad 50 \quad 10 \quad 05$

$0 \quad 0 \quad 0 \quad 0.1$

Grade/Tonnage

Model?

Yes

Tract $\left(\mathrm{km}^{2}\right): \quad 62$ total: 42 in $\mathrm{BC}$ and 20 in $\mathrm{KC}$

Comments: Reconnaissance geologic mapping and geochemical sampling by USGS. Moderate amount of private prospecting and some exploration drilling. Close to tidewater, some parts steep and rugged; some thick brush and timber. Relatively small tract. $81 \%$ in USFS Misty Fiords Wilderness; $19 \%$ in USFS Tongass National Forest.

Discussion: Includes USGS OFR 91-010 tracts 02KC and 10BC. 


\section{Southeastern Alaska}

Iract Name: Chickamin Glacier

Iract No: $12 \mathrm{BC}$

Geology: Numerous sulfide deposits occur within $300 \mathrm{~m}$ above or below the contact of the Early Jurassic Texas Creek granodiorite and the metasedimentary and metavolcanic rocks of the Triassic age Hazelton Group.

Geochemistry: Stream-sediment anomalies: $\mathrm{Au}, \mathrm{Ag}, \mathrm{Cu}, \mathrm{Mo}, \mathrm{Pb}, \mathrm{Zn}$; bedrock anomalies: $\mathrm{Ag}, \mathrm{Cu}, \mathrm{Mo}$, $\mathrm{Pb}, \mathrm{Zn}$.

Geophysics:

Significant Deposits: BC 013-050; small production from BC026-047; these localities are included in an (w/Production and Reserves) aggregate inferred estimate of $690,000 \mathrm{mt}$ with $4.87 \mathrm{~g} / \mathrm{mt} \mathrm{Au}, 79.11 \mathrm{~g} / \mathrm{mt} \mathrm{Ag}, 0.13 \%$ $\mathrm{Cu}, 0.01 \% \mathrm{Mo}, 3.48 \% \mathrm{~Pb}, 0.03 \mathrm{~g} / \mathrm{mt} \mathrm{WO}_{3}, 0.30 \% \mathrm{Zn}$ for the whole Hyder subdistrict [Coldwell, 1990].

\section{Undiscovered Deposit Type}

1) $18 \mathrm{c} \mathrm{Zn}-\mathrm{Pb}$ skam

2) 22c Polymetallic vein

3) $18 b$ Cu skam

4) Metamorphosed sulfide

\section{Number of Undiscovered Deposits} at Probability Percentile

$\begin{array}{ccccc}95 & 90 & 50 & 10 & 05 \\ 0 & 1 & 2 & 3 & 5 \\ 0 & 0 & 0 & 1 & 2 \\ 0 & 0 & 0 & 0 & 1\end{array}$

Grade/Tonnage Model?

\begin{tabular}{|c|}
\hline Yes \\
\hline Yes \\
\hline Yes \\
\hline
\end{tabular}

Tract $\left(\mathrm{km}^{2}\right)$ : 199 total: 190 in $\mathrm{BC}$ and 9 in KC.

Comments: Reconnaissance and some detailed geologic mapping and geochemical sampling by USGS. Large amount of private prospecting and some exploration drilling. Remote, rugged; extensive glacier and permanent snow cover; old trails give acess from tidewater at Hyder. $62 \%$ in USFS Misty Fiords Wilderness; $38 \%$ in USFS Tongass National Forest.

Discussion: Includes USGS OFR 91-010 tracts $04 \mathrm{KC}$ and 12BC. 
Geology: Quartz and sulfide veins and shear zones cut Early Jurassic, Texas Creek granodiorite and Hazelton Group metavolcanic rocks.

Geochemistry: Bedrock anomalies: $\mathrm{Au}, \mathrm{Ag}, \mathrm{As}, \mathrm{Mo}, \mathrm{Sb}, \mathrm{Co}, \mathrm{Ni}$.

Geophysics:

Significant Depesits: $\mathrm{BC} 052-075$; $\mathrm{KC101-105}$; some production from $\mathrm{BC} 033,059,062$, and 075 ; these (w/Production and Reserves) localities are included in an aggregate inferred estimate of $690,000 \mathrm{mt}$ with 4.87 $\mathrm{g} / \mathrm{mt} \mathrm{Au}, 79.11 \mathrm{~g} / \mathrm{mt} \mathrm{Ag}, 0.13 \% \mathrm{Cu}, 0.01 \% \mathrm{Mo}, 3.48 \% \mathrm{~Pb}, 0.03 \mathrm{~g} / \mathrm{mt} \mathrm{WO}_{3}, 0.30 \%$ $\mathrm{Zn}$ for the whole Hyder subdistrict [Coldwell, 1990].

\section{Undiscovered Deposit Type}

1) $22 c$ Polymetallic vein

2) 21a Porphyry Cu-Mo

3) $28 \mathrm{a} .1$ Sierran kuroko massive sulfide

\section{Number of Undiscovered Deposits} at Probability Percentile

$\begin{array}{ccccc}95 & 90 & 50 & 10 & 05 \\ 0 & 0 & 0 & 1 & 2 \\ 0 & 0 & 0 & 0 & 1 \\ 0 & 0 & 0 & 0 & 1\end{array}$

\section{Grade/Tonnage} Model?

\begin{tabular}{|c|}
\hline Yes \\
\hline Yes \\
\hline Yes \\
\hline
\end{tabular}

Tract $\left(\mathrm{km}^{2}\right): \quad 68$ total: 44 in $\mathrm{BC}$ and 24 in $\mathrm{KC}$

Comments: Reconnaissance and some detailed geologic mapping and geochemical sampling by USGS. Large amount of private prospecting and some exploration drilling. Locally rugged; old trails and road give acess from tidewater at Hyder. $100 \%$ in USFS Tongass National Forest.

Discussion: Includes USGS OFR 91-010 tracts 05KC and 13BC. 
Geology: Paleozoic limestone/marble is intruded by Cretaceous felsic pluton; small high-grade $\mathrm{Pb}-\mathrm{Ag}$ replacement deposits occur locally.

Geochemistry: . Stream-sediment anomalies: Pb, Sb.

\section{Geophysics:}

Significant Deposits: CR001: Small high-grade $\mathrm{Pb}-\mathrm{Ag}$ replacement deposits in limestone/marble; more (w/Production and Reserves) than $100 \mathrm{~T}$ of Ag-bearing ore produced in early 1900 's.

\section{Undiscovered Deposit Type}

1) 19a Polymetallic replacement

\section{Number of Undiscovered Deposits} at Probability Percentile

95

90

50

0
0
10 05
Grade/Tonnage Model?

$\operatorname{Tract}\left(\mathrm{km}^{2}\right): \quad 73$

Comments: Reconnaissance geologic mapping and geachemical sampling by USGS. Moderate amount of private prospecting and some exploration drilling in 1970's. Locally rugged and steep; close to tidewater. $100 \%$ in USFS Coronation Island Wildemess.

Discussion: 
Geology: Descon Formation graywacke and minor carbonate rocks intruded by Cretaceous felsic pluton, are permissive for skam and porphyry deposits.

Geochemistry: Stream-sediment anomalies: $\mathrm{Pb}, \mathrm{Sb}$.

Geophysics:

\section{Significant Deposits:}

(w/Production and Reserves)

\section{Undiscovered Deposit Type}

1) $22 \mathrm{c}$ Polymetallic vein

2) $18 \mathrm{c} \mathrm{Zn-Pb} \mathrm{skam}$

3) 17.1 B.C.-Ak Porphyry Cu

4) 16 Climax Mo

\section{Number of Undiscovered Deposits} at Probability Percentile

$\begin{array}{lllll}95 & 90 & 50 & 10 & 05\end{array}$

- . - .

- $\quad$ - $\quad$ -

- $\quad-\quad-$

- $\quad-$
Grade/Tonnage Model?

\begin{tabular}{|c|}
\hline Yes \\
\hline Yes \\
\hline Yes \\
\hline Yes \\
\hline
\end{tabular}

$\operatorname{Tract}\left(\mathrm{km}^{2}\right): \quad 44$

Comments: Reconnaissance geologic mapping and geochemical sampling by USGS. Locally rugged and steep; local heavy timber; close to tidewater. $100 \%$ in USFS Coronation Island Wilderness.

Discussion: No estimates because of insufficient data. 
Geology: Silurian-age turbidites and minor volcanic rocks in a locally heterogeneous section that elsewhere contains some volcanogenic massive sulfide deposits.

Geochemistry: Stream-sediment anomalies: scattered low-level Zn.

\section{Geophysics:}

Signlficant Deposits: CROO3

(w/Production and

Reserves)

Undiscovered Deposit Type

1) $24 b$ Besshi massive sulfide

\section{Number of Undiscovered Deposits}

\section{at Probability Percentile}

$\begin{array}{lllll}95 & 90 & 50 & 10 & 05\end{array}$

$\begin{array}{lllll}0 & 0 & 0 & 0 & 1\end{array}$

\section{Grade/Tonnage}

Model?

Yes

Tract $\left(\mathrm{km}^{2}\right): \quad 649$ total: 523 in $\mathrm{CR}$ and 126 in PE

Comments: Reconnaissance geologic mapping and geochemical sampling by USGS. Small amount of prospecting. Moderately rugged and steep; local heavy timber and brush; some logging roads. Large tract. $100 \%$ in USFS Tongass National Forest.

Discussion: Includes USGS OFR 91-010 tracts O9PE and 03CR. 
Geology: Silurian-Ordovician-age andesitic breccia with minor limestone is intruded by large felsic Cretaceous-age pluton; environment permissive for U,Th, or REE..

Geochemistry: Stream-sediment anomalies: scattered Nb, La.

\section{Geophysics:}

\section{Significant Deposits:}

(w/Production and Reserves)

\section{Number of Undiscovered Deposits}

\section{Undiscovered Deposit Type}

1) Felsic plutonic $U$

2) 11d Th-RE veins

\begin{tabular}{ccccc}
\multicolumn{5}{c}{ at Probability Percentile } \\
95 & 90 & 50 & 10 & 05 \\
0 & 0 & 0 & 0 & 1 \\
0 & 0 & 0 & 0 & 1
\end{tabular}

Grade/Tonnage

Model?

No

Yes

\section{$\operatorname{Tract}\left(\mathrm{km}^{2}\right): 68$}

Comments: Reconnaissance geologic mapping and geochemical sampling by USGS. Small amount of prospecting. Moderately rugged and steep; local heavy timber and brush; some logging roads; close to tidewater. $100 \%$ in USFS Tongass National Forest.

Discussion: 
Geology: Alaskan-type ultramafic-mafic pluton of mid-Cretaceous age intrudes flysch and volcanic rocks of Gravina overlap assemblage.

Geochemistry: Stream-sediment anomalies: $\mathrm{Cr}$.

Geophysics: There must be a significant aeromagnetic anomaly associated with this pluton.

Significant Depesits: CR165: 1,000,000,000 T Ti-bearing, 18-20\% Fe ore; 907,1875,000 T w/ 19.0\% Fe (w/Production and [Coldwell, 1990].

Reserves)

\section{Undiscovered Deposit Type}

1) 9 Alaskan PGE

\section{Number of Undiscovered Deposits}

\begin{tabular}{lllll}
\multicolumn{2}{c}{ at Probability Percentile } & \\
25 & 90 & 50 & 10 & 05
\end{tabular}

0 0 00001

\section{Grade/Tonnage} Model?

No

Tract $\left(\mathrm{km}^{2}\right): 61$

Comments: Reconnaissance geologic mapping and geochemical sampling by USGS; some detailed mapping by USGS and others. Little prospecting for PGE-group metals. Moderately rugged and steep; local heavy timber and brush; close to tidewater. 100\% in USFS Tongass National Forest.

Discussion: 
Geology: Au-bearing quartz veins and parallel shear zones cross-cut low-grade metamorphic Mesozoic or Paleozoic pelitic and felsic(?) rocks.

Geochemistry: Stream-sediment anomalies: $\mathrm{Au}, \mathrm{Sb}, \mathrm{Pb}$.

Geophysics:

Significant Deposits: CR166-172; KC004-014. Production uncertain, but several mines in the CR

(w/Production and quadrangle produced hundeds to thousands of T Au ore from 1910-1930's; also a Reserves) few thousand oz Au produced from KCOO4 in 1898-1917. These localities included in aggregated inferred estimate of $282,000 \mathrm{mt} \mathrm{w} / 11.89 \mathrm{~g} / \mathrm{mt} \mathrm{Au}$ [Coldwell, 1990].

\section{Undiscovered Deposit Type}

1) 36a Low-sulfide Au-quartz vein

2) $22 \mathrm{c}$ Polymetallic vein

\section{Number of Undiscovered Deposits} at Probability Percentile

$\begin{array}{ccccc}95 & 90 & 50 & 10 & 05 \\ 0 & 1 & 3 & 7 & 12 \\ 0 & 0 & 0 & 0 & 1\end{array}$

\section{GraderTonnage}

Model?

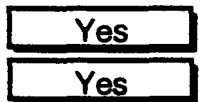

Tract $\left(\mathrm{km}^{2}\right): \quad 145$

Comments: Reconnaissance geologic mapping and geochemical sampling by USGS. Moderate to substantial amount of prospecting. Moderately rugged and steep; local heavy timber and brush; close to tidewater; limited outcrop. $100 \%$ in USFS Tongass National Forest.

Discussion: 
Geology: Large outcrop area of Ordovician, Devonian, and Mississippian sedimentary and volcanic rocks.

Geochemistry: Stream-sediment anomalies: $\mathrm{Cu}$, numerous; $\mathrm{Pb}, \mathrm{Zn}, \mathrm{Ba}, \mathrm{Ag}$ scattered.

\section{Geophysics:}

Significant Deposits: CR004-006, 012, 030, 031, 049, 063, 095-099, 127; DE008.

(w/Production and

Reserves)

\section{Undiscovered Deposit Type}

1) 28 a.1 Sierran kuroko massive sulfide

2) 22c Polymetallic vein

3) 17.1 BC-AK Porphyry $\mathrm{Cu}$

4) $18 b$ Cu skam
Number of Undiscovered Deposits at Probability Percentile

$95 \quad 90 \quad 50 \quad 10 \quad 05$

\begin{tabular}{ll|lll}
0 & 1 & 2 & 3 & 5
\end{tabular}

$\begin{array}{lllll}0 & 3 & 6 & 8 & 10\end{array}$

$\begin{array}{lllll}0 & 0 & 1 & 2 & 3\end{array}$

$\begin{array}{lllll}0 & 1 & 2 & 4 & 6\end{array}$
Grade/Tonnage

Model?

\begin{tabular}{|c|}
\hline Yes \\
\hline Yes \\
\hline Yes \\
\hline Yes \\
\hline
\end{tabular}

Tract $\left(\mathrm{km}^{2}\right): \quad 1,608$ total: 1,606 in $\mathrm{CR}, 1$ in DE, and 1 in $\mathrm{KC}$.

Comments: Reconnaissance geologic mapping and geochemical sampling by USGS. Moderate amount of prospecting. Moderately rugged and steep; local heavy timber and brush; close to tidewater; some logging roads. Very large tract. $2 \%$ in USFS Karta River Wilderness, $40 \%$ in Alaska Native lands, $58 \%$ in USFS Tongass National Forest.

Discussion: Includes USGS OFR 91-010 tracts 03DE, 10CR, and $11 \mathrm{KC}$. 


\section{Southeastern Alaska}

Tract Name: Kasaan Bay-Salt Chuck

Iract No: $11 \mathrm{CR}$

Geology: Multi-phase, Silurian Alaskan-type mafic-ultramafic pluton intrudes Paleozoic rocks; it contains a magmatic Cu-PGE-Au deposit.

Geochemistry: Stream-sediment anomalies: $\mathrm{Cu}$.

Geophysics: There must be an aeromagnetic anomaly associated with this pluton.

Significant Deposits: CR064, 066, 069-094; production from CR067 was about 300,000 T w/ $0.9 \% \mathrm{Cu}$, (w/Production and Reserves) $0.02 \mathrm{oz} / \mathrm{T} \mathrm{Au}, 0.1 \mathrm{oz} / \mathrm{T} \mathrm{Ag}, 0.05 \mathrm{oz} / \mathrm{T} \mathrm{Pd}$; $\mathrm{CR} 067$ estimated to contain $165,000 \mathrm{mt}$ inferred w/ $0.22 \mathrm{~g} / \mathrm{mt} \mathrm{Au}, 3.69 \mathrm{~g} / \mathrm{mt} \mathrm{Ag,} 0.02 \mathrm{~g} / \mathrm{mt} \mathrm{Pd,} 0.59 \% \mathrm{Cu}$ [Coldwell, 1990].

\section{Undiscovered Deposit Type}

1) 9 Alaskan PGE

\section{Number of Undiscovered Deposits at Probability Percentile}

$95 \quad 90 \quad 50 \quad 10 \quad 05$

\section{Grade/Tonnage}

Model?

No

Tract $\left(\mathrm{km}^{2}\right): \quad 16$

Comments: Detailed geologic mapping and reconnaissance geochemical sampling by USGS. Moderate amount of prospecting. Moderately rugged and steep; local heavy timber and brush; close to tidewater; logging and State roads; limited exposure. Very small tract. $100 \%$ in USFS Tongass National Forest.

Discussion: No estimate because of small size of tract. 
Geology: Paleozoic felsic to intermediate plutons intrude Lower Paleozoic calcareous metasedimentary rocks; Fe-Cu skarn deposits present.

Geochemistry: Strearn-sediment anomalies: $\mathrm{Cu}, \mathrm{Pb}, \mathrm{Zn}$; scattered.

Geophysics:

Significant Deposits: CR064, 066, 069-094. Production from several mines 1900- 1917 estimated to be (w/Production and Reserves) about 274,4000 T w/ 12.78 mlllion lbs Cu, 57,800 oz Ag. Resource estimates: 3.02 million T. Coldwell (1990) indicates an aggregate of $11,323,000 \mathrm{mt} w / 0.12 \mathrm{~g} / \mathrm{mt}$ $\mathrm{Au}, 0.26 \mathrm{~g} / \mathrm{mt} \mathrm{Ag}, 0.09 \% \mathrm{Cu}$, and $19.53 \% \mathrm{Cu}$ for all localities.

\section{Undiscovered Deposit Type}

1) $18 b$ Cu skarn

2) 18d Fe skarn

\section{Number of Undiscovered Deposits} at Probability Percentile

$\begin{array}{ccccc}95 & 90 & 50 & 10 & 05 \\ 0 & 1 & 2 & 3 & 5 \\ 0 & 1 & 2 & 3 & 5\end{array}$

Grade/Tonnage Model?

Yes

Tract $\left(\mathrm{km}^{2}\right): \quad 196$

Comments: Detailed geologic mapping and geochemical sampling by USGS. Moderate amount of prospecting. Locally rugged and steep; local heavy timber and brush; close to tidewater; logging roads in W part; limited exposure. $65 \%$ in Alaska Native lands, $35 \%$ in USFS Tongass National Forest.

\section{Discussion:}


Geology: Cretaceous pluton intrudes Silurian-Ordovician Descon Formation metasedimentary rocks; Mo-bearing porphyry system and vein deposits present.

Geochemistry: Stream-sediment anomalies: Mo, Ba, Cu, scattered. Au reported in old drill holes.

Geophysics:

Significant Deposits: CR009-011.

(w/Production and

Reserves)

\section{Undiscovered Deposit Type}

1) 21b Porphyry Mo (low F)

2) $22 c$ Polymetallic vein

\section{Number of Undiscovered Deposits}

at Probability Percentile

95

0 .

0

90

50

10

05

0

0

0

0

1

2
Grade/Tonnage

Model?

\begin{tabular}{c}
\hline Yes \\
\hline Yes \\
\hline
\end{tabular}

$\operatorname{Tract}\left(\mathrm{km}^{2}\right): \quad 85$

Comments: Reconnaissance geologic mapping and geochemical sampling by USGS. Some exploration drilling on CR010. Moderate amount of prospecting. Locally rugged and steep; local heavy timber and brush; close to tidewater; logging roads in West part; limited exposure. $100 \%$ in USFS Tongass National Forest.

\section{Discussion:}


Tract Name: San Juan Bautista Island

Geology: Small Cretaceous granitic pluton; some associated sulfide occurrences.

Geochemistry: Stream-sediment anomalies: $\mathrm{Pb}, \mathrm{Zn}, \mathrm{Cu}$, scattered.

Geophysics:

Significant Deposits: CR013, 014

(w/Production and

Reserves)

Undiscovered Deposit Type

1) 17.1 BC-AK Porphyry $\mathrm{Cu}$

2) 22c Polymetallic vein
Number of Undiscovered Deposits at Probability Percentile

$\begin{array}{lllll}95 & 90 & 50 & 10 & 05\end{array}$

$\begin{array}{lllll}0 & 0 & 0 & 0 & 1\end{array}$

0

0

0

1

\section{Grade/Tonnage}

Model?

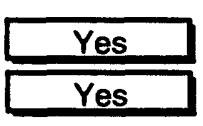

Tract $\left(\mathrm{km}^{2}\right): \quad 21$

Comments: Semi-detailed geologic mapping and geochemical sampling of shoreline by USGS; reconnaissance inland. Some exploration drilling on one prospect in 1970's. Moderate amount of prospecting. Locally rugged and steep; local heavy timber and brush; close to tidewater. Small tract. $100 \%$ in USFS Tongass National Forest.

Discussion: 
Geology: Syenitic and(or) granitic plutons intrude Lower and Middle Paleozoic volcanic and sedimentary rocks.

Geochemistry: Stream-sediment anomalies: Nb, Be, Y, La.

\section{Geophysics:}

Significant Deposits: CR015

(w/Production and

Reserves)

\section{Number of Undiscovered Deposits}

Undiscovered Deposit Type

1) Felsic plutonic $U$

2) 11d Th-RE veins

3) 21b Porphyry Mo (low F)

\section{at Probability Percentile}

$\begin{array}{lllll}95 & 90 & 50 & 10 & 05\end{array}$

$\begin{array}{lllll}0 & 0 & 0 & 0 & 1\end{array}$

$\begin{array}{lllll}0 & 0 & 0 & 0 & 1\end{array}$

$\begin{array}{lllll}0 & 0 & 0 & 0 & 1\end{array}$
Grade/Tonnage

Model?

\begin{tabular}{|c|}
\hline No \\
\hline Yes \\
\hline Yes \\
\hline
\end{tabular}

Tract $\left(\mathrm{km}^{2}\right): \quad 157$

Comments: Reconnaissance and some semi-detailed geologic mapping and geochemical sampling by USGS. Small amount of prospecting. Locally rugged and steep; local heavy timber and brush; close to tidewater; some logging roads. $100 \%$ in Alaska Native lands.

Discussion: 
Geology: Lower Paleozoic andesitic breccia and some Middle Paleozoic carbonate rocks are intruded by Cretaceous-age pluton; known Mo minerals.

Geochemistry: Stream-sediment anomalies: Mo.

\section{Geophysics:}

Significant Deposits; (a): CR018-020; (B): CR025 (w/Production and Reserves)

\section{Undiscovered Deposit Type}

1) 21b Porphyry Mo (low F)
Number of Undiscovered Deposits at Probability Percentile $\begin{array}{lllll}95 & 90 & 50 & 10 & 05\end{array}$

$0 \quad 0 \quad 0 \quad 0 \quad 1$

Grade/Tonnage Model?

$\operatorname{Tract}\left(\mathrm{km}^{2}\right): 233$

Comments: Reconnaissance and some semi-detailed geologic mapping and geochemical sampling by USGS. Moderate amount of prospecting, including drilling on one prospect in 1970's. Locally rugged and steep; local heavy timber and brush; close to tidewater; some State and logging roads. Moderate size tract. $50 \%$ in Alaska Native lands, $20 \%$ in USFS Karta River Wildemess, $30 \%$ in USFS Tongass National Forest.

Discussion: 


\section{Southeastern Alaska}

Tract Name: Pin Peak

Tract No: $\quad 17 C R$

Geology: Lower Paleozoic andesitic breccia and some Middle Paleozoic carbonate rocks are intruded by Cretaceous(?)- or Paleozoic(?) pluton; possible polymetallic veins.

Geochemıstry: Stream-sediment anomalies: Mo.

Geophysics:

Significant Deposits: CR016, 017, 021-024.

(w/Production and

Reserves)

\section{Undiscovered Deposit Type}

1) 21b Porphyry Mo (low F)

2) $22 c$ Polymetallic vein
Number of Undiscovered Deposits

\section{at Probability Percentlle}

$\begin{array}{lllll}95 & 90 & 50 & 10 & 05\end{array}$

$\begin{array}{lllll}0 & 0 & 0 & 0 & 1\end{array}$

$0 \quad 0 \quad 0 \quad 0 \quad 1$
Grade/Tonnage Model?

\begin{tabular}{|c|}
\hline Yes \\
\hline Yes \\
\hline
\end{tabular}

Tract $\left(\mathrm{km}^{2}\right): \quad 60$

Comments: Reconnaissance and some semi-detailed geologic mapping and geochemical sampling by USGS. Moderate amount of prospecting. Locally rugged and steep; local heavy timber and brush; close to tidewater; some State and logging roads. Small size tract. $25 \%$ in Alaska Native lands, $25 \%$ in USFS Karta River Wilderness, $50 \%$ in USFS Tongass National Forest.

Discussion: 
Tract Name: Maybeso Creek

Geology: Polymetallic veins in Ordovician shale and graywacke.

Geochemistry: Stream-sediment anomalies: $\mathrm{Zn}, \mathrm{Pb}, \mathrm{Mo}, \mathrm{Cu}$, widespread.

\section{Geophysics:}

Significant Deposits: CR026-029, 032-048. Production: several thousand oz Au in 1900-1940.

(w/Production and

Reserves)

\section{Undiscovered Deposit Type}

1) 21b Porphyry Mo (low F)

2) $22 c$ Polymetallic vein
Number of Undiscovered Deposits at Probability Percentile

$\begin{array}{lllll}95 & 90 & 50 & 10 & 05\end{array}$

$\begin{array}{lllll}0 & 0 & 0 & 0 & 1\end{array}$

$\begin{array}{lllll}0 & 1 & 3 & 6 & 8\end{array}$

GraderTonnage

Model?

Yes

Tract $\left(\mathrm{km}^{2}\right): 90$

Comments: Reconnaissance and some semi-detailed geologic mapping and geochemical sampling by USGS. Intermittent prospecting. Locally rugged and steep; local heavy timber and brush; close to tidewater; some State and logging roads. Small size tract. $15 \%$ in Alaska Native lands, $25 \%$ in USFS Karta River Wilderness, $60 \%$ in USFS Tongass National Forest.

Discussion: 
Geology: Mesozoic or Paleozoic pluton intrudes Silurian-Ordovician Descon Formation metasedimentary rocks; Mo-porphyry system and polymetallic vein environment..

Geochemistry: Stream-sediment anomalies: Nb, La.

\section{Geophysics:}

Significant Depositsi None

(w/Production and

Reserves)

\section{Undiscovered Deposit Type}

1) 21a Porphyry Cu-Mo

2) $22 c$ Polymetallic vein

3) Felsic plutonic $U$

4) 11d Th-RE veins
Number of Undiscovered Deposits at Probability Percentile

$95 \quad 90 \quad 50 \quad 10 \quad 05$

$0 \quad 0 \quad 0 \quad 0 \quad 1$

$0 \quad 0 \quad 0 \quad 001$

$0 \quad 0 \quad 0 \quad 001$

$0 \quad 0 \quad 0 \quad 0 \quad 1$
Grade/Tonnage Model?

\begin{tabular}{|c|}
\hline Yes \\
\hline Yes \\
\hline No \\
\hline Yes \\
\hline
\end{tabular}

$\operatorname{Tract}\left(\mathrm{km}^{2}\right): \quad 141$

Comments: Reconnaissance geologic mapping and geochemical sampling by USGS. Intermittent prospecting. Locally rugged and steep; local heavy timber and brush; close to tidewater. $100 \%$ in USFS Tongass National Forest.

Discussion: 
Tract Name: Trocadero Bay-Cholmondeley Sound

Geology: Low-grade Late Proterozoic-Early Paleozoic(?) Wales Group metamorphic rocks contain conformable sulfide lenses.

Geochemistry: Stream-sediment anomalies: $\mathrm{Ba}, \mathrm{Zn}, \mathrm{Pb}, \mathrm{Cu}$; scattered.

\section{Geophysics:}

Significant Deposits: CR050-058, 100, 102-105, 117, 122, 123, 125, 126, 128-131, 140, 143, 144.

(w/Production and Reserves)

Production: None. Reserves: For CR102: $76,000 \mathrm{mt}$ inferred w/ $2.06 \mathrm{~g} / \mathrm{mt} \mathrm{Au}, 10.28$ $\mathrm{g} / \mathrm{mt} \mathrm{Ag}, 1.71 \% \mathrm{Cu}, 0.93 \% \mathrm{Zn}$ [Coldwell, 1990]; for CR126: 49,000 mt inferred w/ $100 \%$ barite.

\section{Undiscovered Deposit Type}

1) 28 a Kuroko massive sulfide

\section{Number of Undiscovered Deposits at Probability Percentile}

$\begin{array}{lllll}95 & 90 & 50 & 10 & 05\end{array}$

\begin{tabular}{ll|lll}
0 & 1 & 2 & 3 & 5
\end{tabular}

\section{Grade/Tonnage} Model?

Yes

Tract $\left(\mathrm{km}^{2}\right): \quad 833$ total: 796 in CR and 37 in DE

Comments: Reconnaissance geologic mapping and geochemical sampling by USGS. Some prospecting. Low relief; local heavy timber and brush; close to tidewater; State and logging roads. Large tract. $38 \%$ in Alaska Native lands; $0.5 \%$ in South prince of Wales Wilderness; $61.5 \%$ in USFS Tongass National Forest.

Discussion: Includes USGS OFR 91-010 tracts 04DE and 20CR. 


\section{Southeastern Alaska}

Tract Name: Copper Mountain

Iract No: $21 \mathrm{CR}$

Geology: Paleozoic carbonate rocks are intruded by Cretaceous-age granodiorite plutons; numerous Fe and Cu skarn deposits.

Geochemistry: Stream-sediment anomalies: $\mathrm{Ba}, \mathrm{Zn}, \mathrm{Pb}, \mathrm{Cu}$.

Geophysics:

Significant Deposits; CR101, 106-121. Production: From 1902-1922, from several mines totaled 10 million (w/Production and Reserves) Ibs $\mathrm{Cu}, 280,000$ oz Ag, 7,000 oz Au. Resenves: For CR112: 65,000T w/ 45\% Fe, $0.75 \% \mathrm{Cu}$; also for CR112 [Coldwell, 1990]: $590,000 \mathrm{mt} \mathrm{w/} 0.34 \mathrm{~g} / \mathrm{mt} \mathrm{Au}, 2.70 \mathrm{~g} / \mathrm{mt}$ $\mathrm{Ag}, 0.75 \% \mathrm{Cu}, 45.2 \% \mathrm{Fe}$.

Undiscovered Deposit Type

1) $18 \mathrm{~b}$ Cu skarn

2) 18d Fe skam
Number of Undiscovered Deposits at Probability Percentile

$95 \quad 90 \quad 50 \quad 10 \quad 05$

$\begin{array}{lllll}0 & 0 & 0 & 1 & 2\end{array}$

$0 \quad 0 \quad 0 \quad 1 \quad 2$

Grade/Tonnage Model?

\begin{tabular}{|c|}
\hline Yes \\
\hline Yes \\
\hline
\end{tabular}

$\operatorname{Tract}\left(\mathrm{km}^{2}\right): \quad 116$

Comments: Reconnaissance and detailed geologic mapping and geochemical sampling by USGS.

Some prospecting. Locally steep; local heavy timber and brush; close to tidewater. $40 \%$ in Alaska Native lands; $60 \%$ in USFS Tongass National Forest.

Discussion: 
Geology: Jurassic-age syenite pluton intrudes Late Proterozoic and Early Paleozoic(?) Wales Group metasedimentary and metavolcanic rocks; REE deposit environment.

Geochemistry: Stream-sediment anomalies: REE.

Geophysics: Aeroradioactivity anomaly.

Significant Deposits: CR132.

(w/Production and

Reserves)

Number of Undiscovered Deposits

\section{Undiscovered Deposit Type}

1) Felsic plutonic $U$

2) 11d Th-RE veins

\section{at Probability Percentile}

$95 \quad 90 \quad 50 \quad 10 \quad \underline{90}$

$\begin{array}{llllll}0 & 0 & 0 & 1 & 2\end{array}$

\begin{tabular}{ll|lll}
0 & 0 & 0 & 1 & 2
\end{tabular}

\section{Grade/Tonnage}

Model?

\begin{tabular}{|c|}
\hline No \\
\hline Yes \\
\hline
\end{tabular}

$\operatorname{Tract}\left(\mathrm{km}^{2}\right): \quad 31$

Comments: Reconnaissance and detailed geologic mapping and geochemical sampling by USGS. Extensive prospecting. Locally very steep; local heavy timber and brush; close to tidewater. Small tract. $30 \%$ in Alaska Native lands; $70 \%$ in USFS Tongass National Forest.

Discussion: 
Geology: Late Proterozic and Early Cambrian(?) Wales Group marble contains veins.

Geochemistry: Stream-sediment anomalies: Mo, Zn, Cu.

Geophysics:

Significant Deposits: CR133-137, 139-142,153-163. Production: 1900-1932: 1,000 to 5,000 oz Au, mainly (w/Production and from CR153 (Valpariso)

Reserves)

\section{Undiscovered Deposit Type}

1) 22c Polymetallic vein

2) $36 a$ Low-sulfide Au-quartz vein
Number of Undiscovered Deposits at Probability Percentile

$\begin{array}{lllll}95 & 90 & 50 & 10 & 05\end{array}$

$\begin{array}{lllll}0 & 0 & 1 & 2 & 4\end{array}$

$\begin{array}{lllll}0 & 0 & 1 & 2 & 5\end{array}$

\section{Grade/Tonnage} Model?

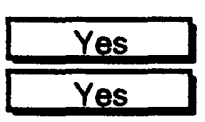

Tract $\left(\mathrm{km}^{2}\right): \quad 92$ total: 83 in CR and 9 in KC.

Comments: Reconnaissance and detailed geologic mapping and geochemical sampling by USGS.

Some prospecting. Low to moderate relief; local heavy timber and brush; close to tidewater; some logging roads. $27 \%$ in Alaska Native lands; $73 \%$ in USFS Tongass National Forest.

Discussion: Includes USGS OFR 91-010 tracts 12KC and 23CR. 


\section{Southeastern Alaska}

Tract Name: Northern Dall Island

Iract No: 24CR

Geology: Paleozoic sedimentary and volcanic rocks are intruded by Cretaceous-age granitic bodies.

Geochemistry: Stream-sediment anomalies: $\mathrm{Mo}, \mathrm{Zn}, \mathrm{Pb}, \mathrm{Sb}, \mathrm{Ba}$.

\section{Geophysics:}

Significant Deposits: CR059-062. Only production has been of marble. for cement.

(w/Production and

Reserves)

\section{Undiscovered Deposit Type}

1) 22c Polymetallic vein

2) $18 \mathrm{C} \mathrm{Zn-Pb} \mathrm{skarn}$

3) 21b Porphyry Mo (low F)
Number of Undiscovered Deposits

at Probability Percentile

$95 \quad 90 \quad 50 \quad 10 \quad 05$

$\begin{array}{lllll}0 & 0 & 0 & 0 & 1\end{array}$

$\begin{array}{lllll}0 & 0 & 0 & 0 & 1\end{array}$

$\begin{array}{lllll}0 & 0 & 0 & 0 & 1\end{array}$

\section{Grade/Tonnage}

Model?

\begin{tabular}{|c|}
\hline Yes \\
\hline Yes \\
\hline Yes \\
\hline
\end{tabular}

Iract $\left(\mathrm{km}^{2}\right): \quad 276$ total: 226 in CR and 50 in DE.

Comments: Reconnaissance geologic mapping and geochemical sampling by USGS. Some prospecting. Locally very steep; local heavy timber and brush; close to tidewater; some logging roads. $45 \%$ in Alaska Native lands; $55 \%$ in USFS Tongass National Forest.

Discussion: Includes USGS OFR 91-010 tracts O2DE and 24CR. 
Tract Name: Southeast Sukkwan tsland

Iract No: 25CR

Geology: Lower Paleozoic sedimentary rocks are intruded by Pennsylvanian-age syenite pluton.

Geochemistry: Stream-sediment anomalies: Nb, Be, Y.

Geophysics:

Significant Deposits: CR124

(w/Production and

Reserves)

\section{Undiscovered Deposit Type}

1) Felsic plutonic $U$

2) 11d Th-RE veins (
Number of Undiscovered Deposits at Probabillty Percentile

$\begin{array}{lllll}95 & 90 & 50 & 10 & 05\end{array}$

$\begin{array}{lllll}0 & 0 & 0 & 0 & 1\end{array}$

$\begin{array}{lllll}0 & 0 & 0 & 0 & 1\end{array}$

Grade/Tonnage

Model?

No

Yes

Tract $\left(\mathrm{km}^{2}\right): \quad 23$

Comments: Reconnaissance geologic mapping and geochemical sampling by USGS. Generally low relief, but locally very steep; local heavy timber and brush; close to tidewater, some State and logging roads. Small tract: $35 \%$ in Alaska Native lands; $65 \%$ in USFS Tongass National Forest.

Discussion: 
Geology: Cretaceous granitic plutons intrude Late Proterozoic and Early Paleozoic(?) Wales Group metasedimentary and metavolcanic rocks; porphyry and vein environment.

Geochemıstry: Stream-sediment anomalies: Mo.

\section{Geophysics:}

Significant Deposits: None

(w/Production and Reserves)

\section{Number of Undiscovered Deposits}

Undiscovered Deposit Type

1) 21b Porphyry Mo (low F)

2) $22 \mathrm{c}$ Polymetallic vein

3) $18 \mathrm{c} \mathrm{Zn-Pb} \mathrm{skam}$

$\begin{array}{ccccc}95 & 90 & 50 & 10 & 05 \\ 0 & 0 & 0 & 1 & 2 \\ 0 & 0 & 0 & 0 & 1 \\ 0 & 0 & 0 & 0 & 1\end{array}$

Grade/Tonnage

Model?

\begin{tabular}{|c|}
\hline Yes \\
\hline Yes \\
\hline Yes \\
\hline
\end{tabular}

Tract $\left(\mathrm{km}^{2}\right): 275$ total: 214 in CR, 52 in DE, 06 in PA, and 03 in KC.

Comments: Reconnaissance geologic mapping and geochemical sampling by USGS. Some industry prospecting and drilling. Locally very steep; local heavy timber and brush; close to tidewater. $100 \%$ in USFS Tongass National Forest.

Discussion: Includes USGS OFR 91-010 tracts 07DE, 01PA, 26CR, and 13KC. 
Geology: Felsic volcanic lenses in Late Proteozoic and Early Paleozoic(?) Wales Group contain massive sulfide deposits.

Geochemistry: Stream-sediment anomalies: $\mathrm{Ba}, \mathrm{Zn}, \mathrm{Pb}, \mathrm{Cu}$.

\section{Geophysics:}

Significant Deposits: CR145-152. Production from CR 149: from 1902-1909, at least 1.4 million lbs Cu, (w/Production and $15,000 \mathrm{oz} \mathrm{Ag}$.

Reserves)

\section{Number of Undiscovered Deposits}

Undiscovered Deposit Type

1) 28 a Kuroko massive sulfide

\section{at Probability Percentile}

$95 \quad 90 \quad 50 \quad 10 \quad 05$

$\begin{array}{lllll}0 & 0 & 1 & 2 & 3\end{array}$

Grade/Tonnage

Model?

Yes

Tract $\left(\mathrm{km}^{2}\right): 78$

Comments: Reconnaissance geologic mapping and geochemical sampling by USGS. Intense industry prospecting and drilling. Locally very steep; local heavy timber and brush; close to tidewater. Small tract. $100 \%$ in USFS Tongass National Forest.

Discussion: 
Geology: Lower and Middle Paleozoic volcanic and clastic sedimentary rocks and some marble are poorly exposed.

\section{Geochemistry:}

Geophysics: Prominent aeromagnetic high anomaly surrounded by several low anomalies suggest the existence of a concealed intrusion.

Significant Deposits: None

(w/Production and

Reserves)

\section{Undiscovered Deposit Type}

1) 21a Porphyry Cu-Mo

2) $18 \mathrm{~b}$ Cu skam

3) $22 \mathrm{c}$ Polymetallic vein

\section{Number of Undiscovered Deposits} at Probability Percentile

$95 \quad 90 \quad 50 \quad 10 \quad 05$

- $\quad-\quad-\quad-10$

\section{Grade/Tonnage \\ Model?}

\begin{tabular}{|c|}
\hline Yes \\
\hline Yes \\
\hline Yes \\
\hline
\end{tabular}

$\operatorname{Tract}\left(\mathrm{km}^{2}\right): \quad 114$

Comments: Reconnaissance geologic mapping and geochemical sampling by USGS. Minor amount of prospecting. Locally very steep; local heavy timber and brush; close to tidewater. $100 \%$ in USFS Tongass National Forest.

Discussion: No estimate because of inadequate data. 
Tract Name: Forrester Island

Iract No: 01DE

Geology: Granodiorite of probable Cretaceous age contains porphyry Mo deposit.

Geochemistry:

Geophysics:

Significant Deposits: DE001, 002.

(w/Production and

Reserves)

Undiscovered Deposit Type

1) 21b Porphyry Mo (low F)
Number of Undiscovered Deposits

at Probability Percentile

$95 \quad 90 \quad \underline{50} \quad 10 \quad 05$

$\begin{array}{lllll}0 & 0 & 0 & 0 & 1\end{array}$

\section{Grade/Tonnage}

Model?

Yes

Tract $\left(\mathrm{km}^{2}\right): \quad 11$

Comments: Reconnaissance geologic mapping and geochemical sampling by USGS. Low amount of prospecting. Locally very steep; local heavy timber and brush; close to open-ocean tidewater. Small tract. $100 \%$ in USFWS Forrester Island Wildlife Refuge.

Discussion: 
Tract Name: Kassa Inlet

Iract No: 05DE

Geology: Small Paleozoic plutons in Late Proterozoic and Cambrian(?) Wales Group rocks may be permissive for U-Th deposits.

Geochemistry: Stream-sediment anomalies: Nb, Y.

\section{Geophysics:}

Signlficant Deposits: None

(w/Production and

Reserves)

\section{Number of Undiscovered Deposits}

\section{Undiscovered Deposit Type}

1) Felsic plutonic $U$

2) 11d Th-RE veins at Probability Percentile

$\begin{array}{lllll}95 & 90 & 50 & 10 & 05\end{array}$

\begin{tabular}{ll|lll}
0 & 0 & 0 & 0 & 1
\end{tabular}

\begin{tabular}{ll|lll}
0 & 0 & 0 & 0 & 1
\end{tabular}

\section{Grade/Tonnage}

Model?

\begin{tabular}{|c|}
\hline No \\
\hline Yes \\
\hline
\end{tabular}

$\operatorname{Tract}\left(\mathrm{km}^{2}\right): \quad 26$

Comments: Reconnaissance and some detailed geologic mapping and geochemical sampling by USGS and by ADGGS. Moderate amount of prospecting. Mostly close to tidewater; moderate relief. Small tract. 50\% in USFS South Prince of Wales Wilderness, $50 \%$ in USFS Tongass National Forest.

Discussion: 
Geology: Middle Jurassic peralkaline granite has associated veins, dikes, and pegmatites that contain $U, T h$, and REE minerals.

\section{Geochemistry:}

Geophysics:

Significant Deposits: DE028, 030-040. Production: DE039: about $110,000 \mathrm{~T}$ w/ about $1.3 \%$ U3O8 from (w/Production and 1955 to 1975. Reserves: All of these localities are included in an inferred estimate of

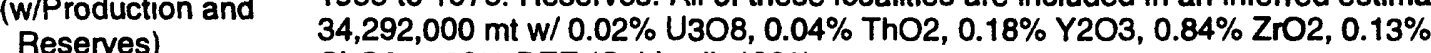
Reserves) CbO2, 0.32\% REE (Coldwell, 1990).

\section{Undiscovered Deposit Type}
1) Felsic plutonic $U$
2) 11d Th-RE veins

\section{Number of Undiscovered Deposits} at Probability Percentile

$\begin{array}{ccccc}95 & 90 & 50 & 10 & 05 \\ 0 & 0 & 0 & 0 & 1 \\ 0 & 0 & 1 & 2 & 5\end{array}$

GraderTonnage Model?

No

$\operatorname{Tract}\left(\mathrm{km}^{2}\right): \quad 33$

Comments: Detailed geologic mapping and some geochemical sampling by USGS; also some samping by USBM. Extensive prospecting. Locally very steep; local heavy timber and brush; road to tidewater from tract. Small tract. $100 \%$ in USFS Tongass National Forest.

\section{Discussion:}


Geology: Minor Cretaceous granitic intrusions cut Late Proterozoic and Early Paleozoic Wales Group rocks; polymetallic veins in the wallrocks.

\section{Geochemistry:}

\section{Geophysics:}

Significant Deposits: DE003-007.

(w/Production and

Reserves)

\section{Undiscovered Deposit Type}

1) 28 a Kuroko massive sulfide

2) 22c Polymetallic vein

\section{Number of Undiscovered Deposits} at Probability Percentile

$\begin{array}{lllll}95 & 90 & 50 & 10 & 05\end{array}$

\begin{tabular}{ll|lll}
0 & 0 & 0 & 0 & 1
\end{tabular}

$\begin{array}{llllll}0 & 1 & 2 & 4 & 8\end{array}$

Grade/Tonnage Model?

\begin{tabular}{|c|}
\hline Yes \\
\hline Yes \\
\hline
\end{tabular}

Tract $\left(\mathrm{km}^{2}\right): \quad 436$

Comments: Reconnaissance and some detailed geologic mapping and geochemical sampling by USGS. Some prospecting. Locally very steep; local heavy timber and brush; close to tidewater. Large tract. $40 \%$ in Alaska Native lands, $60 \%$ in USFS Tongass National Forest.

\section{Discussion:}


Geology: Lower Paleozoic, Descon Formation rocks contain scattered massive sulfide occurrences.

\section{Geochemistry:}

Geophysics:

Significant Deposits: DE009-015, 017.

(w/Production and

Reserves)

Undiscovered Deposit Type

1) 28a Kuroko massive sulfide

Number of Undiscovered Deposits at Probability Percentile

$95 \quad 90 \quad 50 \quad 10 \quad \underline{05}$

$\begin{array}{lllll}0 & 0 & 0 & 1 & 2\end{array}$

Grade/Tonnage

Model?

Yes

Tract $\left(\mathrm{km}^{2}\right): \quad 117$

Comments: Reconnaissance and some detailed geologic mapping and geochemical sampling by USGS. Some recent prospecting. Generally low relief; local heavy timber and brush; close to tidewater. Moderate-size tract. $90 \%$ in USFS Southern Prince of Wales Island Wilderness, $10 \%$ in USFS Tongass National Forest.

Discussion: 
Tract Name: Southeasternmost Prince of Wales Island

Geology: Complex geology with diverse Lower Paleozoic metasedimentary and metavolcanic rocks cut by granitic bodies of different ages.

\section{Geochemistry:}

\section{Geophysics:}

Significant Deposits: DE018-031; PR001, 002; DE023-025 area contains 40 million tonnes hypothetical (w/Production and resources (Nokleberg and others, 1995) Reserves)

\section{Undiscovered Deposit Type}

1) $22 \mathrm{c}$ Polymetallic vein

2) 21a Porphyry Cu-Mo

3) Felsic plutonic $U$

4) 11d Th-RE veins

5) 10 Carbonatite

\section{Number of Undiscovered Deposits} at Probability Percentile

$\begin{array}{lllll}95 & 90 & 50 & 10 & 05\end{array}$

$\begin{array}{lllll}0 & 0 & 0 & 0 & 1\end{array}$

$\begin{array}{lllll}0 & 0 & 0 & 0 & 1\end{array}$

$\begin{array}{lllll}0 & 0 & 0 & 1 & 2\end{array}$

$\begin{array}{lllll}0 & 0 & 0 & 1 & 2\end{array}$

$\begin{array}{lllll}0 & 0 & 0 & 0 & 1\end{array}$

GraderTonnage Model?

\begin{tabular}{|c|}
\hline Yes \\
\hline Yes \\
\hline No \\
\hline Yes \\
\hline Yes \\
\hline
\end{tabular}

Tract $\left(\mathrm{km}^{2}\right): \quad 16$ total: 131 in DE and 15 in PR.

Comments: Reconnaissance and some detailed geologic mapping and geochemical sampling by USGS. Some recent prospecting. Generally steep; local heavy timber and brush; close to tidewater. Moderate-size tract. $100 \%$ in USFS Tongass National Forest.

Discussion: Includes USGS OFR 91-010 tracts 02PR and 10DE. 
Geology: Complex clastic, carbonate, and volcanic rock section of Permian and Late Triassic(?) age is intruded and metamorphosed by Tertiary and Cretaceous granitic plutons; stratiform massive sulfide deposits present.

Geochemistry; Stream-sediment anomalies: $\mathrm{Cu}, \mathrm{Mo}, \mathrm{Pb}, \mathrm{Co}, \mathrm{Cr}, \mathrm{Ni}, \mathrm{Hg}$.

\section{Geophysics:}

Significant Deposits: JU012, 013.

(w/Production and

Reserves)

\section{Undiscovered Deposit Type}

1) $28 a .1$ Sierran kuroko massive sulfide
Number of Undiscovered Deposits at Probability Percentile

$\begin{array}{lllll}95 & 90 & 50 & 10 & 05\end{array}$

$\begin{array}{lllll}0 & 0 & 0 & 1 & 2\end{array}$

Grade/Tonnage Model?

Tract $\left(\mathrm{km}^{2}\right): 63$

Comments: Reconnaissance geologic mapping and geochemical sampling by USGS. Some recent prospecting by USBM. Generally steep and rugged; some glacier cover; local heavy timber and brush; close to tidewater. Relatively small and well-known tract. $100 \%$ in USNPS Glacier Bay National Park.

Discussion: Recent investigations (D.A. Brew and others, 1991) indicate that the carbonate and other sedimentary rocks are Permian, and that the amygdaloidal volcanic rocks are probably Late Triassic. 
Geology: Paleozoic clastic and carbonate rocks are intruded by Cretaceous and Tertiary granitic dikes; fracturing and alteration are quite local; Mo-Cu porphyry stockwork and disseminated mineralization in Westpart of tract.

Geochemistry: Stream sediment anomalies: Mo.

\section{Geophysics:}

Significant Deposits: JU001-003.

(w/Production and

Reserves)

\section{Undiscovered Deposit Type}

1) 21b Porphyry Mo (low F)

2) Porphyry $\mathrm{Cu}$ (skarn-related)

3) 22c Polymetallic vein

\section{Number of Undiscovered Deposits} at Probability Percentile

$\begin{array}{lllll}95 & 90 & 50 & 10 & 05\end{array}$

$\begin{array}{lllll}0 & 0 & 0 & 0 & 1\end{array}$

$\begin{array}{llllll}0 & 0 & 0 & 0 & 1\end{array}$

0

0

0

05
1
1
1

\section{Grade/Tonnage} Model?

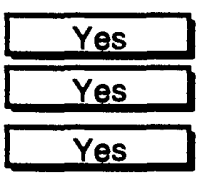

Tract $\left(\mathrm{km}^{2}\right): 576$ total: 330 in JU, 104 in MF, and 142 in SK.

Comments: Reconnaissance geologic mapping and geochemical sampling by USGS. Some recent prospecting by USBM. Extensive private prospecting of areas deglaciated in 1960's and 1970 's. Locally steep and rugged, but generally moderate relief; some glacier cover; local heavy timber and brush; in part close to tidewater. $89 \%$ in USNPS Glacier Bay National Park; $11 \%$ in USFS Tongass National Forest.

Discussion: Includes USGS OFR 91-010 tracts 02JU, 08MF, and 06 SK. 
Geology: Slightly metamorphosed Paleozoic volcanic and carbonate rocks; minor Cu mineralization in volcanics.

Geochemistry:

Geophysics:

Significant Deposits: JU004, 005, 009, 010.

(w/Production and

Reserves)

Undiscovered Deposit Type

1) 28 a.1 Sierran kuroko massive sulfide

\section{Number of Undiscovered Deposits} at Probability Percentile

$\begin{array}{lllll}95 & 90 & 50 & 10 & 05\end{array}$

$0 \quad 0 \quad 00100$

Grade/Tonnage Model?

Yes

$\operatorname{Iract}\left(\mathrm{km}^{2}\right): \quad 132$

Comments: Reconnaissance geologic mapping and geochemical sampling by USGS. Probably essentially unprospected. Remote, steep and rugged; some glacier cover; local heavy timber and brush; in part close to tidewater. $85 \%$ in USNPS Glacier Bay National Park; $5 \%$ in USFS Tongass National Forest.

\section{Discussion:}


Geology: Paleozoic metamorphosed clastic, carbonate, and volcanic rocks of west-dipping structure contain stratiform skarn mineralization.

Geochemistry: Bedrock anomalies: $\mathrm{As}, \mathrm{Ni}, \mathrm{Ag}, \mathrm{Cu}, \mathrm{Zn}$.

\section{Geophysics:}

Significant Deposits: JU006-008; SK016a,b.

(w/Production and

Reserves)

\section{Undiscovered Deposit Type}

1) $18 b$ Cu skarn

2) $18 \mathrm{c} \mathrm{Zn-Pb} \mathrm{skarn}$

\section{Number of Undiscovered Deposits} at Probability Percentile

\begin{tabular}{cc|ccc}
95 & 90 & 50 & 10 & 05 \\
0 & 0 & 0 & 0 & 1 \\
0 & 0 & 1 & 1 & 2
\end{tabular}

Grade/Tonnage

Model?

\begin{tabular}{|c|}
\hline Yes \\
\hline Yes \\
\hline
\end{tabular}

Tract $\left(\mathrm{km}^{2}\right): \quad 335$ total: 246 in JU and 89 in SK.

Comments: Reconnaissance geologic mapping and geochemical sampling by USGS and by USBM. Moderate to high level of recent prospecting, including some exploration drilling. Locally steep and rugged; local heavy timber and brush; in part close to tidewater. $11 \%$ in USFS Endicott River Wildemess; $13 \%$ in Alaska State lands; $76 \%$ in USFS Tongass National Forest.

Discussion: Includes USGS OFR 91-010 tracts 08SK and 04JU. 
Geology: Silurian graywacke and argillite with some carbonate layers are intruded by scattered granodioritic plugs and stocks with possibly associated veins.

Geochemistry: Stream-sediment anomalies: $\mathrm{Ag}, \mathrm{Be}, \mathrm{Co}, \mathrm{Hg}, \mathrm{Ni}, \mathrm{Pb}, \mathrm{Zn}, \mathrm{Au}$.

\section{Geophysics:}

Significant Deposits: JU018, 022-024.

(w/Production and

Reserves)

\section{Undiscovered Deposit Type}

1) 22c Polymetallic vein
Number of Undiscovered Deposits at Probability Percentile

$\begin{array}{lllll}95 & 90 & 50 & 10 & 05\end{array}$

$\begin{array}{lllll}0 & 0 & 0 & 1 & 2\end{array}$

Grade/Tonnage

Model?

Yes

Tract $\left(\mathrm{km}^{2}\right): \quad 524$

Comments: Reconnaissance geologic mapping and geochemical sampling by USGS and some by USBM and private industry. In part remote, locally very steep and rugged; some glacier cover; local heavy timber and brush. $70 \%$ in USNPS Glacier Bay National Park; $5 \%$ in Alaska Native lands; $25 \%$ in USFS Tongass National Forest.

\section{Discussion:}


Geology: Silurian graywacke and argillite with some carbonate beds are intruded by a large granodiorite pluton: permissive skam environment.

Geochemistry:

Geophysics: Aeromagnetic anomaly associated with pluton.

Significant Deposits: None

(w/Production and Reserves)

Undiscovered Deposit Type

1) $18 b$ Cu skam

2) $18 \mathrm{c} \mathrm{Zn}-\mathrm{Pb}$ skam

\section{Number of Undiscovered Deposits} at Probability Percentile

$\begin{array}{lllll}95 & 90 & 50 & 10 & 05\end{array}$

$\begin{array}{lllll}0 & 0 & 0 & 0 & 1\end{array}$

0

0

0
1

\section{Grade/Tonnage} Model?

\begin{tabular}{|c|}
\hline Yes \\
\hline Yes \\
\hline
\end{tabular}

$\operatorname{Tract}\left(\mathrm{km}^{2}\right): 246$

Comments: Reconnaissance geologic mapping and geochemical sampling by USGS and some by USBM and private industry. West part locally very steep and rugged; some glacier cover; local heavy timber and brush; in part close to tidewater. $10 \%$ in USNPS Glacier Bay National Park; $90 \%$ in USFS Tongass National Forest.

Discussion: No known deposits. 
Geology: Paleozoic carbonate and clastic rocks are intruded and hornfelsed by Cretaceous granodioirite plutons: permissive skarn environment.

\section{Geochemistry:}

Geophysics:

Significant Deposits: None

(w/Production and

Reserves)

\section{Undiscovered Deposit Type}

1) $18 b$ Cu skarn

2) $18 \mathrm{c} \mathrm{Zn}-\mathrm{Pb}$ skarn
Number of Undiscovered Deposits at Probability Percentile

$\begin{array}{ccccc}95 & 90 & 50 & 10 & 05 \\ 0 & 0 & 0 & 0 & 1 \\ 0 & 0 & 0 & 0 & 1\end{array}$

Grade/Tonnage

Model?

\begin{tabular}{|c|}
\hline Yes \\
\hline Yes \\
\hline
\end{tabular}

Tract $\left(\mathrm{km}^{2}\right): \quad 50$ total: 48 in JU, 02 in SI.

Comments: Reconnaissance geologic mapping and geochemical sampling by USGS. Locally steep and rugged; local heavy timber and brush; in part close to tidewater. $100 \%$ in USFS Tongass National Forest.

Discussion: No known deposits. Includes USGS OFR 91-010 tracts 09SI and 07JU. 
Geology: Highly deformed and locally metamorphosed Late Triassic mafic and intermediate volcanic rocks, fine-grained clastic rocks, and ultramafic masses host isignificant massive sulfide, $\mathrm{Ni}-\mathrm{Cu}$ magmatic segregation, and polymetallic vein deposits. SIO90 (Pyrola) is best known deposit in SI quad part.

Geochemistry: Stream-sediment anomalies: very abundant and varied. Bedrock anomalies: very abundant and varied.

\section{Geophysics:}

Significant Deposits: (w/Production and Reserves)

JU028-045; S1088-091. Production:.JU032, 034, and 037: significant from vein deposits; JU033: significant from Ni-Cu deposit; JU044 (Greens Creek): greater than 6 million oz Ag and 24,000 oz Au. Reserves: JU033 (Mertie): 560,000 T w/ $0.35 \% \mathrm{Cu}, 0.34 \% \mathrm{Ni}, 0.15 \% \mathrm{Co}$; USBM inferred estimate is $508,000 \mathrm{mt} w / 0.35 \% \mathrm{Cu}$, $0.34 \% \mathrm{Ni}, 0.15 \%$ Co (Coldwell, 1990); JU044 (Greens Creek): As of 1990: 3,500,000 $\mathrm{T} w / 3.9 \% \mathrm{~Pb}, 9.7 \% \mathrm{Zn}, 23.8 \mathrm{oz} / \mathrm{T} \mathrm{Ag}, 0.18$ oz/T Au. As of March 1996: 80 million $\mathrm{T}$ w/ $21 \mathrm{oz} / \mathrm{mt} \mathrm{Ag}$ and $0.011 \mathrm{ox} \mathrm{mt} \mathrm{Ag}$ (Thompson, 1996).

\section{Undiscovered Deposit Type}

1) $28 \mathrm{a} .1$ Sierran kuroko massive sulfide

2) $7 \mathrm{a}$ Synorogenic-synvolcanic Ni-Cu

3) 22c Polymetallic vein

\section{Number of Undiscovered Deposits} at Probability Percentile

\begin{tabular}{cc|ccc}
95 & 90 & 50 & 10 & 05 \\
0 & 0 & 0 & 1 & 3 \\
0 & 0 & 0 & 0 & 1 \\
0 & 0 & 0 & 0 & 1
\end{tabular}

Grade/Tonnage Model?

\begin{tabular}{|c|}
\hline Yes \\
\hline Yes \\
\hline Yes \\
\hline
\end{tabular}

Tract $\left(\mathrm{km}^{2}\right): \quad 628$ total: 409 in JU and 219 in SI.

Comments: Reconnaissance and some semi-detailed geologic and geochemical mapping by USGS; intense private prospecting in past; close to tidewater in part; parts low relief, others steep and rugged; some heavy brush and timber; relatively large tract. About $49 \%$ in USFS Admiralty Island Monument and most of that in Kootznoowoo Wilderness, but some is Alaska Native lands; $3 \%$ in USFS Young Lake Wildemess; about $48 \%$ in USFS Tongass National Forest (but some small part of this is privately owned).

Discussion: Includes USGS OFR 91-010 tract 09JU and part of 13SI. Tract 13SI is geologic continuation to south. 
Tract Name: (A) Kensington-Jualin , and (B) Eagle River-Juneau

Iract No: $11 \mathrm{JU}$

Geology: (A): Au-bearing quartz veins in shear zones in mid-Cretaceous quartz monzonite pluton and in adjacent hornfelsed Late Triassic(?) basalt. (B): Au-bearing quartz veins in phyllite, slate, greenstone, greenschist, and metagabbro of Permian(?) through middle Cretaceous age that are variously deformed and metamorphosed, also in highly altered quartz monzonite sills of mid- or Late Cretaceous age.

\section{Geochemistry:}

Geophysics:

Significant Deposits; A): JU047-055,057-059; at (B): JU060-134, TR006-008. Production: (A): Significant (w/Production and production from deposits JU047,049-051, 055, 058; Kensington (JU051) now close Reserves) to renewed production; Jualin (JU058) now being explored and reopened. (B): very large production in past from deposits JU072 (Eagle River), JU101 (Alaska-Juneau), JU125 (Treadwell Group); Alaska-Juneau (JU101) now being evaluated for renewed production; also $100 \mathrm{oz}$ Au produced from TR008 (Enterprise). Reserves: (A): JU051 has inferred 20,000,000 T w/ 0.14 oz/T Au; JU058 reported to have 1,190,000 T w/ 0.236 oz/T Au; (B): JU101 has 100,000,000 T w/ 0.047 oz/T Au; TR008 has USBM estimate of $47,600 \mathrm{mt} \mathrm{w} / 6.86 \mathrm{~g} / \mathrm{mt} \mathrm{Au}$ (Coldwell, 1990).

\section{Undiscovered Deposit Type}

1) 36 a Low-sulfide Au-quartz vein

2) 28a.1 Sierran kuroko massive sulfide

\section{Number of Undiscovered Deposits}

$$
\text { at Probability Percentile }
$$

$\begin{array}{ccccc}95 & 90 & 50 & 10 & 05 \\ 0 & 1 & 2 & 3 & 5 \\ 0 & 0 & 1 & 2 & 3\end{array}$

Grade/Tonnage Model?

\begin{tabular}{|c|}
\hline Yes \\
\hline Yes \\
\hline
\end{tabular}

Tract $\left(\mathrm{km}^{2}\right): \quad 897$ total: 801 in JU and 96 in TR; (A) and (B) not recorded separately.

Comments: Reconnaissance and detailed geologic mapping and geochemical sampling by USGS,, USBM, and private industry. Locally steep and rugged; local heavy timber and brush; close to tidewater; large tract. About 33\% in USFS Tongass National Forest; $67 \%$ in Alaska State lands, Alaska Native lands, Alaska State park, City and Borough of Juneau lands, and private lands.

Discussion: Includes USGS OFR 91-010 tracts 01TR and 11JU. 


\section{Southeastern Alaska}

Tract Name: Juneau Icefield

Iract No: 13JU

Geology: Late Proterozoic(?), Paleozoic, and younger(?) metamorphosed clastic, carbonate, and volcanic rocks are intruded and homfelsed by Tertiary plutons; permissive skarn environment.

\section{Geochemistry:}

Geophysics:

Significant Deposits: None

(w/Production and Reserves)

\section{Number of Undiscovered Deposits}

Undiscovered Deposit Type
1) $18 b$ Cu skam
2) $18 \mathrm{c} \mathrm{Zn-Pb} \mathrm{skam}$ at Probability Percentile

95

\begin{tabular}{cc|ccc}
95 & 90 & 50 & 10 & 05 \\
0 & 0 & 0 & 1 & 2 \\
0 & 0 & 0 & 1 & 2
\end{tabular}

\section{Grade/Tonnage} Model?
Yes
Yes

Tract $\left(\mathrm{km}^{2}\right): \quad 506$

Comments: Reconnaissance and detailed geologic mapping and geochemical sampling by USGS. Essentially unprospected. Remote; exensive glacier cover; steep and rugged; large tract. $100 \%$ in USFS Tongass National Forest.

Discussion: 
Geology: Schist, gneiss, and minor marble of onginal Late Proterozoic(?), Paleozoic, and (or) Mesozoic age.

\section{Geochemistry:}

\section{Geophysics:}

Significant Deposits: KC084-087.

(w/Production and Reserves)

\section{Undiscovered Deposit Type}

1) $18 \mathrm{c} \mathrm{Zn-Pb} \mathrm{skarn}$

2) 22c Polymetallic vein

3) 18b Cu skarn

4) Metamorphosed sulfide

\section{Number of Undiscovered Deposits} at Probability Percentile

$\begin{array}{ccccc}95 & \mathbf{9 0} & \mathbf{5 0} & \mathbf{1 0} & \mathbf{0 5} \\ 0 & 0 & 0 & 0 & 1 \\ 0 & 0 & 0 & 1 & 2 \\ 0 & 0 & 0 & 0 & 1 \\ 0 & 0 & 0 & 0 & 1\end{array}$

Grade/Tonnage Model?

\begin{tabular}{|c|}
\hline Yes \\
\hline Yes \\
\hline Yes \\
\hline No \\
\hline
\end{tabular}

Tract $\left(\mathrm{km}^{2}\right)$ : 18 total: 130 in $\mathrm{KC}$ and 18 in $\mathrm{BC}$.

Comments: Reconnaissance geologic mapping and geochemical sampling by USGS. Essentially unprospected. Remote; some glacier cover; steep and rugged; local heavy timber and brush; large valleys. $1.00 \%$ in USFS Misty Fiords Wilderness.

Discussion: Includes USGS OFR 91-010 tracts 11BC and 03KC. 
Tract Name: Chickamin-Rudyerd

Iract No: 07KC

Geology: Schist, gneiss, and minor marble of Late Proterozoic(?), Paleozoic, and (or) Mesozoic age.

\section{Geochemistry:}

\section{Geophysics:}

Significant Deposits: KC088, 090-093.

(w/Production and

Reserves)

\section{Undiscovered Deposit Type}

Number of Undiscovered Deposits at Probability Percentile

1) 22c Polymetallic vein

2) $18 \mathrm{~b}$ Cu skam

$\begin{array}{lllll}95 & 90 & 50 & 10 & 05\end{array}$

$0 \quad 0 \quad 0 \quad 001$

3) Metamorphosed sulfide

$\begin{array}{lllll}0 & 0 & 0 & 1 & 2\end{array}$

Grade/Tonnage

Model?

\begin{tabular}{|c|}
\hline Yes \\
\hline Yes \\
\hline No \\
\hline
\end{tabular}

Tract $\left(\mathrm{km}^{2}\right): \quad 794$

Comments: Reconnaissance geologic mapping and geochemical sampling by USGS. Not heavily prospected. Mostly remote; some glacier cover; steep and rugged; in part close to tidewater; local heavy timber and brush; large tract. 100\% in USFS Misty Fiords Wilderness.

Discussion: 
Geology: Argillite, phyllite, greenschist, muscovite schist, and marble are intruded by metamorphosed aplite and granodiorite and by unmetamorphosed granodiorite and gabbro.

Geochemistry: Stream-sediment anomalies: Zn.

Geophysics:

Significant Deposits: KC015-017, 022-037, 096, 097. Production: 400 to $500 \mathrm{~T} \mathrm{Pb-Zn-Ag} \mathrm{ore} \mathrm{produced}$ (w/Production and Reserves) from KC022 in 1947. Reserves: at KC022: about 2,500 T w/ 6 to $7 \% \mathrm{~Pb}, 28 \% \mathrm{Zn}$; at KC025: $100,000 \mathrm{~T}$ w/ $7.5 \% \mathrm{Zn}, 1.0 \% \mathrm{Cu}$, and $100,000 \mathrm{~T}$ at lower grade. Both of these localities are included in an aggregated estimate of $93,000 \mathrm{mt} w / 0.05 \mathrm{~g} / \mathrm{mt}$ $\mathrm{Au}, 1.01 \% \mathrm{Cu}, 0.15 \% \mathrm{~Pb}, 7.96 \% \mathrm{Zn}$ (Coldwell, 1990).

\section{Undiscovered Deposit Type}

1) $36 a$ Low-sulfide Au-quartz vein

2) $22 c$ Polymetallic vein

3) $28 a .1$ Sierran kuroko massive sulfide

4) Metamorphosed sulfide
Number of Undiscovered Deposits at Probability Percentile

$\begin{array}{lllll}95 & 90 & 50 & 10 & 05\end{array}$

$\begin{array}{lllll}0 & 0 & 0 & 0 & 1\end{array}$

$\begin{array}{lllll}0 & 0 & 0 & 1 & 2\end{array}$

$\begin{array}{llllll}0 & 0 & 0 & 0 & 1\end{array}$

Grade/Tonnage Model?

\begin{tabular}{|c|}
\hline Yes \\
\hline Yes \\
\hline Yes \\
\hline No \\
\hline
\end{tabular}

Tract $\left(\mathrm{km}^{2}\right): \quad 1,709$

Comments: Reconnaissance geologic mapping and geochemical sampling by USGS. Some recent prospecting. Part of area is remote; locally steep and rugged; in part close to tidewater; some logging roads; local heavy timber and brush; very large tract. $30 \%$ in USFS Misty Fiords Wilderness; $20 \%$ in Alaska Native lands; $50 \%$ in USFS Tongass National Forest.

Discussion: 
Geology: Ti-bearing magnetite occurs in Cretaceous Alaskan-type ultramafic body that intrudes Paleozoic country rocks.

\section{Geochemistry:}

Geophysics: Aeromagnetic anomaly associated with ultramafic body.

Significant Deposits; KC038.

. (w/Production and

Reserves)

\section{Number of Undiscovered Deposits}

\section{Undiscovered Deposit Type} at Probability Percentile

1) 9 Alaskan PGE

$\begin{array}{lllll}95 & 90 & 50 & 10 & 05\end{array}$

\section{Grade/Tonnage \\ Model?}

Tract $\left(\mathrm{km}^{2}\right): \quad 11$

Comments: Reconnaissance geologic mapping and geochemical sampling by USGS. Some recent prospectirig. In part close to tidewater; local heavy timber and brush; very small tract. $100 \%$ in USFS Misty Fiords Wilderness.

Discussion: 
Geology: Gneiss, schist, and minor marble of Late Proterozoic(?), Paleozoic, and (or) Mesozoic age and pegmatite and gneissic quartz diorite are intruded by altered epizonal Miocene granite plutons and quartz porphyry dikes that contain disseminated $\mathrm{MoS}_{2}$.

Geochemistry: Stream-sediment anomalies: $\mathrm{Be}, \mathrm{Mo}, \mathrm{Nb}$.

\section{Geophysics:}

Significant Deposits: KC095. Reserves: at KC095: 1,500,000,000 T w/ 0.136\% Mo; also an inferred (w/Production and estimate of 1,360,778,000 mt w/ 0.14\% MoS2 (Coldwell, 1990). Reserves)

\section{Undiscovered Deposit Type}

1) 21b Porphyry Mo (low F)

2) 22c Polymetallic vein

3) $18 \mathrm{~b}$ Cu skam

\section{Number of Undiscovered Deposits} at Probability Percentile

$\begin{array}{ccccc}95 & 90 & 50 & 10 & 05 \\ 0 & 0 & 0 & 1 & 3 \\ 0 & 0 & 0 & 1 & 2 \\ 0 & 0 & 0 & 1 & 2\end{array}$

Grade/Tonnage Model?

\begin{tabular}{|c|}
\hline Yes \\
\hline Yes \\
\hline Yes \\
\hline
\end{tabular}

Tract $\left(\mathrm{km}^{2}\right): \quad 627$

Comments: Reconnaissance and some detailed geologic mapping and geochemical sampling by USGS; intense local prospecting. In part close to tidewater, generally rugged; large tract; local heavy timber and brush. $40 \%$ in USFS Misty Fiords Wilderness; $60 \%$ in non-Wilderness part of USFS Misty Fiords Wilderness.

\section{Discussion:}


Tract Name: (A) Southwestern Gravina Island, and (B) Eastern Annette Island

Iract No: $14 \mathrm{KC}$

Geology: Upper Triassic Puppets Formation metarhyolite and metadacite flows and tuff host massive, vein, and disseminated sulfide deposits.

\section{Geochemistry:}

\section{Geophysics:}

Significant Deposits: At (A): KC042, 043, 047-062, 064, 067-070; at (B) KC076-081, 083. Production: At (w/Production and KC056: small test shipment in early 1900's.

Reserves)

\section{Undiscovered Deposit Type}

1) $28 a .1$ Sierran kuroko massive sulfide

2) 22c Polymetallic vein
Number of Undiscovered Deposits at Probability Percentile

$\begin{array}{ccccc}95 & 90 & \mathbf{5 0} & \mathbf{1 0} & 05 \\ 0 & 0 & 1 & 2 & 4 \\ 0 & 0 & 0 & 1 & 2\end{array}$

Grade/Tonnage Model?

\begin{tabular}{c}
\hline Yes \\
\hline Yes \\
\hline
\end{tabular}

Tract $\left(\mathrm{km}^{2}\right): \quad 183$ total: 130 in (A) and 53 in (B).

Comments: Reconnaissance and locally detailed geologic and geophysical mapping and geochemical sampling by USGS; some local prospecting. In part close to tidewater; locally steep; local heavy timber and brush. $43 \%$ in Metlakatla Indian Reservation; $57 \%$ in USFS Tongass National Forest.

Discussion: 
Geology: Ti-bearing magnetite occurs in Cretaceous Alaskan-type ultramafic body that intrudes Paleozoic country rocks.

Geochemistry:

Geophysics: Aeromagnetic anomaly associated with ultramafic body.

Significant Deposits; KC074, 075.

(w/Production and Reserves)

Undiscovered Deposit Type

1) 9 Alaskan PGE

\section{Number of Undiscovered Deposits} at Probabillity Percentile

$95 \quad 90 \quad 50 \quad 10 \quad 05$

\section{Grade/Tonnage \\ Model?}

No

\section{Tract $\left(\mathrm{km}^{2}\right): \quad 21$}

Comments: Reconnaissance and some detailed geologic and geophysical mapping and geochemical sampling by USGS; some past and recent prospecting. Close to tidewater; low relief; poor outcrops; not much timber and brush; very small tract. $100 \%$ in Metlakatla Indian Reservation.

Discussion: 
Geology: Phyllite, schist, and greenschist intruded by metamorphosed diorite and by Tertiary leucogabbro host Au-quartz veins in vicinity of Tongass Narrows fault.

\section{Geochemistry:}

\section{Geophysics:}

Significant Deposits: KC18-021, 039-041, 044-046, 063, 066, 071, 072. Production: several thousand oz (w/Production and Reserves) Au produced before 1917 from all of tract. Reserves: these localities are included in an aggregated inferred estimate of $562,000 \mathrm{mt} \mathrm{w} / 10.47 \mathrm{~g} / \mathrm{mt} \mathrm{Au}$.

\section{Undiscovered Deposit Type}

1) 36a Low-sulfide Au-quartz vein

2) 22c Polymetallic vein

\section{Number of Undiscovered Deposits} at Probability Percentile

$\begin{array}{ccccc}95 & 90 & 50 & 10 & 05 \\ 0 & 0 & 1 & 2 & 4 \\ 0 & 0 & 0 & 0 & 1\end{array}$

Grade/Tonnage Model?

Yes

Tract $\left(\mathrm{km}^{2}\right): 224$

Comments: Reconnaissance and some detailed geologic and geophysical mapping and geochemical sampling by USGS; much old and some minor recent prospecting. Close to tidewater and, in part, to roads; locally steep; local thick timber and brush. $10 \%$ in Metlakatla Indian Reservation; $40 \%$ in Ketchikan City and private lands; $30 \%$ in Alaska Native lands, $20 \%$ in USFS Tongass National Forest.

Discussion: 
Tract Name: Boca de Quadra-Sitlan Island

Iract No: $18 \mathrm{KC}$

Geology: Paleozoic and Mesozoic metasedimentary and some metavolcanic rocks are intruded by Cretaceous granodiorite plutons and Cretaceous or Tertiary trondjhemite sills.

\section{Geochemistry:}

\section{Geophysics:}

Significant Deposits: None

(w/Production and

Reserves)

\section{Undiscovered Deposit Type}

1) 28a.1 Sierran kuroko massive sulfide

2) $22 c$ Polymetallic vein

\section{Number of Undiscovered Deposits} at Probability Percentile

$95 \quad 90 \quad 50 \quad 10 \quad 05$

$\begin{array}{lllll}0 & 0 & 0 & 0 & 1\end{array}$

$0 \quad 0 \quad 0 \quad 0 \quad 1$

\section{Grade/Tonnage}

Model?

\begin{tabular}{c}
\hline Yes \\
\hline Yes \\
\hline
\end{tabular}

Tract $\left(\mathrm{km}^{2}\right): \quad 292$ total: 172 in $\mathrm{KC}$ and 120 in PR.

Comments: Reconnaissance geologic mapping and geochemical sampling by USGS; little past or recent prospecting. Close to tidewater in part; moderately rugged; local thick timber and brush. $100 \%$ in USFS Misty Fiords Wilderness.

Discussion: An extension of tract $08 \mathrm{KC}$, which is to northwest, but without all of the deposit types expected in that tract. Includes USGS OFR 91-010 tracts 04PR and 18KC. 
Geology: Gneiss, schist, and minor marble intruded by pegmatite, gneissic quartz diorite, and granodiorite: schist derived from volcanic rocks is permissive massive sulfide environment.

Geochemistry:

Geophysics:

Significant Deposits: KC098-100.

(w/Production and

Reserves)

Undiscovered Deposit Type

Number of Undiscovered Deposits

at Probability Percentlle

$95 \quad 90 \quad 50 \quad 10 \quad 05$

Grade/Tonnage

Model?

1) Metamorphosed sulfide

Tract $\left(\mathrm{km}^{2}\right): \quad 56$

Comments: Reconnaissance geologic mapping and geochemical sampling by USGS; little past or recent prospecting. Close to tidewater in part; moderately rugged; local thick timber and brush. $100 \%$ in USFS Misty Fiords Wilderness.

Discussion: 
Geology: Layered cumulus-type gabbro body with some mineralization (known from float) that is similar to that in tract 09MF; intrudes metamorphosed Mesozoic flyschoid and volcanic rocks; many Cu-stained zones.

Geochemistry: Bedrock anomalies not far to West: $\mathrm{Co}, \mathrm{Cr}, \mathrm{Cu}, \mathrm{Ni}$.

Geophysics: Large aeromagnetic anomaly associated with pluton.

Significant Deposits; None.

(w/Production and

Reserves)

\section{Undiscovered Deposit Type}

1) 7 a Synorogenic-synvolcanic $\mathrm{Ni}-\mathrm{Cu}$
Number of Undiscovered Deposits

at Probability Percentile

$95 \quad 90 \quad 50 \quad 10 \quad 05$

$\begin{array}{lllll}0 & 0 & 1 & 2 & 3\end{array}$

Grade/Tonnage

Model?

$\operatorname{Tract}\left(\mathrm{km}^{2}\right): \quad 81$

Comments: Reconnaissance geologic mapping and geochemical sampling by USGS; essentially unprospected. Extremely remote and rugged; extensive glacier cover; no timber and brush. $100 \%$ in USNPS Glacier Bay National Park.

Discussion: 
Geology: Complex clastic, volcanic, and carbonate section of Permian(?) and (or) Triassic(?) age is intruded and metamorphosed by Tertiary and Cretaceous plutons and dikes in the vicinity of the Tarr Inlet suture zone; porphyry and massive sulfide deposit environment.

\section{Geochemistry:}

\section{Geophysics:}

Significant Deposits: MF023-025, 027, 029-031; SK001-003. Peserves: At MF027: USGS/USBM indicated (w/Production and Reserves) estimate of $270,000 \mathrm{~T} \mathrm{w} / 2.7 \% \mathrm{Cu}, 5.2 \% \mathrm{Zn}, 0.03 \mathrm{oz} / \mathrm{T} \mathrm{Au}, 1.0 \mathrm{oz} / \mathrm{T} \mathrm{Ag}$; and 530,000 $\mathrm{T} \mathrm{w} / 0.4 \% \mathrm{Cu}, 0.3 \% \mathrm{Zn}, 0.006 \mathrm{oz} / \mathrm{T} \mathrm{Au}, 0.35 \mathrm{oz} / \mathrm{T} \mathrm{Ag}$.

\section{Undiscovered Deposit Type}

1) 17.1 B.C.-Ak Porphyry $\mathrm{Cu}$

2) 28a.1 Sierran Kuroko massive sulfide

Number of Undiscovered Deposits at Probabillity Percentile

$\begin{array}{ccccc}95 & 90 & 50 & 10 & 05 \\ 0 & 0 & 1 & 2 & 2 \\ 0 & 0 & 1 & 2 & 3\end{array}$

GraderTonnage Model?

\begin{tabular}{|c|}
\hline Yes \\
\hline Yes \\
\hline
\end{tabular}

Iract $\left(\mathrm{km}^{2}\right): \quad 309$ total: 90 in SKand 219 in MF.

Comments: Reconnaissance geologic mapping and geochemical sampling by USGS and USBM; extensive prospecting by USBM. Close to tidewater; steep and rugged; extensive glacier cover; little timber and brush. $100 \%$ in NPS Glacier Bay National Park.

Discussion: Includes USGS OFR 91-010 tracts 05MF and 03SK. 
Geology: Narrow, discontinuous sulfide-bearing quartz veins in altered Cretaceous-age granitic rocks and hornfelsed Paleozoic clastic rocks in vicinity of Tarr Inlet suture zone.

Geochemistry: Stream-sediment anomalies: $\mathrm{Au}, \mathrm{Cu}$.

Geophysics:

Significant Deposits: MF032-042, 067. Production: About 7,150 oz Au produced during 1940's, mostly (w/Production and Reserves)

\section{Undiscovered Deposit Type}

1) 36a Low-sulfide Au-quartz vein

\section{Number of Undiscovered Deposits} at Probability Percentile

$95 \quad 90 \quad 50 \quad 10 \quad 05$

$\begin{array}{lllll}0 & 1 & 2 & 3 & 4\end{array}$

Grade/Tonnage Model?

Yes

$\operatorname{Tract}\left(\mathrm{km}^{2}\right): 74$

Comments: Reconnaissance geologic mapping and geochemical sampling by USGS and USBM; much earlier prospecting. Close to tidewater; locally steep and rugged; extensive glacier cover; little timber and brush. $100 \%$ in NPS Glacier Bay National Park.

Discussion: 


\section{Southeastern Alaska}

Tract Name: Muir Inlet

Tract No: 07MF

Geology: Paleozoic clastic and carbonate rocks are intruded by Cretaceous and Tertiary granitic dikes; fracturing and alteration are widespresd; Mo-Cu porphyry stockwork and disseminated deposits are known.

\section{Geochemistry:}

Geophysics:

Significant Deposits: MF074, 076-080; SK 006, 012. Reserves: At MF079: USGS/USBM indicated (w/Production and Reserves) estimated of $8,200,000 \mathrm{~T} w / 0.06 \% \mathrm{Mo}, 0.02 \% \mathrm{Cu}$; and $137,000,000 \mathrm{~T} \mathrm{w} / 0.04 \% \mathrm{Mo}$, $0.02 \% \mathrm{Cu}$; also inferred estimate of $9,100,000 \mathrm{~T} \mathrm{w} / 0.06 \% \mathrm{Mo}, 0.02 \% \mathrm{Cu}$.

\section{Undiscovered Deposit Type}

1) $21 \mathrm{a}$ Porphyry Cu-Mo

2) 18a Porphyry Cu (skarn-related)

3) $22 \mathrm{c}$ Polymetallic vein

4) 19a Polymetallic replacement

\section{Number of Undiscovered Deposits} at Probability Percentile

$\begin{array}{lllll}95 & 90 & 50 & 10 & 05\end{array}$

$\begin{array}{lllll}0 & 0 & 1 & 2 & 2\end{array}$

$\begin{array}{lllll}0 & 0 & 0 & 1 & 2\end{array}$

$\begin{array}{llllll}0 & 0 & 0 & 0 & 1\end{array}$

$\begin{array}{llllll}0 & 0 & 0 & 0 & 1\end{array}$

\section{Grade/tonnage} Model?

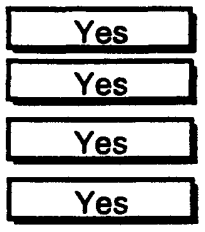

Tract $\left(\mathrm{km}^{2}\right): \quad 368$ total: 243 in MFand 25 in SK.

Comments: Reconnaissance geologic mapping and geochemical sampling by USGS and USBM; some prospecting by USBM; much earlier prospecting of areas deglaciated in 1960's and 1970 's. In part close to tidewater; locally steep and rugged, but generally moderate relief; some glacier cover. $100 \%$ in USNPS Glacier Bay National Park.

Discussion: Includes USGS OFR 91-010 tracts 05SK and 07MF. 


\section{Southeastern Alaska}

Tract Name: Crillon-La Perouse

Tract No: $09 \mathrm{MF}$

Geology: Layered cumulus-type Tertiary gabbro body intrudes metamorphosed Mesozoic flyschoid and volcanic rocks; known magmatic segregation $\mathrm{Ni}$-Cu deposit in peridotite at base of gabbro.

Geochemistry:

Geophysics:

Significant Deposits: MF005-007, 012-015. Reserves: At MF079: USGS/USBM indicated estimate of (w/Production and Reserves) $90,000,000 \mathrm{~T} w / 0.93 \% \mathrm{Ni}, 0.33 \% \mathrm{Cu}$, unspecified amount of PGE; also inferred estimate of $90,000,000 \mathrm{~T} w / 0.93 \% \mathrm{Ni}, 0.33 \% \mathrm{Cu}$, unspecified amount of PGE.

\section{Undiscovered Deposit Type}

1) 11d Th-RE veins (AP/THRE)
Number of Undiscovered Deposits at Probability Percentile

$\begin{array}{lllll}95 & 90 & 50 & 10 & 05\end{array}$

$\begin{array}{lllll}0 & 0 & 1 & 2 & 3\end{array}$

Grade/Tonnage Model?

Yes

Tract $\left(\mathrm{km}^{2}\right): 290$

Comments: Reconnaissance geologic mapping and some geochemical sampling by USGS; minor prospecting of areas deglaciated in 1950's. Very remote, very steep and rugged; very extensive glacier cover. $100 \%$ in USNPS Glacier Bay National Park, but patented claims cover part of deposit.

Discussion: 
Geology: Biotite schist and gneiss derived from Mesozoic flyschoid and volcanic rocks are intruded by Tertiary and Tertiary and (or) Cretaceous granitic and layered gabbroic stocks. The gabbro is mineralized.

Geochemistry: Stream-sediment anomalies: $\mathrm{Ba}, \mathrm{Cr}, \mathrm{Co}, \mathrm{Cu}, \mathrm{Ni}, \mathrm{Zn}, \mathrm{Hg}, \mathrm{Pb}$.

\section{Geophysics:}

Significant Deposits: MF018, 020.

(w/Production and

Reserves)

\section{Undiscovered Deposit Type}

1) 22c Polymetallic vein

2) 7 a Synorogenic-synvolcanic $\mathrm{Ni}-\mathrm{Cu}$
Number of Undiscovered Deposits at Probability Percentile

$\begin{array}{llllll}95 & 90 & 50 & 10 & 05\end{array}$

$\begin{array}{lllll}0 & 0 & 1 & 2 & 3\end{array}$

$0 \quad 0 \quad 0 \quad 0 \quad 1$

Grade/Tonnage Model?

Yes

Tract $\left(\mathrm{km}^{2}\right): \quad 437$

Comments: Reconnaissance geologic mapping and some geochemical sampling by USGS; minor prospecting. In part close to tidewater, somewhat remote, somewhat rugged; steep brushand timber-covered slopes. $100 \%$ in NPS Glacier Bay National Park.

\section{Discussion:}


Geology: Paleozoic carbonate, clastic, and minor volcanic rocks are intruded by voluminous Tertiary and Cretaceous granitic rocks; some skarn deposits near intrusions.

Geochemistry: Stream-sediment anomalies: $\mathrm{Cu}, \mathrm{Zn}, \mathrm{Pb}, \mathrm{Sn}, \mathrm{W}$.

\section{Geophysics:}

Significant Deposits: MF050b, 052-055, 057-061. Reserves: At MF054: USGS/USBM indicated estimate of (w/Production and $27,000 \mathrm{~T} w / 1.0 \% \mathrm{Cu}, 0.1 \mathrm{oz} / \mathrm{T} \mathrm{Au}, 2.0 \mathrm{oz} / \mathrm{T} \mathrm{Ag}$.

Reserves)

\section{Undiscovered Deposit Type}

1) $18 b$ Cu skarn

2) 22c Polymetallic vein

3) 21a Porphyry Cu-Mo

\section{Number of Undiscovered Deposits} at Probability Percentile

$\begin{array}{ccccc}95 & 90 & 50 & 10 & 05 \\ 0 & 0 & 1 & 2 & 3 \\ 0 & 0 & 1 & 2 & 3 \\ 0 & 0 & 0 & 0 & 1\end{array}$

\begin{tabular}{c} 
Grade/Tennage \\
Model? \\
\hline Yes \\
\hline Yes \\
\hline Yes \\
\hline
\end{tabular}

Tract $\left(\mathrm{km}^{2}\right): \quad 413$

Comments: Reconnaissance geologic mapping and some geochemical sampling by USGS; minor recent prospecting. In part close to tidewater; somewhat remote, somewhat rugged; steep brush- and timber-covered slopes. $85 \%$ in NPS Glacier Bay National Park; $15 \%$ in USFS Pleasant-Lemesurier Islands Wilderness.

Discussion: 
Geology: A): elongate lenses of serpentinite occur as tectonic slivers in phyllite and slate and contain minor chromite pods and disseminations. (B): magmatic segregations of chromite in serpentinized dunite of uncertain origin.

\section{Geochemistry:}

\section{Geophysics:}

Significant Deposits: (A) PA006-013; (B) PA016. Reserves: at PA016: 8 separate deposits contain a (w/Production and total of $30,000 \mathrm{~T}$ with 18 to $40 \% \mathrm{Cr} 2 \mathrm{O}$.

Reserves)

\section{Undiscovered Deposit Type}

1) 8a Podiform chromite (minor)

\section{Number of Undiscovered Deposits} at Probabillity Percentile

$\begin{array}{lllll}95 & 90 & 50 & 10 & 05\end{array}$

$\begin{array}{lllll}0 & 0 & 1 & 2 & 3\end{array}$

\section{Grade/Tonnage} Model?

Yes

Tract $\left(\mathrm{km}^{2}\right): \quad 32$ total: 28 in (A) and 4 in (B)

Comments: Known Deposits; permissive geology; both (A) and (B) are small tracts. Reconnaissance and some sem-detailed geologic and geochemical mapping by USGS; at (B) detailed geologic mapping and sampling by USGS. (A) is rugged and remote; (B) is close to tidewater with brush cover. $56 \%$ in USFS South Baranof Wildemess; $44 \%$ in USFS Tongass National Forest.

\section{Discussion:}


Geology: Dominantly middle Paleozoic graywacke, conglomerate, and some carbonate rocks; significant faults.

Geochemistry: Stream-sediment anomalies: Mo, Ba. Bedrock anomalies: $\mathrm{Pb}, \mathrm{Zn}, \mathrm{Mo}, \mathrm{Cr}, \mathrm{Ni}$, and $\mathrm{Co}$

Geophysics: Centered on aeromagnetic low between two highs.

Significant Deposits: None

(w/Production and

Reserves)

Undiscovered Deposit Type

1) $22 c$ Polymetallic vein(?)
Number of Undiscovered Deposits at Probability Percentile

$\begin{array}{lllll}95 & 90 & 50 & 10 & 05\end{array}$

$\begin{array}{lllll}0 & 0 & 0 & 1 & 2\end{array}$

Grade/Tonnage

Model?

Yes

Tract $\left(\mathrm{km}^{2}\right): \quad 239$

Comments: Reconnaissance and some semi-detailed geologic and geochemical mapping by USGS; minor exploration. Timber- and brush-covered; some logging roads. $100 \%$ in USFS Tongass National Forest. Permissive geology; prominent faults.

Discussion: 
Geology: Dominantly middle Paleozoic graywacke, conglomerate, and some carbonate rocks in SW one-third of tract; mixed volcanic, carbonate, and clastic Mesozoic rocks to northeast; barite masses and veins in north part of tract.

Geochemistry: Stream-sediment anomalies: $\mathrm{Pb}, \mathrm{Ba}, \mathrm{Zn}, \mathrm{Nb}, \mathrm{Cu}, \mathrm{Zn}, \mathrm{Pb}, \mathrm{La}, \mathrm{Nb}$. Bedrock anomalies: $\mathrm{Mo}, \mathrm{Cr}$, and $\mathrm{Ni}$.

Geophysics: Large aeromagnetic high with a deep source.

Significant Deposits: None

(w/Production and

Reserves)

\section{Undiscovered Deposit Type}

1) $28 a .1$ Sierran kuroko massive sulfide

2) 32a SE Missouri Pb-Zn (?)

\section{Number of Undiscovered Deposits} at Probability Percentile

\begin{tabular}{cc|ccc}
95 & 90 & 50 & 10 & 05 \\
0 & 0 & 0 & 0 & 1 \\
0 & 0 & 0 & 0 & 1
\end{tabular}

\section{GraderTonnage}

Model?

\begin{tabular}{|c|}
\hline Yes \\
\hline Yes \\
\hline
\end{tabular}

Tract $\left(\mathrm{km}^{2}\right): \quad 153$ total: 145 in PA and 8 in PE

Comments: Reconnaissance and some semi-detailed geologic and geochemical mapping by USGS; local intense exploration. Timber- and brush-covered; some logging roads. $84 \%$ in USFS Tongass National Forest; about $15 \%$ in Alaska Native lands; $1 \%$ in proposed USFS Rocky Pass Wilderness. Permissive geology, prominent faults.

Discussion: 
Geology: Cretaceous granodiorite plutons and dikes intrude middle Paleozoic graywacke, carbonate, and minor conglomerate; some large altered zones.

Geochemistry: Stream sediment anomalies: Mo, $\mathrm{W}$, and $\mathrm{Zn}$ in north part of tract; $\mathrm{Y}$ and $\mathrm{Nb}$ anomalies to south. Bedrock anomalies: weak $\mathrm{Mo}, \mathrm{Zn}, \mathrm{Cu}$, and $\mathrm{Co}$ in north part of tract; $\mathrm{As}, \mathrm{Ag}$, $\mathrm{Au}, \mathrm{Pb}, \mathrm{Cu}$ in southwest part; $\mathrm{Ag}, \mathrm{As}, \mathrm{Sb}, \mathrm{Zn}, \mathrm{Cu}, \mathrm{Pb}$ in southeast part.

\section{Geophysics:}

Significant Deposits: None (w/Production and Reserves)

\section{Undiscovered Deposit Type}

1) 21a Porphyry Cu-Mo

2) $22 c$ Polymetallic vein

\section{Number of Undiscovered Deposits} at Probability Percentile

$95 \quad 90 \quad 50 \quad 10 \quad 05$

$0 \quad 0 \quad 0 \quad 0 \quad 1$

$0 \quad 0 \quad 0 \quad 1 \quad 2$

\section{Grade/Tonnage}

Model?

Yes

Tract $\left(\mathrm{km}^{2}\right): \quad 277$ total: 263 in PA and 14 in PE

Comments: Permissive geology. Timber- and brush-covered; locally steep; in part close to tidewater. $100 \%$ in proposed USFS South Kuiu Wilderness. Includes tracts O4PE and 08PA.

\section{Discussion:}


Geology: Foliated, lineated, and hornfelsed Jurassic--Cretaceous graywacke and argillite are exposed over bujried Tertiary pluton.

Geochemistry: NURE Stream-sediment anomalies: $\mathrm{Cu}, \mathrm{As}, \mathrm{Au}, \mathrm{Pb}, \mathrm{Zn}$.

\section{Geophysics:}

Significant Deposits: PA017.

(w/Production and

Reserves)

\section{Number of Undiscovered Deposits}

\section{Undiscovered Deposit Type}

1) $22 c$ Polymetallic vein at Probability Percentile

95

$\begin{array}{llll}90 & 50 & 10 & 05\end{array}$

Grade/Tonnage Model?

$\begin{array}{lllll}0 & 0 & 0 & 1 & 2\end{array}$

Yes

Tract $\left(\mathrm{km}^{2}\right): \quad 594$

Comments: Reconnaissance and some detailed geologic mapping by USGS. NURE geochemical sampling. Very little prospecting. Mostly close to tidewater, most is steep and rugged. Large tract. About $40 \%$ in USFS South Baranof Wilderness, $60 \%$ in USFS Tongass National Forest.

Discussion: 


\section{Southesstern Alaska}

Tract Name: (A) Falls Lake, and (B) Lords Pocket

Iract No: 10PA

Geology: Mesozoic greenstone and metabasalt are variably metamorphosed; amphibolite facies in (A), lower greenschist facies in (B).

Geochemistry: Stream-sediment anomalies: $\mathrm{Cu}, \mathrm{Zn}, \mathrm{As}, \mathrm{Pb}$.

\section{Geophysics:}

Significant Deposits: None.

(w/Production and

Reserves)

\section{Undiscovered Deposit Type}

1) $28 a .1$ Sierran kuroko massive sulfide

$\begin{array}{ccccr}\text { Number of Undiscovered Deposits } \\ \text { at Probability Percentile } \\ 95 & 90 & 50 & 10 & 05 \\ 0 & 0 & 0 & 0 & 1\end{array}$

Grade/Tonnage

Model?

Yes

Tract $\left(\mathrm{km}^{2}\right): \quad 80$ total: 48 in (A) and 32 in (B).

Comments: Reconnaissance geologic mapping by USGS. NURE geochemical sampling. Very little prospecting. Mostly close to tidewater; most is steep and rugged. Moderate-size tract. $100 \%$ in USFS South Baranof Wilderness.

Discussion: 
Tract Name: Kake-Gunnuck and Sitkum Creeks.

Iract No: 01PE

Geology: Deformed and slightly metamorphosed Mesozoic and Paleozoic clastic and volcanic rocks may contain massive sulfide deposits.

Geochemistry; Bedrock anomalies: $\mathrm{Cu}, \mathrm{Zn}$ in north and west parts of tract; $\mathrm{Cu}, \mathrm{Pb}, \mathrm{Ni}, \mathrm{Cr}, \mathrm{Mo}$ in central and east parts. Stream-sediment anomalies: $\mathrm{Co}, \mathrm{Ni}$.

\section{Geophysics:}

Significant Deposits: None.

(w/Production and

Reserves)

\section{Undiscovered Deposit Type}

1) 28 a.1 Sierran kuroko massive sulfide

\section{Number of Undiscovered Deposits}

\begin{tabular}{|c|c|c|c|}
\hline 95 & 90 & 50 & 10 \\
\hline 0 & 0 & 0 & 0 \\
\hline
\end{tabular}

\section{Grade/Tonnage}

Model?

Yes

Tract $\left(\mathrm{km}^{2}\right): \quad 100$ total: 75 in PE and 25 in SD.

Comments: Reconnaissance geologic and geochemical mapping by USGS. Very little prospecting. Mostly close to tidewater; most is moderately steep; some is steep and rugged; local heavy timber and brush; logging roads. Moderate-size tract. $100 \%$ in Alaska Native lands.

Discussion: Includes USGS OFR 91-010 tracts 01PE and 08SD. 
Geology: Tertiary intermediate and mafic volcanic rocks cover Early Tertiary sandstone and conglomerate with possible U-Th resources.

Geochemistry: Bedrock anomalies: U.

Geophysics: Localized radiometric anomalies.

Significant Deposits: PE002, 003.

(w/Production and

Reserves)

\section{Undiscovered Deposit Type}

1) $30 \mathrm{c}$ Sandstone $U$

\section{Number of Undıscovered Deposits} at Probability Percentile

$\begin{array}{lllll}95 & 90 & 50 & 10 & 05\end{array}$

Grade/Tonnage

Model?

No

Tract $\left(\mathrm{km}^{2}\right): \quad 837$ total: 744 in PE and 93 in PA.

Comments: Reconnaissance and some semi-detailed geologic and geochemical mapping by USGS. Locally intense prospecting. Mostly close to tidewater; most is moderate relief. Some is steep and rugged; local heavy timber and brush; some logging roads. Large tract; $4 \%$ in USFS Tebenkof Bay Wilderness, $96 \%$ in USFS Tongass National Forest, some of which is proposed Research Natural Area.

Discussion: Includes USGS OFR 91-010 tracts 07PA and 03PE. 


\section{Southeastern Alaska}

Tract Name: Southwest Kupreanof

Iract No: 05PE

Geology: Middle Tertiary felsic and intermediate volcanic rocks occur in a possible eruptive center and in close association with granitic rocks of tract 06PE; some areas of intense alteration. Most of tract inferred to be underlain by Tertiary sandstone and conglomerate of the Kootznahoo Formation (see tract 03PE).

\section{Geochemistry:}

\section{Geophysics:}

Significant Deposits: PE004, 030.

(w/Production and

Reserves)

\section{Undiscovered Deposit Type}

1) $25 b$ Creede epithermal vein

2) 22c Polymetallic vein

3) $30 \mathrm{c}$ Sandstone U

\section{Number of Undiscovered Deposits} at Probability Percentile

$\begin{array}{lllll}95 & 90 & 50 & 10 & 05\end{array}$

$\begin{array}{lllll}0 & 0 & 0 & 1 & 2\end{array}$

$\begin{array}{lllll}0 & 0 & 0 & 1 & 2\end{array}$

0

0

0

\section{Grade/Tonnage}

Model?

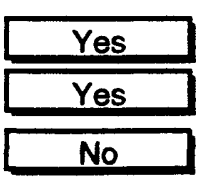

$\operatorname{Tract}\left(\mathrm{km}^{2}\right): \quad 640$

Comments: Reconnaissance and some detailed geologic and geochemical mapping by USGS; moderate amount of prospecting, including some drilling. Most is close to tidewater; local heavy timber and brush; large tract. $100 \%$ in USFS Tongass National Forest.

Discussion: U resources in deposit type 3) are included with those estimated for tract 03PE. 
Tract Name: (A): Tunehean Creek-Castle River, (B): Southeast Zarembo,

(C): Central Etolin, (D): Niblack and Deer Islands

Iract No: 06PE

Geology: Middle Tertiary alkalic and subalkalic granitic rocks intrude Cretaceous and other Mesozoic turbidites, other metasedimentary rocks, metavolcanic rocks, Cretaceous granitic rocks, and Tertiary sedimentary and volcanic rocks; closely associated with Tertiary volcanic rocks of tract 05PE to northwest, but more deeply eroded to the southeast.

Geochemistry: Stream-sediment anomalies: La, $\mathrm{Nb}, \mathrm{Y}, \mathrm{Pb}$.

Bedrock anomalies: $\mathrm{Be}, \mathrm{Nb}, \mathrm{Y}, \mathrm{Sn}, \mathrm{Cu}, \mathrm{Mo}, \mathrm{La}, \mathrm{Co}, \mathrm{Cr}$.

Geophysics: Strong, local aeroradioactivity anomalies.

Significant Deposits: None.

(w/Production and

Reserves)

\section{Undiscovered Deposit Type}

1) Felsic plutonic $U$

2) 11d Th-RE veins
Number of Undiscovered Deposits at Probability Percentile

$\begin{array}{lllll}95 & 90 & 50 & 10 & 05\end{array}$

$\begin{array}{lllll}0 & 0 & 0 & 0 & 1\end{array}$

$\begin{array}{lllll}0 & 0 & 0 & 0 & 1\end{array}$

\section{Grade/Tonnage}

Model?

(n)

1

Tract $\left(\mathrm{km}^{2}\right): \quad 330$ total: 28 in (A), 80 in (B), 213 in (C), and 9 in (D).

Comments: Reconnaissance and some detailed geologic and geochemical mapping by USGS; moderate amount of prospecting locally. Most is close to tidewater, some parts steep and rugged; local heavy timber and brush; some logging roads in (B) and (C). Large tract: $8 \%$ in USFS South Etolin Wildemess; $92 \%$ in USFS Tongass National Forest.

Discussion: 


\section{Southesstern Alaska}

Iract Name: Kosciusko-Northern Prince of Wales Islands

Iract No: 07PE

Geology: Contact metamorphosed homfels and marble of Paleozoic age in aureole of early Late Cretaceous granodiorite pluton are a classic skam environment.

Geochemistry: Stream-sediment anomalies: W, Mo.

Bedrock anomalies: Mo, $\mathrm{Cu}, \mathrm{Bi}$.

\section{Geophysics:}

Significant Deposits: PE005 (marble), 006-012. Reserves at PE007: 10,000 to 20,000 T w/ 1.5\% MoS2.

(w/Production and

Reserves)

\section{Undiscovered Deposit Type}

1) 21a Porphyry Cu-Mo

2) $18 \mathrm{~b}$ Cu skam

3) $22 \mathrm{c}$ Polymetallic vein
Number of Undiscovered Deposits at Probability Percentile

$95 \quad 90 \quad 50 \quad 10 \quad 05$

000001

$\begin{array}{llllll}0 & 0 & 0 & 1 & 2\end{array}$

$\begin{array}{lllll}0 & 0 & 1 & 2 & 3\end{array}$

\section{Grade/Tonnage}

Model?

\begin{tabular}{|c|}
\hline Yes \\
\hline Yes \\
\hline Yes \\
\hline
\end{tabular}

Tract $\left(\mathrm{km}^{2}\right): \quad 287$

Comments: Reconnaissance and some detailed geologic and geochemical mapping by USGS; moderate amount of prospecting locally. In part close to tidewater; some parts steep and rugged; local heavy timber and brush; some logging roads; moderate-size tract. $100 \%$ in USFS Tongass National Forest.

Discussion: 
Geology: Silurian turbidites and volcanic rocks are intruded by carbonatite and felsic dikes.

Geochemistry: Stream-sediment anomalies: Mo, La, Nb.

Bedrock anomalies: $\mathrm{Mo}, \mathrm{Zn}, \mathrm{Au}, \mathrm{Ag}, \mathrm{Cu}, \mathrm{Be}, \mathrm{La}, \mathrm{Nb}, \mathrm{Ba}$.

\section{Geophysics:}

Significant Deposits: PE013-015.

(w/Production and Reserves)

\section{Undiscovered Deposit Type}
1) 10 Carbonatite
2) Felsic plutonic $U$
3) 11d Th-RE veins

\section{Number of Undiscovered Deposits}

$\begin{array}{ccccc}\mathbf{5} & \text { at Probability Percentlle } & \\ \mathbf{9 5} & \mathbf{9 0} & \mathbf{5 0} & \mathbf{1 0} & \mathbf{0 5} \\ 0 & 0 & 0 & 0 & 1 \\ 0 & 0 & 0 & 0 & 1 \\ 0 & 0 & 0 & 0 & 1\end{array}$

Grade/Tonnage

Model?

\begin{tabular}{|c|}
\hline Yes \\
\hline No \\
\hline Yes \\
\hline
\end{tabular}

$\operatorname{Tract}\left(\mathrm{km}^{2}\right): \quad 16$

Comments: Reconnaissance geologic and geochemical mapping by USGS; moderate amount of prospecting locally. In part close to tidewater; heavy timber and brush; small tract. $100 \%$ in USFS Tongass National Forest.

Discussion: 
Geology: Alaskan-type mafic-ultramafic bodies of mid-Cretaceous age intrude Silurian turbidites and minor volcanic rocks at $(A)$ and Cretaceous turbidites at $(B)$.

Geochemistry: Stream-sediment anomalies: (A) Ni, $\mathrm{Cr}, \mathrm{Co}$; (B) $\mathrm{Co}, \mathrm{Ni}, \mathrm{Cu}$ Bedrock anomalies: (A) Ni, Cr, Cu, Co, $\mathrm{Pb}$; (B) $\mathrm{Ni}, \mathrm{Cr}, \mathrm{Cu}, \mathrm{Co}, \mathrm{Pb}, \mathrm{Zn}, \mathrm{Mo}$

Geophysics: Aeromagnetic anomalies: strong and steep-sided at (A) and (B).

\section{Significant Deposits: \\ (w/Production and \\ Reserves)}

(A): PE016.

(B): PE032.

Reserves: Large tonnage $w / 1$ to $2 \%$ sulfides.

\section{Undiscovered Deposit Type}

1) 9 Alaskan PGE
Number of Undiscovered Deposits

$$
\text { at Probabillty Percentile }
$$

$95 \quad 90 \quad 50 \quad 10 \quad 05$

0

$$
0
$$

0
Gradertonnage

Model?

No

Tract $\left(\mathrm{km}^{2}\right): \quad 34$ total: 10 in (A); 22 in PE and 2 in SD in (B).

Comments: Reconnaissance geologic and geochemical mapping by USGS; moderate amount of prospecting locally. (A) at tidewater, (B) close to tidewater. No relief at (A), moderate to steep at (B); local heavy timber and brush; small tract. $100 \%$ in USFS Tongass National Forest.

Discussion: Includes USGS OFR 91-010 tracts 10SD and 1OPE. 
Geology: Silurian turbidites and minor volcanic rocks are intruded by mid-Cretaceous granodionite.

Geochemistry: Stream-sediment anomalies: $\mathrm{Cr}, \mathrm{Ni}, \mathrm{Cu}$.

Bedrock anomalies: $\mathrm{Cu}, \mathrm{Pb}, \mathrm{Zn}, \mathrm{Cr}, \mathrm{Ni}, \mathrm{Co}$, in north part.

Geophysics:

Significant Deposits:

(w/Production and Reserves)

\section{Undiscovered Deposit Type}

1) 22c Polymetallic vein

\section{Number of Undiscovered Deposits}

\begin{tabular}{llll} 
at Probability Percentile & \\
\hline 5 & $90 \quad 50 \quad 10$ & 05
\end{tabular}

Grade/Tonnage

Model?

Yes

Tract $\left(\mathrm{km}^{2}\right): \quad 15$ total: 8 in $\mathrm{CR}$ and 7 in PE.

Comments: Reconnaissance geologic and geochemical mapping by USGS; minor amount of prospecting. Close to tidewater; moderate relief; local heavy timber and brush; some logging roads; small tract. $100 \%$ in USFS Tongass National Forest.

Discussion: Includes USGS OFR 91-010 tracts 04CR and 11PE. 


\section{Southeastern Alaska}

Tract Name: Duncan Canal-Zarembo Island

Iract No: 12PE

Geology: Deformed and slightly metamorphosed Mesozoic and Paleozoic clastic and volcanic rocks in Duncan Canal fault zone; large blocks of Devonian carbonate suggest that the major unit present before the youngest faulting was a melange; bedded barite and massive sulfide deposits present.

Geochemistry: Stream-sediment anomalies: Scattered W, Mo, Cu, Ba, Pb Bedrock anomalies: Strong $\mathrm{Ba}, \mathrm{Zn}, \mathrm{Pb}, \mathrm{Cu}$; some $\mathrm{Mo}, \mathrm{Cr}$, Co

\section{Geophysics:}

Significant Deposits: PE018-022, 027, 029, 031. Production from PE022: 750,000 T barite mined (w/Production and Reserves) 1965-1980. The other localities are included in an aggregated USBM estimate of $1,363,000 \mathrm{mt} w / 0.18 \mathrm{~g} / \mathrm{mt} \mathrm{Au}, 72.96 \mathrm{~g} / \mathrm{mt} \mathrm{Ag}, 0.04 \% \mathrm{Cu}, 0.34 \% \mathrm{~Pb}, 0.01 \% \mathrm{Zn}$ (Coldwell, 1990).

\section{Undiscovered Deposit Type}

1) $28 a .1$ Sierran kuroko massive sulfide

2) 31b Bedded barite

\section{Number of Undiscovered Deposits} at Probabillity Percentile

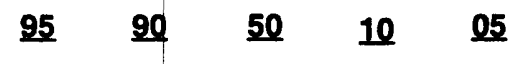

$\begin{array}{lllll}0 & 1 & 2 & 3 & 4\end{array}$

$\begin{array}{lllll}0 & 0 & 0 & 1 & 2\end{array}$
GraderTonnage Model?

\begin{tabular}{|c|}
\hline Yes \\
\hline Yes \\
\hline
\end{tabular}

Tract $\left(\mathrm{km}^{2}\right): \quad 722$ total: 717 in PE and 5 in SD.

Comments: Reconnaissance geologic and geochemical mapping by USGS; locally intensely prospected. Close to tidewater; moderate relief; local heavy timber and brush; some logging roads; large tract. $5 \%$ in Petersburg Creek- Duncan Salt Chuck Wilderness; $95 \%$ in USFS Tongass National Forest.

Discussion: Includes USGS OFR 91-010 tracts 09SD and 12PE. 
Geology: Mesozoic low-grade metavolcanic and minor metasedimentary rocks in the Duncan Canal fault zone; enigmatic deposit present.

Geochemistryi . Bedrock anomalies: $\mathrm{Zn}, \mathrm{Pb}, \mathrm{Cu}, \mathrm{Co}, \mathrm{Ag}$.

Geophysics:

Significant Deposits: PE017

(w/Production and

Reserves)

\section{Undiscovered Deposit Type}

1) 24a Cyprus massive sulfide(?)

2) $18 b$ Cu skarn(?)

\section{Number of Undiscovered Deposits} at Probability Percentlle

$\begin{array}{ccccc}95 & 90 & 50 & 10 & 05 \\ 0 & 0 & 0 & 0 & 1 \\ 0 & 0 & 0 & 0 & 1\end{array}$

Grade/Tonnage

Model?

\begin{tabular}{|c|}
\hline Yes \\
\hline Yes \\
\hline
\end{tabular}

Tract $\left(\mathrm{km}^{2}\right): 28$

Comments: Reconnaissance geologic and geochemical mapping by USGS; locally intensely prospected and drilled at PE017. Close to tidewater; moderate relief; local heavy timber and brush; small tract. 10\% in Petersburg Creek- Duncan Salt Chuck Wilderness; $90 \%$ in USFS Tongass National Forest.

\section{Discussion:}




\section{Southeastern Alaska}

Tract Name: Woewodski Island

Iract No: 14PE

Geology: Triassic and other Mesozoic low-grade metavolcanic and metasedimentary rocks in the Duncan Canal fault zone are intruded by a Cretaceous-age pluton; Au mines present.

Geochemistry: Stream-sediment anomalies: Au.

Bedrock Anomalies: $\mathrm{Au}, \mathrm{Ag}, \mathrm{Cu}, \mathrm{Pb}, \mathrm{Zn}, \mathrm{Co}, \mathrm{Cr}, \mathrm{Ni}, \mathrm{Mo}$.

Geophysics: Aeromagnetic anomaly: small and sharp-sided; may indicate a concealed pluton.

Significant Deposits: PE023-026. Production from PE025: more than $100 \mathrm{oz} \mathrm{Au}$ and $100 \mathrm{oz} \mathrm{Ag.}$

(w/Production and

Reserves)

\section{Undiscovered Deposit Type}

1) 36a Low-sulfide Au-quartz vein

2) 28a.1 Sierran kuroko massive sulfide
Number of Undiscovered Deposits at Probability Percentile

$\begin{array}{lllll}95 & 90 & 50 & 10 & 05\end{array}$

$\begin{array}{llllll}0 & 0 & 1 & 2 & 3\end{array}$

$\begin{array}{lllll}0 & 0 & 0 & 0 & 1\end{array}$

Grade/Tonnage Model?

Yes

Yes

Tract $\left(\mathrm{km}^{2}\right): \quad 30$

Comments: Reconnaissance geologic and geochemical mapping by USGS; locally intensely prospected and drilled at several localties. Close to tidewater; moderate relief; local heavy timber and brush; small, well explored tract. $100 \%$ in USFS Tongass National Forest.

Discussion: 
Geology: Mesozoic turbidites, other metasedimentary and metavolcanic rocks, and Cretaceous granitic rocks are intruded by Middle Tertiary alkalic and subalkalic rocks, producing vein and skam environments. Intrusive rocks elsewhere (in tract 05PE) have a close relation to the volcanic rocks.

Geochemistry: Stream-sediment anomalies: $\mathrm{Cr}, \mathrm{Ni}, \mathrm{Mo}, \mathrm{W}, \mathrm{Pb}, \mathrm{Nb}$. Bedrock anomalies: $\mathrm{Cu}, \mathrm{Pb}, \mathrm{Au}, \mathrm{Mo}, \mathrm{Zn}, \mathrm{Cr}, \mathrm{Ni}$.

Geophysics:

Significant Deposits: None.

(w/Production and

Reserves)

Undiscovered Deposit Type

1) $22 \mathrm{c}$ Polymetallic vein

2) $15 a \mathrm{~W}$ vein

\section{Number of Undiscovered Deposits} at Probability Percentile

$\begin{array}{lllll}95 & 90 & 50 & 10 & 05\end{array}$

$0 \quad 0 \quad 0 \quad 0 \quad 1$

$0 \quad 0 \quad 0 \quad 0 \quad 1$

Grade/Tonnage Model?

$\frac{\text { Yes }}{\text { Yes }}$

Tract $\left(\mathrm{km}^{2}\right): \quad 461$ total: 316 in PE, 25 in BC, 70 in CR, and 50 in KC.

Comments: Reconnaissance geologic and geochemical mapping by USGS; low level of prospecting. Close to tidewater; some parts steep and rugged; local heavy timber and brush; some logging roads; large tract. 20\% in USFS South Etolin wilderness; $80 \%$ in USFS Tongass National Forest.

Discussion: Includes USGS OFR 91-010 tracts 09BC, 01KC, 05CR, and 15PE. 
Geology: Fault cuts Cretaceous turbidites that were intruded by Late Cretaceous tonalite and Middle Tertiary granite.

Geochemistry: Bedrock anomalies: $\mathrm{Cu}, \mathrm{Mo}, \mathrm{Ni}$.

\section{Geophysics:}

Significant Deposits; None.

(w/Production and

Reserves)

\section{Undiscovered Deposit Type}

1) $22 \mathrm{c}$ Polymetallic vein

\section{Number of Undiscovered Deposits} at Probability Percentile

$95 \quad 90 \quad 50 \quad 10 \quad 05$

\section{Grade/Tonnage \\ Model?}

Yes

Tract $\left(\mathrm{km}^{2}\right): \quad 20$ total: 16 in PE, 4 in CR.

Comments: Reconnaissance geologic and geochemical mapping by USGS; low level of prospecting. Close to tidewater; local heavy timber and brush; some logging roads; small tract. $100 \%$ in USFS South Etolin Wilderness.

Discussion: Includes USGS OFR 91-010 tracts 06CR and 16PE. 
Tract Name: (A) Sukoi Islets, and (B) Northeast Mitkof Island Iract No: 17PE

Geology: Cretaceous turbidites and minor volcanic rocks are intruded locally by layered mid-Cretaceous (or Paleozoic?) hornblende gabbro and hormblendite plutons that contain magnetite.

Geochemistry: bedrock anomalies: $\mathrm{Cu}, \mathrm{Co}, \mathrm{Ni}, \mathrm{Cr}$.

Geophysics:

Significant Deposits: None.

(w/Production and

Reserves)

Undiscovered Deposit Type

1) 9 Alaskan PGE

\section{Number of Undiscovered Deposits} at Probability Percentile

$95 \quad 90 \quad 50 \quad 10 \quad 05$

Grade/Tonnage

Model?

No

Iract $\left(\mathrm{km}^{2}\right): \quad 12$

Comments: Reconnaissance geologic and geochemical mapping by USGS; low level of prospecting. At or close to tidewater; local heavy timber and brush; very small tract. $100 \%$ in USFS Tongass National Forest.

Discussion: 
Geology: Amphibolite and upper greenschist grade metapelitic, metacarbonate, and metavolcanic rocks of original mesozoic and Paleozoic age near the Great Tonalite Sill were intruded by evolved Late Tertiary $\mathrm{Sn}$-bearing granite and related rhyolite sills more or less along the Coast Range megalineament; stratiform Sn-base metal replacement deposits are present.

Geochemistry: Stream-sediment anomalies: Sn,Mo, W; strong.

Bedrock anomalies: $\mathrm{Sn}, \mathrm{Cu}, \mathrm{Pb}, \mathrm{Zn}, \mathrm{Mo}, \mathrm{Ni}, \mathrm{Cr}, \mathrm{Co}$.

\section{Geophysics:}

Significant Deposits: (w/Production and Reserves)

PE039, 040. Reserves at PE039: "several hundred thousand T" $w / 8.0 \% \mathrm{Zn}, 1.5 \%$ $\mathrm{Pb}, 1.5 \mathrm{oz} / \mathrm{T} \mathrm{Ag}$ in massive sulfide deposits" and "several hundred thousand $\mathrm{T"} \mathrm{W} /$ $2.5 \% \mathrm{Zn}, 1.0 \% \mathrm{~Pb}$ in disseminated deposits; at PE040: "many hundred thousand $\mathrm{T}^{n}$ $w / 1.6 \% \mathrm{Zn}, 0.1 \% \mathrm{~Pb}$ in disseminated deposits and "several million $\mathrm{T}^{\prime \prime} \mathrm{w} / 0.14 \% \mathrm{Zn}$, $0.09 \% \mathrm{~Pb}$ in quartz-fluorite vein deposits. These localities are included in a USBM aggregated inferred estimate of $9,830,000 \mathrm{mt} w / 2.14 \mathrm{~g} / \mathrm{mt} \mathrm{Ag}, 0.58 \% \mathrm{~Pb}, 1.78 \% \mathrm{Zn}$ (Coldwell, 1990).

\section{Undiscovered Deposit Type}

1) $14 \mathrm{c}$ Replacement $\mathrm{Sn}$

2) 28 a.1 Sierran kuroko massive sulfide

3) 36a Low-sulfide Au-quartz vein

\section{Number of Undiscovered Deposits} at Probability Percentile

$\begin{array}{lllll}95 & 90 & 50 & 10 & 05\end{array}$

\section{Grade/Tonnage} Model?

$\begin{array}{rrrr}0 & 1 & 2 & 3 \\ - & - & - & - \\ - & - & - & -\end{array}$

\begin{tabular}{|c|}
\hline Yes \\
\hline Yes \\
\hline Yes \\
\hline
\end{tabular}

Tract $\left(\mathrm{km}^{2}\right): \quad 43$ total: 36 in PE and 07 in BC.

Comments: Reconnaissance geologic and geochemical mapping by USGS; older USGS detailed mapping; moderate to high level of prospecting, with some deep drilling. Remote and steep; extensive snow and glacier cover; small tract. $100 \%$ in USFS Tongass National Forest.

Discussion: Includes USGS OFR 91-010 tracts 03BC and 2DPE. 
Geology: Ti-bearing magnetite occurs in Cretaceous zoned ultramafic body that intrudes Paleozoic metamorphic and Triassic gabbroic country rocks.

Geochemistry: Nonspecific geochem anomalies reported.

Geophysics: Aeromagnetic anomaly.

Significant Deposits: PR003-019.

(w/Production and

Reserves)

\section{Undiscovered Deposit Type}

1) 9 Alaskan PGE

\section{Number of Undiscovered Deposits} at Probabillity Percentile

$\begin{array}{lllll}95 & 90 & 50 & 10 & 05\end{array}$

- $\quad$ - 10.

Grade/Tonnage

Model?

No

Tract $\left(\mathrm{km}^{2}\right): \quad 165$ total: 162 in PR, 03 in KC.

Comments: Reconnaissance geologic and geochemical mapping by USGS; detailed geologic mapping by others; moderate to high level of prospecting. Close to tidewater; low relief; limited outcrop; moderate-size tract. $100 \%$ in USFS Tongass National Forest.

Discussion: Includes USGS OFR 91-010 tracts 17KC and 03PR. 
Geology: Au-bearing quartz veins occur in and close to mid-Cretaceous magnetite-rich pyroxenite and diorite.

\section{Geochemistry:}

Geophysics: Aeromagnetic anomaly: large and steep-sided.

Significant Deposits: SD003-005. Production from SD004 and 005: At least 2,000 oz Au mined in early . (w/Production and Reserves) 1900 's. Reserves for these two deposits are in a USBM aggregated inferred estimate of181,000 mT w/ 10.28 g/mt Au (Coldwell, 1990). Reserves for SD003: 500,00T w/ $18.9 \% \mathrm{Fe}, 2.6 \% \mathrm{Ti}, 0.7 \% \mathrm{~V}$; also a USBM inferred estimate of $454,000 \mathrm{mt} \mathrm{w} / 15.0 \%$ $\mathrm{Fe}, 0.09 \mathrm{~g} / \mathrm{mt} \mathrm{Pt}, 0.75 \% \mathrm{~V}$ (Coldwell, 1990).

\section{Undiscovered Deposit Type}

1) $36 a$ Low-sulfide Au-quartz vein

2) 9 Alaskan PGE

\section{Number of Undiscovered Deposits} at Probability Percentile

$\begin{array}{lllll}95 & 90 & 50 & 10 & 05\end{array}$

$\begin{array}{lllll}0 & 0 & 0 & 0 & 1\end{array}$

Grade/Tonnage Model?

No

Tract $\left(\mathrm{km}^{2}\right): \quad 23$

Comments: Reconnaissance geologic and geochemical mapping by USGS; detailed sampling by USBM; moderate to high level of prospecting and extensive drilling. In part close to tidewater; locally heavy timber; steep in part; small tract. $100 \%$ in USFS Tongass National Forest.

Discussion: 
Geology: Deformed and metamorphosed Paleozoic and Mesozoic clastic and volcanic rocks are intruded by latest Cretaceous Great Tonalite Sill; known sulfide deposits are in metamorphic rocks close to the sill and have been metamorphosed.

Geochemistry: Significant anomalies reported.

Geophysics: Aeromagnetic gradient: distinct, large, and steep.

Significant Deposits; SD006, 008, 009-011, 015-017, 021, 025, 036; PE 034. Production: From SD036: 50 (w/Production and oz Au in early 1900's. Reserves: At SD009: 7,300 T w/ $0.23 \mathrm{oz} / \mathrm{T} \mathrm{Au}, 0.31 \mathrm{oz} / \mathrm{T} \mathrm{Ag}$, Reserves) $0.7 \% \mathrm{Cu}$; at SD011: $187,000 \mathrm{~T}$ w/ 3.42\% Zn, $1.42 \% \mathrm{Cu}, 0.43 \mathrm{oz} / \mathrm{T} \mathrm{Ag}, 0.008 \mathrm{oz} / \mathrm{T}$ $\mathrm{Au}$; at SD016-017: 26,700,000 T w/ 0.57\% Cu, 0.37\% Zn, 0.3 oz/T Ag; all of these localities are included in a USBM inferred estimate of $25,041,000 \mathrm{mt} w / 0.01 \mathrm{~g} / \mathrm{mt}$ Au, $10.10 \mathrm{~g} / \mathrm{mt} \mathrm{Ag,} \mathrm{0.57 \%} \mathrm{Cu,} \mathrm{0.39 \%} \mathrm{Zn} \mathrm{(Coldwell,} \mathrm{1990).}$

\section{Undiscovered Deposit Type}

1) $28 a .1$ Sierran kuroko massive sulfide

2) 36a Low-sulfide Au-quartz vein

\section{Number of Undiscovered Deposits} at Probability Percentile

$\begin{array}{ccccc}95 & 90 & 50 & 10 & 05 \\ 0 & 0 & 1 & 2 & 4 \\ 0 & 0 & 0 & 0 & 1\end{array}$

Grade/Tonnage Model?

\begin{tabular}{|c|}
\hline Yes \\
\hline Yes \\
\hline
\end{tabular}

Tract $\left(\mathrm{km}^{2}\right): \quad 1,112$ total: 705 in SD and 407 in PE.

Comments: Reconnaissance geologic and geochemical mapping by USGS in north half of tract, less in south; moderate to high level of prospecting in north half and extensive drilling at SD009, 011, 016, 917. In part close to tidewater; locally heavy timber; in part remote and steep; extensive glacier and snow cover; very large tract. $38 \%$ in USFS Tracy Arm-Fords Terror Wilderness; $3 \%$ in USFS Chuck River Wilderness; $18 \%$ in USFS Stikine-LeConte Wilderness; $41 \%$ in USFS Tongass National Forest.

Discussion: Includes USGS OFR 91-010 tracts 18PE and 04SD. 
Geology: Au-quartz and polymetallic vein and possible metamorphosed Sierran-type massive sulfide deposits occur in highly deformed and variably metamorphosed Paleozoic and Mesozoic clastic, volcanic, and carbonate rocks that are intruded by Late Cretaceous granodiorite and tonalite plutons.

Geochemistry: Significant anomalies reported.

Geophysics:

Significant Deposits; SD019, 022-024, 026-035, 037-040. Production from SD022: 24,000 oz Au and (w/Production and Reserves) 24,000 oz Ag in early 1900's. Several Au-quartz veins in the Windham Bay area each produced a few thousand oz Au at about the same time, w/ average Au content of about $0.25 \mathrm{oz} / \mathrm{T}$. Reserves: Localities SD019, 021, and 025 are contained in a USBM aggregated inferred estimate of $390,000 \mathrm{mt} w / 4.69 \mathrm{~g} / \mathrm{mt} \mathrm{Au}, 7.10 \mathrm{~g} / \mathrm{mt} \mathrm{Ag}, 0.09 \%$ $\mathrm{Cu}, 0.28 \% \mathrm{~Pb}, 1.36 \% \mathrm{Zn}$; localities SD024, 026-027, 029-030, 034-035, 037, and probably $031-033$ are contained in a USBM aggregated inferred estimate of 164,000 $\mathrm{mt} w / 7.73 \mathrm{~g} / \mathrm{mt}$ Au.

\section{Number of Undiscovered Deposits}

\section{Undiscovered Deposit Type}

1) 28 a.1 Sierran kuroko massive sulfide

2) $36 \mathrm{a}$ Low-sulfide Au-quartz vein

\begin{tabular}{ccccc}
\multicolumn{5}{c}{ at Probability Percentile } \\
95 & $\mathbf{9 0}$ & $\mathbf{5 0}$ & 10 & 05 \\
0 & 0 & 0 & 1 & 2 \\
0 & 0 & 0 & 0 & 1
\end{tabular}

\section{Grade/tonnage \\ Model?}

2

1

Tract $\left(\mathrm{km}^{2}\right): \quad 537$

Comments: Reconnaissance geologic and geochemical mapping by USGS; moderate to high level of prospecting in early 1900's. In part close to tidewater; in part remote and steep; locally heavy timber; extensive glacier and snow cover; large tract. 50\% in USFS Chuck River Wilderness; $\mathbf{5 0} \%$ in USFS Tongass National Forest.

Discussion: 
Tract Name: Dawes Glacier-Buddington Range

Iract No: O6SD

Geology: Tertiary granodiorite crops out over a large area and contains several occurrences of $\mathrm{Cu}$ minerals in thin veinlets.

Geochemistry: Some anomalies reported.

Geophysics:

Significant Deposits: SD042, 043, 045, 046.

(w/Production and Reserves)

\section{Undiscovered Deposit Type}

1) 17.1 BC-AK Porphyry $\mathrm{Cu}$

2) 22c Polymetallic vein

Number of Undiscovered Deposits at Probability Percentile

$\begin{array}{ccccc}95 & 90 & 50 & 10 & 05 \\ 0 & 0 & 0 & 0 & 1 \\ 0 & 0 & 0 & 1 & 2\end{array}$

Grade/Tonnage

Model?

\begin{tabular}{|c|}
\hline Yes \\
\hline Yes \\
\hline
\end{tabular}

Tract $\left(\mathrm{km}^{2}\right): \quad 320$

Comments: Reconnaissance geologic and geochemical mapping by USGS; low level of prospecting. Remote; very rugged; extensive snow and ice cover; moderate-size tract tract. $5 \%$ in USFS Tracy Arm-Fords Terror Wilderness; $95 \%$ in USFS Tongass National Forest.

Discussion: 
Geology: Cretaceous magnetite-bearing gabbro intrudes Paleozoic and Mesozoic clastic and volcanic rocks; gabbro is interpreted to be the outer envelope of an Alaskan-type mafic-ultramafic body.

Geochemistry: Anomalies: $\mathrm{Co}, \mathrm{Cr}, \mathrm{Cu}, \mathrm{Mo}, \mathrm{Pb}, \mathrm{Zn}$.

Geophysics: Aeromagnetic anomaly: distinct, large, and steep-sided.

\section{Significant Deposits:}

(w/Production and

Reserves)

\section{Number of Undiscovered Deposits}

\section{Undiscovered Deposit Type}

\section{at Probability Percentile}

1) 9 Alaskan PGE

$\begin{array}{ccccc}95 & 90 & 50 & 10 & 05 \\ 0 & 0 & 0 & 0 & 1\end{array}$

\section{Grade/Tonnage}

Model?

No

$\operatorname{Tract}\left(\mathrm{km}^{2}\right): \quad 58$

Comments: Reconnaissance geologic and geochemical mapping by USGS; close to tidewater and logging roads; some heavy timber; small tract. $100 \%$ in Alaska Native lands.

Discussion: 
Geology: Cretaceous graywacke and Triassic(?) and Cretaceous greenstone are intrude by Tertiary gabbroic rocks that locally contain magmatic sulfide concentrations.

Geochemistry: Anomalies: $\mathrm{Ag}, \mathrm{As}, \mathrm{Au}, \mathrm{Co}, \mathrm{Cr}, \mathrm{Cu}, \mathrm{Ni}, \mathrm{W}, \mathrm{Zn}$.

Geophysics: Gravity anomaly: NW-trending high to W.

Significant Deposits: S1001, 002, 016-020, 021. Reserves: At S1002 (Bohemia Basin): 20,100,000 T w/ (w/Production and $0.31 \% \mathrm{Ni}, 0.18 \% \mathrm{Cu}, 0.04 \%$ Co; the USBM inferred estimate is $18,144,000 \mathrm{mt} \mathrm{w} /$ Reserves) $0.21 \% \mathrm{Cu}, 0.35 \% \mathrm{Ni}, 0,02 \% \mathrm{Co}$ (Coldwell, 1990 ); at Mirror Harbor: one deposit contains $8,000 \mathrm{~T} \mathrm{w} / 1.57 \% \mathrm{Ni}, 0.88 \% \mathrm{Cu}$; it has a USBM inferred estimate of $907,000 \mathrm{mt} \mathrm{w} / 0.13 \% \mathrm{Cu}, 0.33 \% \mathrm{Ni}$ (Coldwell, 1990); another deposit has several million $\mathrm{T} w / 0.2 \% \mathrm{Ni}, 0.1 \% \mathrm{Cu}$.

\section{Undiscovered Deposit Type}

1) $7 \mathrm{a}$ Synorogenic-synvolcanic $\mathrm{Ni}-\mathrm{Cu}$
Number of Undiscovered Deposits at Probability Percentile

$95 \quad 90 \quad 50 \quad 10 \quad 05$

$\begin{array}{lllll}0 & 0 & 0 & 1 & 2\end{array}$

Grade/Tonnage Model?

Tract $\left(\mathrm{km}^{2}\right): \quad 117$ total: 105 in SI and 12 in MF.

Comments: Detailed geologic and geochemical mapping by USGS; also geochemical sampling by USBM; intense local prospecting and drilling; close to tidewater; locally steep and rugged; some heavy timber; moderate-size tract. 50\% in USFS West Chichagof Wilderness; $50 \%$ in USFS Tongass National Forest.

Discussion: Includes USGS OFR 91-010 tracts 13MF and 01SI. 
Geology: Cretaceous graywacke and Triassic(?) and Cretaceous greenstone are intruded byTertiary granitic and gabbroic rocks (to north); local swarms of Au-bearing quartz veins parallel large linear shear zones; east side of tract includes greenstone, carbonate, and detrital clastic rocks that are in part older than other parts of tract.

Geochemistry: Anomalies associated with Cretaceous graywacke: $\mathrm{Ag}, \mathrm{As}, \mathrm{Au}, \mathrm{Cu}, \mathrm{Mo}, \mathrm{Pb}, \mathrm{W}, \mathrm{Zn}$. Anomalies associated with Triassic(?) and Cretaceous greenstones: $\mathrm{Ag}, \mathrm{Cu}, \mathrm{Pb}, \mathrm{Zn}$.

Geophysics: Gravity anomaly: NW-trending high to W.

Significant Deposits: S1006, 021, 023-050, 052, 054, 061-068; PA001-005. Production: From S1030 (w/Production and Reserves)

(Hirst-Chichagof): about 87,980 oz Au, 20,000 oz Ag; from S1036 (Chichagoff): about 700,000 oz Au, 200,000 oz Ag. Reserves: Chichagoff Mine indicated: 80,000 T w/ 0.025 oz/T Au, 0.08 oz/T Ag; inferred: $463,000 \mathrm{~T}$ w/ 0.3 oz $/ \mathrm{T} \mathrm{Au}, 0.09 \mathrm{oz} / \mathrm{T} \mathrm{Ag;}$ Chichagoff tailings indicated: $465,000 \mathrm{~T}$ w/ $0.11 \mathrm{oz} / \mathrm{T} \mathrm{Au}, 0.03 \mathrm{oz} / \mathrm{T} \mathrm{Ag}$; other inferred: $13,500 \mathrm{~T}$ w/ 0.11 oz/T Au, 0.04 oz/T Ag; $300,000 \mathrm{~T}$ w/ 0.04 oz $\mathrm{T} \mathrm{Au}, 0.012$ oz/T Ag; Hirst-Chichagof Mine inferred: $80,000 \mathrm{~T}$ w/ $1.0 \mathrm{oz} / \mathrm{T} \mathrm{Au}, 0.25 \mathrm{oz} / \mathrm{T} \mathrm{Ag;}$ $70,000 \mathrm{~T}$ w/ $0.25 \mathrm{oz} / \mathrm{T} \mathrm{Au}, 0.06 \mathrm{oz} / \mathrm{T} \mathrm{Ag}$; Hirst-Chichagof tailings inferred: 70,000 T w/ $0.14 \mathrm{oz} / \mathrm{Tu}, 0.03 \mathrm{oz} / \mathrm{T} \mathrm{Ag}$. Both of these localities are contained in a USBM aggregated inferred estimate of $1,173,000 \mathrm{mt} w / 17.12 \mathrm{~g} / \mathrm{m} \mathrm{Au}, 4.96 \mathrm{~g} / \mathrm{mt} \mathrm{Ag}$ (Coldwell, 1990).

\section{Undiscovered Deposit Type}

1) 36a Low-sulfide Au-quartz vein

2) 24a Cyprus massive sulfide

3) 23 Basaltic $\mathrm{Cu}$
Number of Undiscovered Deposits

\begin{tabular}{ccccc} 
& \multicolumn{4}{c}{ at Probability Percentile } \\
95 & 90 & 50 & 10 & 05 \\
0 & 0 & 1 & 2 & 3 \\
0 & 0 & 0 & 0 & 1 \\
0 & 0 & 0 & 0 & 1
\end{tabular}

Grade/Tonnage Model?

\begin{tabular}{|c|}
\hline Yes \\
\hline Yes \\
\hline Yes \\
\hline
\end{tabular}

Tract $\left(\mathrm{km}^{2}\right): \quad 836$ total: 680 in SI, 17 in MF, and 139 in PA.

Comments: Semi-detailed geologic and geochemical mapping by USGS; also geochemical sampling by USBM; intense local prospecting and drilling; close to tidewater, locally steep and rugged; some heavy timber. Large tract: about $50 \%$ in USFS West Chichagof Wilderness; $40 \%$ in USFS Tongass National Forest; $10 \%$ in Alaska State land, City and Borough of Sitka land, and private holdings.

Discussion: Includes USGS OFR 91-010 tracts 12MF, 02SI, and 02PA. 
Geology: Cretaceous and older greenstone, clastic, and carbonate rocks are intruded by locally foliated, generally sheared and altered, Jurassic and Cretaceous granitic rocks that contain Au-quartz veins.

Geochemistry: Anomalies: Ag, Au, As, Ba, $\mathrm{Cu}, \mathrm{Mo}, \mathrm{Pb}, \mathrm{W}, \mathrm{Zn}$ along major shear zone.

Geophysics: Gravity anomaly: NW-trending high to W.

Significant Deposits: SI003-005, 007--12, 015, MF020, 021. Production: SI 005 (Apex-EI Nido): >17,000 ( $w /$ Production and $0 \mathrm{z}$. Au, 2,400 oz Ag. Reserves: For same deposit: USBM has an inferred esimate of Reserves) $24,100 \mathrm{mt} \mathrm{w} / 32.91 \mathrm{~g} / \mathrm{mt} \mathrm{Au}$.

\section{Undiscovered Deposit Type}

1) 36a Low-sulfide Au-quartz vein

\section{Number of Undiscovered Deposits}

\begin{tabular}{ccccc} 
& \multicolumn{4}{c}{ at Probability Percentile } \\
95 & 90 & 50 & 10 & 05 \\
0 & 0 & 0 & 1 & 2
\end{tabular}

Model?

Yes

Tract $\left(\mathrm{km}^{2}\right): \quad 342$ total: 292 in SI and 50 in MF.

Comments: Semi-detailed geologic and geochemical mapping by USGS; also geochemical sampling by USBM; intense local prospecting and drilling; in part close to tidewater; locally steep and rugged; some heavy timber; moderate-size tract. $68 \%$ in USFS West Chichagof Wilderness; $32 \%$ in USFS Tongass National Forest.

Discussion: Includes USGS OFR 91-010 tracts 14MF and 03SI. 
Tract Name: Chichagof and Baranof Tertiary plutons: includes (A) Lake Elfindahl, (B) Rust Mountain, (C) Granite Islands, (D) Deep Bay, (E) Kruzof Island, (F) Takatz Bay, (G) Trap Bay, (H) Crawfish Bay-Gut Bay, and (I) Redfish Bay

Geology: Leucocratic Tertiary and Cretaceous(?) granodiorites intrude a variety of Cretaceous, older Mesozoic, and Paleozoic rocks; permissive for vein and porphyry deposits. (See tract 09PA.)

Geochemistry: Anomalies in (A), (B), (C), and (D): Ag, As, Au, Cu, Mo, Sn, W.

\section{Geophysics:}

Significant Deposits: (A) None; (B) None; (C) None; (D) None; (E) None; (F) S1076; (G) Slo82, 083; (H) (w/Production and PA014, 015; (I) PA018.

Reserves)

\section{Undiscovered Deposit Type}

1) 21a Porphyry Cu-Mo

2) 22c Polymetallic vein
Number of Undiscovered Deposits at Probability Percentile

$\begin{array}{lllll}95 & 90 & 50 & 10 & 05\end{array}$

\begin{tabular}{ll|lll}
0 & 0 & 0 & 0 & 1
\end{tabular}

$0 \quad 0 \quad 0 \quad 0 \quad 1$
Grade/Tonnage Model?

\begin{tabular}{|c|}
\hline Yes \\
\hline Yes \\
\hline
\end{tabular}

Tract $\left(\mathrm{km}^{2}\right): \quad 1,351$ total: see Discussion for breakdown.

Comments: Reconnaissance and some semi-detailed geologic and geochemical mapping by USGS; little prospecting; in part close to tidewater; locally steep and rugged; some heavy brush and timber; some glacier cover; very large tract. $6 \%$ in USFS West Chichagof Wildemess; $46 \%$ in South Baranof Wilderness; $47 \%$ in USFS Tongass National Forest.

Discussion; Includes the following USGS OFR 91-010 tracts: all of 04SI; sub-tract (F) includes 03(A)PA; sub-tracts $(H)$ and $(I)$ are $03(B) P A$ and $03(C) P A$, respectively. 
Geology: Upper Triassic(?) basalt flows and breccia are permissive for massive sulfide deposits.

Geochemistry: Anomalies: $\mathrm{Ag}, \mathrm{Cu}, \mathrm{Pb}, \mathrm{Zn} . \mathrm{Au}$.

Geophysics: Gravity anomaly: NW-trending linear high.

Significant Deposits: S1013, 014, 051, 053, 055, 056.

(w/Production and Reserves)

\section{Undiscovered Deposit Type}

1) 23 Basaltic $\mathrm{Cu}$
Number of Undiscovered Deposits

$$
\text { at Probability Percentile }
$$

$\begin{array}{lllll}95 & 90 & 50 & 10 & 05\end{array}$

$\begin{array}{lllll}0 & 0 & 0 & 1 & 2\end{array}$

Grade/Tonnage

Model?

No

Tract $\left(\mathrm{km}^{2}\right): \quad 238$

Comments: Reconnaissance and some semi-detailed geologic and geochemical mapping by USGS; little prospecting; relatively distant from tidewater, locally steep and rugged; some heavy brush and timber; moderate-size tract. 100\% in USFS West Chichagof Wilderness.

Discussion: 
Iract Name: (A) Tarn Mountain, and (B) Moore Mountains

Iract No: 07SI

Geology: Paleozoic carbonate layers in clastic rock section are hornfelsed by Cretaceous intrusions; defines a permissive skam environment.

Geochemistry:

Geophysics: Aeromagnetic anomaly: low in (A).

Significant Deposits: None.

(w/Production and Reserves)

\section{Undiscovered Deposit Type}

1) $18 \mathrm{c} \mathrm{Zn}-\mathrm{Pb}$ skam

2) $18 \mathrm{~b}$ Cu skam

3) 19a Polymetallic replacement

4) $22 c$ Polymetallic vein

\section{Number of Undiscovered Deposits}

\begin{tabular}{ccccc}
\multicolumn{5}{c}{ at Prohability Percentile } \\
$\mathbf{9 5}$ & $\mathbf{9 0}$ & $\mathbf{5 0}$ & $\mathbf{1 0}$ & $\mathbf{0 5}$ \\
$\mathbf{0}$ & 0 & 0 & 0 & 1 \\
0 & 0 & 0 & 0 & 1 \\
0 & 0 & 0 & 0 & 1 \\
0 & 0 & 0 & 0 & 1
\end{tabular}

Model?

\begin{tabular}{|c|}
\hline Yes \\
\hline Yes \\
\hline Yes \\
\hline Yes \\
\hline
\end{tabular}

Tract $\left(\mathrm{km}^{2}\right): \quad 77$ total: (A) 28 in SI and 09 in MF; (B) 40.

Comments: Reconnaissance geologic mapping by USGS; little prospecting; in part close to tidewater; locally steep and rugged; some heavy brush and timber; small tract. $100 \%$ in USFS Tongass National Forest.

Discussion: Includes USGS OFR 91-010 tracts 07SI and 15MF. 
Tract Name: Tenakee Inlet, South of head of

Iract No: $08 \mathrm{SI}$

Geology: Tertiary(?) leucogabbros intrude Paleozoic clastic rocks; gabbros may be related to the magmatic-sulfide-bearing gabbros in tract $01 \mathrm{SI}$.

\section{Geochemistry:}

Geophysics:

Significant Deposits: None.

(w/Production and

Reserves)

Undiscovered Deposit Type

1) 7 a Synorogenic-synvolcanic $\mathrm{Ni}-\mathrm{Cu}$
Number of Undiscovered Deposits

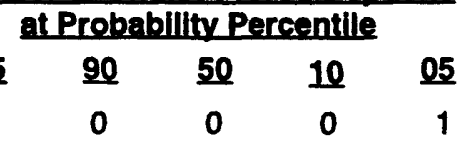

Grade/Tonnage

Model?

Yes

Tract $\left(\mathrm{km}^{2}\right): 61$

Comments: Reconnaissance geologic mapping by USGS; little prospecting; in part close to tidewater; locally steep and rugged; some heavy brush and timber; small tract. $100 \%$ in USFS Tongass National Forest.

Discussion: 
Geology: Paleozoic carbonate rocks are intruded and hornfelsed by Cretaceous quartz monzonite pluton; Mississippian section may contain evaporite deposits or contact-metamorphic gypsum.

Geochemistry:

Geophysics:

Significant Deposits; S1085-087. Production: From S1086 and 087: 500,000 T gypsum (Flint and Cobb, (w/Production and 1952). Reserves)

\section{Undiscovered Deposit Type}

1) $18 b$ Cu skam

2) $18 \mathrm{c} \mathrm{Zn-Pb} \mathrm{skarn}$

3) Gypsum

\section{Number of Undiscovered Deposits} at Probability Percentile

$\begin{array}{lllll}95 & 90 & 50 & 10 & 05\end{array}$

$\begin{array}{lllll}0 & 0 & 0 & 0 & 1\end{array}$

$\begin{array}{lllll}0 & 0 & 0 & 0 & 1\end{array}$

$\begin{array}{lllll}0 & 0 & 0 & 0 & 1\end{array}$

\section{Grade/tonnage}

Model?

\begin{tabular}{|c|}
\hline Yes \\
\hline Yes \\
\hline No \\
\hline
\end{tabular}

Tract $\left(\mathrm{km}^{2}\right): \quad 123$ total: 93 in SI and 30 in JU.

Comments: Some detailed, but mostly reconnaissance geologic and geochemical mapping by USGS; little prospecting; in part close to tidewater; locally steep and rugged; some heavy brush and timber. $100 \%$ in USFS Tongass National Forest.

Discussion: Includes USGS OFR 91-010 tracts 10SI and 08JU. 
Tract Name: Tenakee-Sitkoh Bay

Iract No: $11 \mathrm{SI}$

Geology: Silurian and Jurassic syenitic rock suites intrude Paleozoic clastic and carbonate rocks; alkalic rocks may host vein deposits of $U, T h$, and REE.

Geochemistry:

Geophysics:

Significant Deposits: S1078.

(w/Production and

Reserves)

Undiscovered Deposit Type

1) 11d Th-REE veins
Number of Undiscovered Deposits

at Probability Percentile

$\begin{array}{lllll}95 & 90 & 50 & 10 & 05\end{array}$

$0 \quad 0 \quad 0 \quad 0 \quad 1$

\section{Grade/Tonnage}

Model?

Yes

Tract $\left(\mathrm{km}^{2}\right): \quad 187$

Comments: Reconnaissance geologic mapping by USGS; some private and USBM prospecting; in part close to tidewater; locally steep and rugged; some heavy brush and timber. $100 \%$ in USFS Tongass National Forest.

Discussion: 
Geology: Non-manine sandstone, shale, conglomerate, and coal of the Tertiary Kootznahoo Formation underlie a limited portion of the tract.

Geochemistry: High U/Th ratio for most samples.

Geophysics:

Significant Deposits: SI104; other small coal mines and prospects. Production: Small amount of coal for (w/Production and Reserves)

\section{Undiscovered Deposit Type}

1) $30 \mathrm{c}$ Sandstone $U$

2) Coal

\section{Number of Undiscovered Deposits}

\begin{tabular}{ccccc}
\multicolumn{5}{c}{ at Probability Percentile } \\
95 & 90 & 50 & 10 & 05 \\
0 & 0 & 0 & 0 & 1 \\
- & - & - & - & -
\end{tabular}

Grade/Tonnage

Model?

No

\section{Tract $\left(\mathrm{km}^{2}\right): 67$}

Comments: Semi-detailed and reconnaissance geologic and geochemical mapping for $U$ and Th by USGS; some private prospecting; close to tidewater; low relief; some heavy brush and timber; relatively small tract. $100 \%$ in USFS Admiralty Island Monument and Kootznoowoo Wilderness; but about $20 \%$ of the above is also covered by Alaska Native village lands.

Discussion: 
Geology: Highly deformed and locally metamorphosed Late Triassic mafic and intermediate volcanic rocks, fine-grained clastic rocks, and ultramafic masses host significant massive sulfide, $\mathrm{Ni}$-Cu magmatic segregation, and polymetallic vein deposits to the north in Tract 09JU.

Geochemistry: Stream-sediment anomalies: abundant and varied.

\section{Geophysics:}

Significant Deposits: S1093, 094, 097, 100-103, 105-112.

(w/Production and Reserves)

\section{Undiscovered Deposit Type}

1) 28a.1 Sierran kuroko massive sulfide

2) 22c Polymetallic vein
Number of Undiscovered Deposits at Probability Percentile

$\begin{array}{ccccc}95 & 90 & 50 & 10 & 05 \\ 0 & 0 & 0 & 0 & 1 \\ 0 & 0 & 0 & 0 & 1\end{array}$
Model?

\begin{tabular}{|c|}
\hline Yes \\
\hline Yes \\
\hline
\end{tabular}

Tract $\left(\mathrm{km}^{2}\right): \quad 1,339$ total: 1,327 in SI and 12 in SD.

Comments: Reconnaissance and some semi-detailed geologic and geochemical mapping by USGS; some private prospecting in past; close to tidewater in part; most is steep and rugged; some heavy brush and timber; relatively large tract. About $95 \%$ in USFS Admiralty Island Monument and Kootznoowoo Wilderness; $5 \%$ in Alaska Native lands.

Discussion; Includes USGS OFR 91-010 tracts 13SI and 12SD. Tract 09JU is geologic continuation to north. 
Tract Name: King Salmon Bay

Iract No: $14 \mathrm{SI}$

Geology: Slightly metamorphosed Late(?) Triassic intermediate and mafic volcanic rocks are permissive for massive sulfide deposits.

Geochemistry:

Geophysics:

Significant Deposits: S1092.

(w/Production and

Reserves)

\section{Undiscovered Deposit Type}

1) $28 a .1$ Sierran kuroko massive sulfide
Number of Undiscovered Deposits at Probabillity Percentile

$95 \quad 90 \quad 50 \quad 10 \quad 05$

$\begin{array}{lllll}0 & 0 & 0 & 0 & 1\end{array}$

Grade/Tonnage

Model?

Yes

Tract $\left(\mathrm{km}^{2}\right): \quad 30$ total: 26 in Sl and 04 in JU.

Comments: Reconnaissance geologic and geochemical mapping by USGS; some private prospecting in past; close to tidewater, some heavy brush and timber, small tract. $100 \%$ in USFS Admiralty Island Monument and Kootznoowoo Wilderness.

Discussion: Includes USGS FR 91-010 tracts 14SI and 10JU. 
Geology: Discontinuous carbonate lenses in Silurian and (or) Devonian clastic rocks are intruded by Cretaceous granitic rocks and skam deposits are present.

Geochemistry. Bedrock anomalies: float below suggests that more deposits may be present.

\section{Geophysics:}

Significant Deposits: SK004. Reserves: At SK004: 4,300 T inferred w/ 0.5\% W, 5.0\% Cu, 7.0 oz/T Ag, (w/Production and Reserves) 0.15 oz/T Au; or 3,900 mt inferred w/ $5.14 \mathrm{~g} / \mathrm{mt} \mathrm{Au}, 239.97 \mathrm{~g} / \mathrm{mt} \mathrm{Ag,} 0.5 \% \mathrm{Cu}, 0.66$ $\mathrm{g} / \mathrm{mt}$ WO3 (Coldwell, 1990).

\section{Undiscovered Deposit Type}

1) $14 a$ W skam

2) $18 \mathrm{~b}$ Cu skam

3) $18 \mathrm{c} \mathrm{Zn-Pb} \mathrm{skam}$

\section{Number of Undiscovered Deposits}

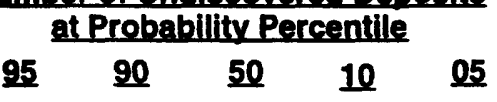

\section{GraderTonnage Model?}

$\begin{array}{ccccc}- & - & - & - & - \\ - & - & - & - & - \\ - & - & - & - & -\end{array}$

Yes

Yes

Yes

Tract $\left(\mathrm{km}^{2}\right): \quad 36$

Comments: Reconnaissance geologic and geochemical mapping by USGS and USBM; intense prospecting by USBM; moderately remote; steep and rugged; very small tract. $100 \%$ in USNPS Glacier Bay National Park.

Discussion: Tract is too small for probabilistic quantitative estimate. 
Tract Name: Takhinsha Mountains

Iract No: $07 \mathrm{SK}$

Geology: Paleozoic clastic and carbonate rocks are intruded and metamorphosed by Cretaceous and Tertiary granodiorite dikes and plutons.

Geochemistry: Bedrock anomalies: $\mathrm{Ag}, \mathrm{Au}, \mathrm{Cu}, \mathrm{Zn}$.

\section{Geophysics:}

Significant Deposits: SK007, 008, 010, 011. (w/Production and Reserves)

\section{Undiscovered Deposit Type}

1) 21a Porphyry Cu-Mo
Number of Undiscovered Deposits at Probability Percentile

$95 \quad 90 \quad 50 \quad 10 \quad 05$

$\begin{array}{lllll}0 & 0 & 0 & 0 & 1\end{array}$

\section{Grade/Tonnage}

Model?

Yes

Tract $\left(\mathrm{km}^{2}\right): \quad 799$

Comments: Reconnaissance geologic and geochemical mapping by USGS and ADGGS; remote; steep and rugged; large tract. $65 \%$ in USNPS Glacier Bay National Park; $35 \%$ in Alaska State lands.

Discussion: 


\section{Southeastern Alaska}

Tract Name: Mount Henry Clay

Tract No: 09SK

Geology: Paleozoic and Mesozoic(?) volcanic and fine-grained clastic rocks are intruded by Tertiary granitic plutons; Ba-bearing massive sulfides present; also one locality with abundant large massive sulfide boulders.

Geochemistry: Stream-sediment and bedrock anomalies present.

\section{Geophysics:}

Significant Deposits: SK017-025. Reserves: At SK021: USBM inferred estimate of 750,000 T w/ $1.8 \mathrm{oz} / \mathrm{T}$ (w/Production and $\mathrm{Ag}, 1.7 \% \mathrm{Zn}, 60.0 \% \mathrm{Brt}$; or $680,000 \mathrm{mt}$ inferred $\mathrm{w} / 60 \mathrm{~g} / \mathrm{mt} \mathrm{Ag,} 1.73 \mathrm{~g} / \mathrm{mt} \mathrm{Zn,} 60.0 \%$ Reserves) Brt (Coldwell, 1990).

\section{Undiscovered Deposit Type}

1) $28 a .1$ Sierran kuroko massive sulfide

2) $36 a$ Low-sulfide Au-quartz vein
Number of Undiscovered Deposits at Probability Percentile

$95 \quad 90 \quad 50 \quad 10 \quad 05$

$\begin{array}{lllll}0 & 0 & 1 & 2 & 3\end{array}$

$0 \quad 0 \quad 0 \quad 1 \quad 2$

Grade/Tonnage Model?

\begin{tabular}{|c|}
\hline Yes \\
\hline Yes \\
\hline
\end{tabular}

$\operatorname{Tract}\left(\mathrm{km}^{2}\right): 84$

Comments: Reconnaissance and detailed geologic and geochemical mapping by USGS, USBM, and ADGGS; moderate to high level of prospecting and some drilling; close to major road and trails; steep and rugged; extensive glacier cover; small tract. $100 \%$ in Alaska State lands.

Discussion: 


\section{Southeastern Alaaka}

Tract Name: (A) Surgeon Mountain, and (B) upper Tsirku River

Iract No: $10 \mathrm{SK}$

Geology: Paleozoic clastic, carbonate, and volcanic rocks intruded by Tertiary plutons; several small skarn deposits in Canada close to (A); some stratiform disseminated and massive sulfides in (B).

Geochemistry:

Geophysics:

Significant Deposits: None in (A) or (B).

(w/Production and

Reserves)

\section{Undiscovered Deposit Type}

1) 28 a.1 Sierran kuroko massive sulfide

2) $36 \mathrm{a}$ Low-sulfide Au-quartz vein

3) 18b Cu skarn
Number of Undiscovered Deposits at Probability Percentile

$\begin{array}{lllll}95 & 90 & 50 & 10 & 05\end{array}$

$\begin{array}{lllll}0 & 0 & 0 & 0 & 1\end{array}$

$\begin{array}{lllll}0 & 0 & 0 & 0 & 1\end{array}$

$\begin{array}{lllll}0 & 0 & 0 & 1 & 2\end{array}$

\section{Grade/Tonnage}

Model?

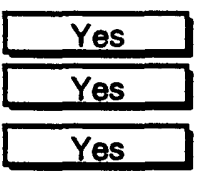

Tract $\left(\mathrm{km}^{2}\right): \quad 98$

Comments: Reconnaissance and detailed geologic and geochemical mapping by USGS, USBM, and ADGGS; moderate level of prospecting; close to major road and trails; steep and rugged; some glacier cover; small tract. $100 \%$ in Alaska State lands.

Discussion: 


\section{Southeastern Alaska}

Tract Name: (A) Porcupine, (B) lower Tsirku River, and (C) Takhin River

Iract No: $115 K$

Geology: Paleozoic fine-grained clastic, carbonate, and volcanic rocks are intruded by Cretaceous and Tertiary granitic plutons.

Geochemistry: Stream-sediment and bedrock anomalies present.

\section{Geophysics:}

Significant Deposits: (A): SK026-039, 051, 052; (B): SK009, 040-045, 047, 048; (C): SK050. Production: (w/Production and Reserves)

(A) Placer in early 1900's. Reserves: (A): For the area centered at SKO35 aggregated inferred estimates: Dredge: $3,396,000 \mathrm{mt} \mathrm{w} / 0.22 \mathrm{~g} / \mathrm{mt} \mathrm{Au}$; Alluvial: $308,600 \mathrm{mt} \mathrm{w} / 0.73 \mathrm{~g} / \mathrm{mt} \mathrm{Au}$.

\section{Undiscovered Deposit Type}

1) 36a Low-sulfide Au-quartz vein

2) 22c Polymetallic vein

3) 39a Placer Au-PGE

\section{Number of Undiscovered Deposits at Probability Percentile}

$\begin{array}{lllll}95 & 90 & 50 & 10 & 05\end{array}$

$\begin{array}{lllll}0 & 0 & 0 & 0 & 1\end{array}$

$\begin{array}{lllll}0 & 0 & 0 & 0 & 1\end{array}$

$\begin{array}{lllll}0 & 0 & 0 & 0 & 1\end{array}$

Grade/Tonnage

Model?

\begin{tabular}{|c|}
\hline Yes \\
\hline Yes \\
\hline Yes \\
\hline
\end{tabular}

Tract $\left(\mathrm{km}^{2}\right): \quad 498$

Comments: Reconnaissance and detailed geologic and geochemical mapping by USBM and ADGGS; moderate to high level of private prospecting; close to major road and trails; moderately steep and rugged; some glacier cover; large tract. $100 \%$ in Alaska State lands.

Discussion: 
Geology: Paleozoic fine-grained clastic, carbonate, and volcanic rocks, some of which were metamorphosed in Late Paleozoic, are intruded by Cretaceous and Tertiary plutons; sulfide-bearing quartz veins, altered zones, and some stratiform massive and disseminated sulfides are present.

Geochemistry: Bedrock anomalies: $\mathrm{Ag}, \mathrm{Ba}, \mathrm{Co}, \mathrm{Cu}, \mathrm{Zn}$.

\section{Geophysics:}

Significant Deposits; SK015, 046, 053-064.

(w/Production and

Reserves)

\section{Undiscovered Deposit Type}

1) 28 a.1 Sierran kuroko massive sultide

2) 36a Low-sulfide Au-quartz vein

3) 22c Polymetallic vein

\section{Number of Undiscovered Deposits}

\section{at Probability Percentile}

$\begin{array}{ccccc}95 & 90 & 50 & 10 & 05 \\ 0 & 0 & 0 & 0 & 1 \\ 0 & 0 & 0 & 0 & 1 \\ 0 & 0 & 0 & 0 & 1\end{array}$

Grade/Tonnage

Model?

\begin{tabular}{|c|}
\hline Yes \\
\hline Yes \\
\hline Yes \\
\hline
\end{tabular}

Iract $\left(\mathrm{km}^{2}\right): \quad 423$

Comments: Reconnaissance and detailed geologic and geochemical mapping by USBM and ADGGS; moderate to high level of private prospecting; close to major road, trails, and tidewater; moderately steep and rugged; some glacier cover; large tract. $100 \%$ in Alaska State lands.

Discussion: 
Geology: Upper Triassic metabasalts are intruded by magnetite-rich ultramafic body; fan below consists of broken rock from that body.

\section{Geochemistry:}

Geophysics: An aeromagnetic anomaly must exist with this large ultramafic body.

Significant Deposits: SK066, 067. Reserves: At SK066: lode: USBM estimate of 3,500,000,000 T inferred

(w/Production and Reserves) $w / 16.8 \mathrm{Fe}, 2.0 \% \mathrm{Ti}$, and $50,000,000 \mathrm{~T} w / 0.03 \mathrm{oz} / \mathrm{T} \mathrm{Au}, 0.1 \% \mathrm{Cu}, 0.03 \mathrm{oz} / \mathrm{T} \mathrm{Pt}, 0.03$ 0z/T Pd; at SK067: fan: 980,000,000 T interred w/ 10.8\% Fe, 2.0\% Ti. Also for the lode: a USBM estimate of 3,175,148,000 mt inferred $w / 16.8 \% \mathrm{Fe} ; 45,359,000 \mathrm{mt}$ inferred $w / 0.01 \mathrm{~g} / \mathrm{mt} \mathrm{Au}, 0.10 \% \mathrm{Cu}, 0.01 \mathrm{~g} / \mathrm{mt} \mathrm{Pt}, 0.01 \mathrm{~g} / \mathrm{mt} \mathrm{Pd}$; and for the fan, $898,789,600 \mathrm{mt} \mathrm{w} / 10.8 \% \mathrm{Fe}$ (Coldwell, 1990).

\section{Undiscovered Deposit Type}

1) 9 Alaskan PGE

\section{Number of Undiscovered Deposits} at Probability Percentile

$\begin{array}{lllll}95 & 90 & 50 & 10 & 05\end{array}$

\section{Grade/Tonnage Model?}

$\operatorname{Tract}\left(\mathrm{km}^{2}\right): 21$

Comments: Reconnaissance and detailed geologic and geochemical mapping by USGS, USBM and ADGGS; moderate to high level of private prospecting; close to major road and tidewater; in part very steep and rugged, but fan is easily accessible; small tract. About $5 \%$ in Alaska Native lands; $95 \%$ in Alaska State lands.

Discussion: 
Tract Name; (A) Chilkat River, and (B) east side of Chilkat Inlet,

Geology: Upper Triassic basalts are locally magnetite- and sulfide-bearing.

Geochemistry: Anomalies reported.

\section{Geophysics:}

Significant Deposits: (A): SK071-073; (B): SK078-081. Reserves: At (B): USBM indicated estimateof 700 T (w/Production and Reserves) w/ $0.09 \mathrm{oz} / \mathrm{T} \mathrm{Au}, 0.17 \mathrm{oz} / \mathrm{T} \mathrm{g}, 0.8 \% \mathrm{Cu}$; also USBM inferred estimate: $2,722,200$ $\mathrm{mt} \mathrm{w} / 0.27 \mathrm{~g} / \mathrm{mt} \mathrm{Au}$ (Coldwell, 1990).

\section{Undiscovered Deposit Type}
1) 23 Basaltic $\mathrm{Cu}$
2) 6a Low-sulfide Au-quartz vein

\section{Number of Undiscovered Deposits}

\section{at Probability Percentile}

$\begin{array}{ccccc}95 & 90 & 50 & 10 & 05 \\ 0 & 0 & 0 & 0 & 1 \\ 0 & 0 & 0 & 0 & 1\end{array}$

\section{Grade/Tonnage} Model?

$\operatorname{Tract}\left(\mathrm{km}^{2}\right): \quad 80$

Comments: Reconnaissance and detailed geologic and geochemical mapping by USGS, USBM and ADGGS; prospecting and drilling by USBM; moderate to high level of private prospecting; close to major road and tidewater; $(A)$ is in part steep and rugged, but $(B)$ is low relief; relatively small tract. $100 \%$ in Alaska State lands, including a State Park.

\section{Discussion:}


Geology: Upper Triassic metabasalts are intruded by a magnetite-rich ultramafic body.

Geochemistry: Some anomalies reported.

Geophysics: Gravity and aeromagnetic anomalies: large.

Significant Deposits: SK075-077. Reserves: At SK077: USBM estimate of "several million T" inferred w/ (w/Production and $<10 \%$ magnetite, about $1.0 \% \mathrm{Ti}$. Reserves)

\section{Number of Undiscovered Deposits}

Undiscovered Deposit Type

1) 9 Alaskan PGE

at Probability Percentile
$\quad 90 \quad 50 \quad 10 \quad 05$

Grade/Tonnage

Model?

No

$\operatorname{Tract}\left(\mathrm{km}^{2}\right): 26$

Comments: Some detailed geologic and geochemical mapping by USGS, USBM and ADGGS; minor private prospecting; close to major road and tidewater; low relief; relatively small tract under the town of Haines. $100 \%$ in Alaska State lands, including a State Park.

Discussion: 
Geology: Paleozoic and older(?) metamorphosed clastic, carbonate, and volcanic rocks are intruded by mid-Tertiary plutons.

Geochemistry: NURE stream-sediment anomalies: $\mathrm{Pb}$.

Geophysics:

Significant Deposits: SK082, 084-086. reserves: At SK084: USBM estimate of 10,000 T inferred w/ 0.04 (w/Production and Reserves) oz/T Au, 3.4\% Pb, 2.3\% $\mathrm{Zn}$; also an estimate of $9,000 \mathrm{mt} w / 13.71 \mathrm{~g} / \mathrm{mt} \mathrm{Au}, 3.4 \%$ $\mathrm{Pb}, 2.3 \% \mathrm{Zn}$ (Coldwell, 1990).

\section{Number of Undiscovered Deposits}

\section{Undiscovered Deposit Type}

1) 14a W skam

2) $18 b$ Cu skarn

3) $18 \mathrm{c} \mathrm{Zn-Pb} \mathrm{skarn}$

4) 21a Porphyry Cu-Mo

\section{at. Probability Percentile}

$95 \quad 90 \quad 50 \quad 10 \quad 05$

\begin{tabular}{ll|lll}
- & - & - & - & - \\
- & - & - & - & - \\
0 & 0 & 0 & 0 & 1 \\
0 & 0 & 0 & 0 & 1
\end{tabular}

\section{Grade/Tonnage} Model?

Tract $\left(\mathrm{km}^{2}\right): \quad 547$

Comments: Some detailed geologic and some geochemical mapping by USGS, USBM and ADGGS; prospecting by USBM; close to railroad, major road, and tidewater; near the town of Skagway. Fairly steep. Relatively large tract: $60 \%$ in Alaska State lands; $40 \%$ in USFS Tongass National Forest-- and parts of both in Trail of ' 98 NPS National Historical Park.

Discussion: 
Geology: Paleozoic and older metamorphosed clastic, carbonate, and volcanic rocks are intruded by mid-Tertiary plutons.

\section{Geochemistry:}

\section{Geophysics:}

Significant Deposits: None.

(w/Production and Reserves)

\section{Undiscovered Deposit Type}

1) $14 a W$ skam

2) $18 b$ Cu skam

3) $18 \mathrm{c} \mathrm{Zn-Pb} \mathrm{skam}$

\section{Number of Undiscovered Deposits} at Probabillity Percentile

$95 \quad 90 \quad 50 \quad 10 \quad 05$

$\begin{array}{ccccc}- & - & - & - & - \\ - & - & - & - & - \\ 0 & 0 & 0 & 0 & 1\end{array}$

\section{Grade/Tonnage}

Model?

\begin{tabular}{|c|}
\hline Yes \\
\hline Yes \\
\hline Yes \\
\hline
\end{tabular}

Tract $\left(\mathrm{km}^{2}\right): \quad 135$ total: 83 in SK and 52 in $\mathrm{AL}$.

Comments: Reconnaissance geologic and some geochemical mapping by USGS; essentially unprospected; remote, rugged, extensive glacier cover; relatively small tract. $100 \%$ in USFS Tongass National Forest.

Discussion: Includes USGS OFR 91-010 tracts 17SK and 01(A)AL. 
Geology: (A): Tertiary granodiorite or granite contains minor occurrences of disseminated motybdenite and some molybdenite-bearing quartz veins. (B): Late Tejiary myolite dike swarm associated with felsic plug intrudes metamorphosed Paleozoic and older(?) clastic, carbonate, and volcanic rocks.

\section{Geochemistry:}

Geophysics:

Significant Deposits: (A): TR001, 002; (B): TR004. Reserves: At TR002: USBM estimate of 907,000 mt w/ (w/Production and Reserves) $0.10 \%$ Mo (Coldwell, 1990).

Number of Undiscovered Deposits

Undiscovered Deposit Type

1) $21 \mathrm{~b}$ Porphyry Mo (low F)

2) $18 b$ Cu skarn

3) $18 \mathrm{c} \mathrm{Zn-Pb} \mathrm{skarn}$

\begin{tabular}{ccccr}
\multicolumn{5}{c}{ at Probability Percentile } \\
$\mathbf{9 5}$ & $\mathbf{9 0}$ & $\mathbf{5 0}$ & $\mathbf{1 0}$ & $\mathbf{0 5}$ \\
0 & 0 & 0 & 0 & 1 \\
0 & 0 & 0 & 0 & 1 \\
0 & 0 & 0 & 0 & 1
\end{tabular}

Grade/Tonnage Model?
Yes

Yes

Tract $\left(\mathrm{km}^{2}\right): \quad 225$

Comments: Reconnaissance geologic and some geochemical mapping by USGS; both parts essentially unprospected in US, but $(B)$ is well prospected across the Boundary in Canada; remote, rugged, extensive glacier cover; moderate-size tract, but tract (A) is small. $100 \%$ in USFS Tongass National Forest.

\section{Discussion:}


Geology: Complexly deformed and metamorphosed Paleozoic and older(?) clastic, volcanic, and minor carbonate rocks with scattered ultramafic masses are intruded by Tertiary granodiorite and granite; ultramafic masses probably contain chromite.

\section{Geochemistry:}

\section{Geophysics:}

Significant Deposits: TR003.

(w/Production and

Reserves)

Undiscovered Deposit Type

1) 8 a Podiform chromite (minor)

2) 22c Polymetallic vein

\section{Number of Undiscovered Deposits} at Probability Percentile

$95 \quad 90 \quad 50 \quad 10 \quad 05$

$\begin{array}{lllll}0 & 0 & 0 & 0 & 1\end{array}$

$0 \quad 0 \quad 0 \quad 0 \quad 1$

\section{Grade/Tonnage}

Model?

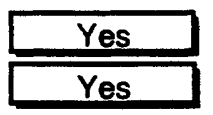

Tract $\left(\mathrm{km}^{2}\right): \quad 156$

Comments: Reconnaissance geologic and geochemical mapping by USGS; essentially unprospected; remote, rugged, extensive glacier cover; close to major river valley; relatively small tract. $100 \%$ in USFS Tongass National Forest (except for some Alaska State land and private homesites along river).

\section{Discussion:}


Geology: Paleozoic and older metamorphosed clastic, volcanic, and carbonate rocks are intruded by Tertiry plutons; an environment permissive for skarn deposits.

\section{Geochemistry:}

\section{Geophysics:}

Significant Deposits: TR005, 009.

(w/Production and

Reserves)

\section{Undiscovered Deposit Type}

\section{Number of Undiscovered Deposits} at Probability Percentile
1) $18 b$ Cu skam
2) $18 \mathrm{c} \mathrm{Zn-Pb} \mathrm{skarn}$
3) 22c Polymetallic vein

95

90

50

10

05

$0 \quad 0 \quad 0 \quad 0 \quad 1$

0

0

0

0

1

0

0

0

0

Grade/tonnage

Model?

\begin{tabular}{|c|}
\hline Yes \\
\hline Yes \\
\hline Yes \\
\hline
\end{tabular}

Tract $\left(\mathrm{km}^{2}\right): \quad 536$ total: 436 in TR and 100 in SD.

Comments: Reconnaissance geologic and geochemical mapping by USGS; little prospected; remote, rugged, very extensive glacier cover, relatively large tract. $59 \%$ in USFS Tracy Arm-Fords Terror Wilderness; $41 \%$ in USFS Tongass National Forest.

Discussion: Includes USGS OFR 91-010 tracts 03SD and 04TR. 
Geology: Altered zones and disseminated sulfides occur in Mesozoic and older(?) metamorphic rocks intruded by Jurassic, Cretaceous, and Tertiary granitic plutons.

Geochemistry: Anomalies: Mo, Ag, Au, Cu, Zn.

Geophysics:

Significant Deposits: YA009-011.

(w/Production and

Reserves)

\section{Undiscovered Deposit Type}

1) 28a.1 Sierran kuroko massive sulfide

2) 36 a Low-sulfide Au-quartz vein

3) 21a Porphyry Cu-Mo

\section{Number of Undiscovered Deposits}

$\begin{array}{ccccc}\mathbf{5} & \text { at Probability Percentile } & \\ 0 & \mathbf{9 0} & \mathbf{5 0} & \mathbf{1 0} & \mathbf{0 5} \\ 0 & 0 & 0 & 0 & 1 \\ 0 & 0 & 0 & 0 & 1 \\ 0 & 0 & 0 & 0 & 1\end{array}$

Grade/Tonnage

Model?

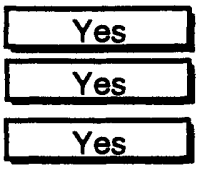

Tract $\left(\mathrm{km}^{2}\right): \quad 3,008$ total: 1,802, in YA, 948 in SK, 88 in ME, and 170 in MF.

Comments: Reconnaissance geologic and geochemical mapping by USGS; essentially unprospected; remote, rugged, very extensive glacier cover; extrmely large tract. $52 \%$ in USNPS Glacier Bay National Park; $9 \%$ in USFS Russell Fiord Wildemess; $3 \%$ in USNPS Wrangell-Saint Elias National Park and Preserve; $36 \%$ in USFS Tongass National Forest.

Discussion: Includes USGS OFR 91-010 tracts 01YA, 01SK, 01MF, and newly designated tract 01MEin nd. Sriat ielias quadraugle to northwest. 


\section{Southeastern Alaska}

Tract Name: Yakutat Range

Iract No: 02YA

Geology: Thin Au-bearing quartz veins occur in greenschist or lower-grade flyschoid rocks; veins are spatially and genetically related to Tertiary plutons.

\section{Geochemistry:}

\section{Geophysics:}

Significant Deposits: YA008.

(w/Production and

Reserves)

\section{Number of Undiscovered Deposits \\ at Probability Percentile}

\section{Undiscovered Deposit Type}

1) $36 a$ Low-sulfide Au-quartz vein

$95 \quad 90 \quad 50 \quad 10 \quad 05$

$\begin{array}{lllll}0 & 0 & 1 & 3 & 5\end{array}$

\section{Grade/Tonnage}

Model?

Yes

Tract $\left(\mathrm{km}^{2}\right): \quad 3,603$ total: 2,768 in YA, 598 in ME, 214 in MF, and 23 in SK.

Comments: Reconnaissance geologic and geochemical mapping by USGS; essentially unprospected; remote, rugged, extensive glacier cover; extremely large tract. $14 \%$ in USNPS Glacier Bay National Park; $36 \%$ in USFS Russell Fiord Wilderness; $4 \%$ in USNPS Glacier Bay National Preserve; $22 \%$ in USNPS Wrangell-Saint Elias National Park and Preserve; $24 \%$ in USFS Tongass National Forest.

Discussion: Includes USGS OFR 91-010 tracts 02YA, 02SK, 02MF, and newly designated tract 02MEın mat. Sraut Elixs guad to not thwest. 
Geology: Iron- and titanium-bearing beach and (locally) stream placers border the Gulf of Alaska; they include both modern beaches and older upraised marine terrace placers; iron- and titanium-bearing black sands $7 c c u r$ in lenses up to $3-\mathrm{m}$ thick of limited lateral extent.

\section{Geochemistry:}

\section{Geophysics:}

Significant Deposits: YA001-007, 012, 013; MF002, 011, 019. Production: In YA: During early 1900's (w/Production and Reserves) about $6 \mathrm{~kg}$ Au was produced from small deposits; in MF: about 4,000 oz Au produced from sands between 1890 and 1917 by small-scale operations. Reserves: In YA: Large, low-grade $\mathrm{Fe}$ and Ti tonnages present w/ an estimated $20.8 \mathrm{~kg} / \mathrm{m3} \mathrm{Fe}$, $12.2 \mathrm{~kg} / \mathrm{m} 3 \mathrm{Ti}$; some higher grade zones present; in MF: USBM inferred estimates calculated for 12 blocks covering a total area of 2.6 square $\mathrm{km}$ (two areas within those blocks contain resources $w /$ higher grades than present overall): $4,600,000$ $\mathrm{m3} \mathrm{w} / 1.0 \%$ ilmenite, minor Au (for 1977 value of $\$ 1.11 / \mathrm{m3}$; this includes (a) $153,000 \mathrm{m3} w / 3.4 \%$ ilmenite at one locality $w / 1977$ value of $\$ 3.83 / \mathrm{m3}$, and (b) $102,000 \mathrm{m3} \mathrm{w} / 4.2 \%$ ilmenite $\mathrm{w} / 1977$ value of $\$ 5.22 / \mathrm{m} 3$.

\section{Undiscovered Deposit Type}

1) $39 \mathrm{c}$ Shoreline placer $\mathrm{Ti}$

\section{Number of Undiscovered Deposits}

$\begin{array}{ccccc}95 & 90 & 50 & 10 & 05 \\ 0 & 1 & 2 & 3 & 4\end{array}$

Grade/Tonnage Model?

Tract $\left(\mathrm{km}^{2}\right): \quad 894$ total: 767 in YA and 27 in MF.

Comments: In YA: Reconnaissance and some detailed sampling by USBM, including auger-hole sampling; small amount of prospecting; local aeromagnetic survey by USGS; in MF reconnaissance and some detailed sampling by USBM; moderate amount of prospecting. Modern, bare beaches are easy to sample and explore, dense vegetation hinders exploration of back-beachdeposits, which are poorly known. Very large tract. $31 \%$ in USNPS Glacier Bay National Park; 9\% in USFS Russell Fiord Wilderness; $4 \%$ in USNPS Glacier Bay National Preserve; $17 \%$ in USFS Tongass National Forest.

Discussion: Includes USGS OFR 91-010 tracts 03YA and 03MF. 